Article

\title{
Design, Synthesis and Biological Evaluation of Isoxazole-Based CK1 Inhibitors Modified with Chiral Pyrrolidine Scaffolds
}

\author{
Andreas Luxenburger ${ }^{1, *,+}$, Dorian Schmidt ${ }^{2}$, Chiara Ianes ${ }^{3}$, Christian Pichlo ${ }^{4}{ }^{\oplus}$, Marc Krüger ${ }^{3}$, \\ Thorsten von Drathen ${ }^{2}$, Elena Brunstein ${ }^{4}$, Graeme J. Gainsford ${ }^{1}$, Ulrich Baumann ${ }^{4}$, \\ Uwe Knippschild ${ }^{3}$ and Christian Peifer ${ }^{2, *,+}$ \\ 1 Ferrier Research Institute, Victoria University of Wellington, 69 Gracefield Rd, Lower Hutt 5040, \\ New Zealand; gjgainsford@gmail.com \\ 2 Institute of Pharmacy, Christian-Albrechts-University of Kiel, Gutenbergstraße 76, D-24116 Kiel, Germany; \\ dschmidt@pharmazie.uni-kiel.de (D.S.); T.vonDrathen@gmx.de (T.v.D.) \\ 3 Department of General and Visceral Surgery, Ulm University Hospital, Albert-Einstein-Allee 23, \\ D-89081 Ulm, Germany; chiara.ianes@uni-ulm.de (C.I.); marcm3012@googlemail.com (M.K.); \\ uwe.knippschild@uniklinik-ulm.de (U.K.) \\ 4 Institute of Biochemistry, University of Cologne, Zuelpicher Str. 47, D-50674 Cologne, Germany; \\ pichloc@uni-koeln.de (C.P.); Elena.Brunstein@uni-koeln.de (E.B.); ubaumann@uni-koeln.de (U.B.) \\ * Correspondence: Andreas.Luxenburger@vuw.ac.nz (A.L.); cpeifer@pharmazie.uni-kiel.de (C.P.); \\ Tel.: +64-4-4630055 (A.L.); +49-431-880-1137 (C.P.) \\ + These two authors contribute equally to this work.
}

Academic Editor: Derek J. McPhee

Received: 12 December 2018; Accepted: 27 February 2019; Published: 1 March 2019

\begin{abstract}
In this study, we report on the modification of a 3,4-diaryl-isoxazole-based CK1 inhibitor with chiral pyrrolidine scaffolds to develop potent and selective CK1 inhibitors. The pharmacophore of the lead structure was extended towards the ribose pocket of the adenosine triphosphate (ATP) binding site driven by structure-based drug design. For an upscale compatible multigram synthesis of the functionalized pyrrolidine scaffolds, we used a chiral pool synthetic route starting from methionine. Biological evaluation of key compounds in kinase and cellular assays revealed significant effects of the scaffolds towards activity and selectivity, however, the absolute configuration of the chiral moieties only exhibited a limited effect on inhibitory activity. X-ray crystallographic analysis of ligand-CK1 $1 \delta$ complexes confirmed the expected binding mode of the 3,4-diaryl-isoxazole inhibitors. Surprisingly, the original compounds underwent spontaneous Pictet-Spengler cyclization with traces of formaldehyde during the co-crystallization process to form highly potent new ligands. Our data suggests chiral "ribose-like" pyrrolidine scaffolds have interesting potential for modifications of pharmacologically active compounds.
\end{abstract}

Keywords: protein kinase CK1; formerly known as casein kinase 1; chiral kinase inhibitors; iminoribitol; ribose pocket; 3,4-diaryl-isoxazole; Pictet-Spengler cyclization

\section{Introduction}

To date, many drug discovery programs have been based on commercially or readily available building blocks and reagents to enable rapid synthesis of large compound libraries destined for various screening campaigns [1]. This approach often resulted in collections of large, "flat", achiral and low-diverse molecules [2,3]. In this context, the "quality" of compounds and building blocks in terms of complexity, carbon bond saturation, chirality or natural product-likeness has been intensively discussed in the medicinal chemistry community [4]. 
In the field of protein kinases, most small molecule kinase inhibitors (SMKI) target the adenosine triphosphate (ATP) binding site in the catalytic cleft of the kinase (Figure 1A). Depending on their particular binding mode, several different types of inhibitors have been categorized [5]. Typical ATP-competitive kinase inhibitors (type I inhibitors) generally consist of a central nitrogen-containing heterocycle that is appended with mainly hydrophobic, rigid and achiral side chains. This design originates from Traxler's pharmacophore model that defines distinct subsites within the ATP binding pocket, which can be sampled to develop selective inhibitors (Figure 1B) [6].

A

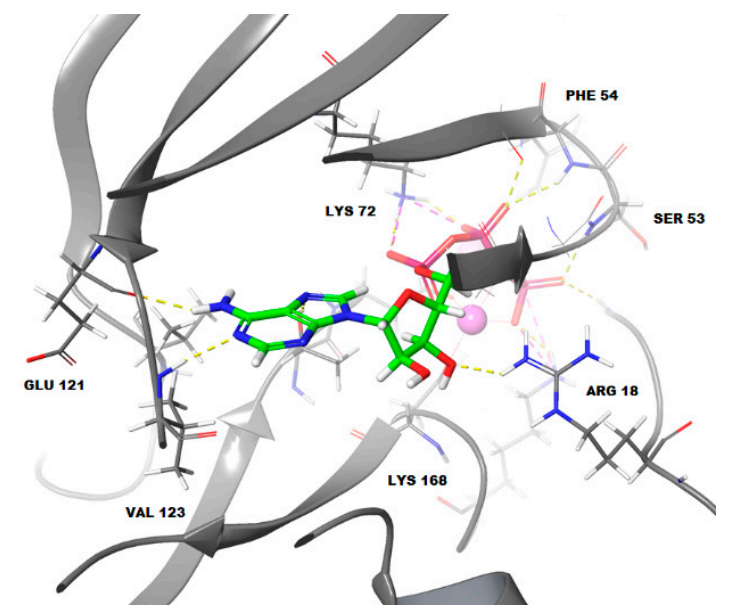

B

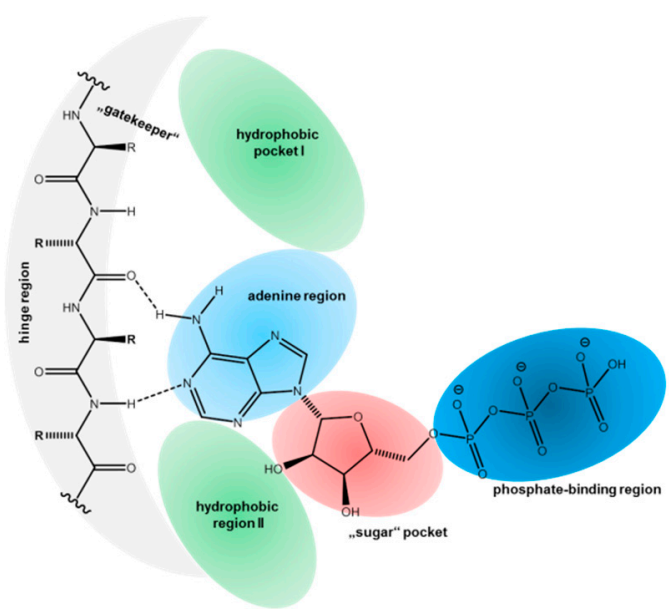

Figure 1. (A) Binding mode of adenosine triphosphate (ATP) within the binding pocket of cyclic adenosine monophosphate (cAMP) dependent protein kinase (pdb 4WB5 [7]). Hydrogen-bond interactions are shown as dashed lines. (B) Schematic representation of binding areas within the ATP binding site showing adapted interaction areas as suggested by the Traxler model.

Usually the central heterocycle binds to the "adenine region" of the ATP binding site and undergoes hydrogen bonding to the so-called hinge region of the kinase, whereas the appended side chains of the heterocycle facilitate binding interactions within the "hydrophobic pocket I" and the solvent-exposed "hydrophobic region II" $[5,6]$. This approach inevitably gives rise to lipophilic, "flat" compounds that are often found to come with poor pharmakokinetic ADME parameters at the later stages of drug development [8].

In contrast, the more polar regions of the ATP binding site (ribose pocket and phosphate-binding region) have been exploited less frequently. This may be because binding to these hydrophilic water-exposed areas does not necessarily translate into an increased binding enthalpy of an inhibitor. Therefore, these regions are often only used to implement water-solubilizing groups (e.g., functionalized tetrahydropyran (1), pyrrolidine (2) or piperidine moieties) that enhance pharmacokinetic parameters, such as oral bioavailability (Figure 2) [9-13]. However, residues that comprise the ribose pocket have also been successfully targeted through stereochemically defined interactions, to create highly potent and selective inhibitors [14-18]. Examples include Pfizer's interleukin-1 receptor-associated kinase 4 (IRAK4) inhibitor PF06650833 (3) [18], which is equipped with a chiral pyrrolidone scaffold, Merck's carboribose-functionalized diaminopyrimidine derivative 4 [17] and a series of p38 mitogen-activated protein kinase (MAPK) inhibitors (e.g., 5) developed by Laufer and co-workers [16]. A special case is the well-known microbial alkaloid staurosporine (6) that targets the ribose pocket with a chiral "glycosyl" subunit (cf. Figure 2). This moiety is reported to contribute to the high potency, but also to the very high kinase promiscuity of staurosporine (6) [19-23]. Interestingly, modification of the sugar-like moiety of several closely related indolocarbazole analogs resulted in enhanced protein kinase selectivity profiles (e.g., 7) [24-26]. Taken together, these examples illustrate the potential of a stereochemical approach to developing potent and selective kinase inhibitors $[19,27,28]$. 
<smiles>CN1CCN(CCOc2cc(OCC3CCOCC3)c3c(Nc4c(Cl)ccc5c4OCO5)ncnc3c2)CC1</smiles>

1<smiles>COc1cc2nc(-c3cc(F)ccc3O)nc(N[C@@H]3CNC[C@@H]3C(C)(C)O)c2cc1OC</smiles>

2

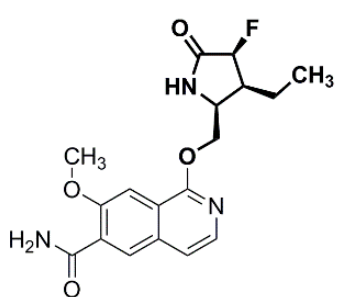

3<smiles>Cc1cc(Nc2ncc(-c3nc4ccncc4s3)c(NC3CC(CO)[C@@H](O)[C@H]3O)n2)cc(C)n1</smiles>

4<smiles>CC(C)C(C)Nc1cc(-c2[nH]c(SCC(O)CO)nc2-c2ccc(F)cc2)ccn1</smiles>

5

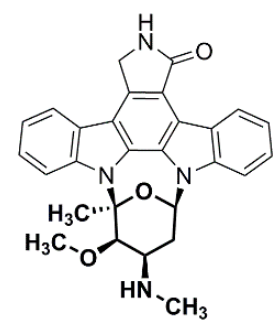

6

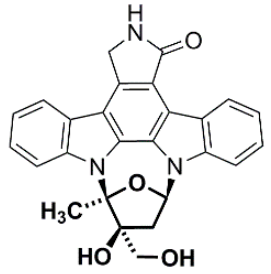

7

Figure 2. Selected kinase inhibitors targeting the ribose pocket or phosphate binding region with chiral hydrophilic moieties. 1: $\mathrm{IC}_{50}(\mathrm{c}-\mathrm{Src})=2.7 \mathrm{nM}$ [11]; 2: $\mathrm{IC}_{50}(\mathrm{CHK} 2)=3 \mathrm{nM}$ [12]; 3: $\mathrm{IC}_{50}(\mathrm{IRAK} 4)=0.2$ $\mathrm{nM}$ [18]; 4: $\mathrm{IC}_{50}(\mathrm{IRAK} 4)=0.3 \mathrm{nM}$ [17]; 5: $\mathrm{IC}_{50}(\mathrm{p} 38 \alpha)=15 \mathrm{nM}$ [16]; staurosporine (6): Non-selective kinase inhibitor; 7 : $\mathrm{IC}_{50}($ Trk $)=25 \mathrm{nM}, \mathrm{IC}_{50}(\mathrm{FLT3})=2-3 \mathrm{nM}, \mathrm{IC}_{50}(\mathrm{JAK} 2)=0.9 \mathrm{nM}$ [19].

In 2009, our group reported isoxazole 8 (Scheme 1) as a nanomolar inhibitor of the protein kinase $\mathrm{CK} 1 \delta\left[\mathrm{IC}_{50}(\mathrm{CK} 1 \delta)=0.033 \mu \mathrm{M}\right]$ [29]. CK1 $\delta$ is a Ser/Thr-specific protein kinase and is one of seven mammalian CK1 isoforms $(\alpha, \beta, \gamma 1, \gamma 2, \gamma 3, \delta$ and $\varepsilon)$ within the CK1 family. All CK1 isoforms share a highly conserved kinase domain [30] and phosphorylate a high number of substrates, including the tumor suppressor protein p53 [30,31]. Based on the wide spectrum of substrate proteins CK1 family members play an important role in the diverse signaling pathways that are involved in cell division, apoptosis, membrane transport, immune response and inflammation, spindle and centrosome-associated processes, DNA damage-related signal transduction and circadian rhythm. Deregulation and dysfunction of CK1 isoforms are associated with proliferative disorders, such as cancer [32]. Moreover, mutations, as well as disorders of CK1 expression and activity, have been observed in neurodegenerative diseases, such as Alzheimer's or Parkinson's disease, as well as sleeping disorders [32,33].
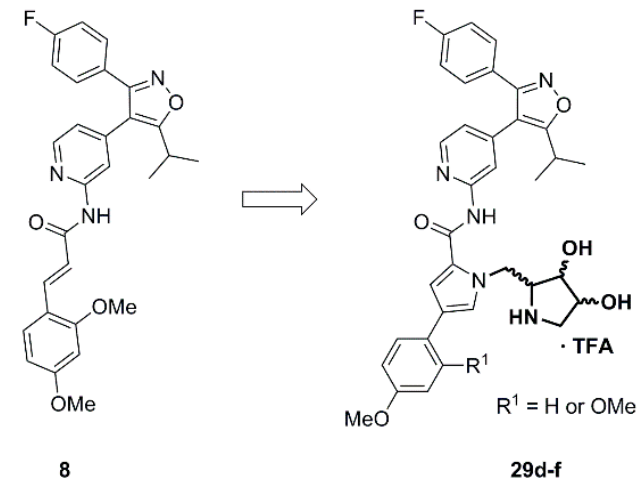

29d-f

Scheme 1. Inhibitor design concept. Based on protein kinase CK1 $\delta$ inhibitor 8 the cinnamic acid side chain was modified to attach chiral pyrrolidine scaffolds. 
In the present study, we have modified diaryl-isoxazole 8 with functionalized enantiopure pyrrolidine scaffolds (Scheme 1) to promote selective binding interactions in the more hydrophilic areas of the ATP binding pocket. These kinds of pyrrolidines scaffolds have been frequently used in various medicinal chemistry projects, including the development of glycosidase inhibitors, azanucleosides and antiviral agents [34-38]. Especially hydroxy-functionalized pyrrolidines are remarkable scaffolds, as they can be regarded as sugar analogues with the furanose ring oxygen substituted by a nitrogen atom. Due to their structural and chemical similarity these "iminosugars" are effective carbohydrate mimics and are ideal scaffolds to address carbohydrate-related targets, including nucleoside or nucleotide-binding enzymes. In line with this notion, "iminosugars" provide access to polar active sites and thus to a chemical space that is usually inaccessible to Lipinski-compliant molecules [34].

\section{Results and Discussion}

\subsection{Molecular Modelling}

To understand how the lead compound 8 could be elaborated to exploit residues in the ribose pocket, compound 8 was docked into a CK1 $1 \delta$-PF670462 co-crystal structure (pdb: 3UZP). According to the predicted binding mode the amidopyridinyl moiety occupies the adenine region of the ATP binding site and forms a bidentate interaction with the backbone NH and carbonyl oxygen of Leu-85 (Figure 3). The isoxazole ring is predicted to pack between the sidechains of Ile-23 and Ile-148, and to form a hydrogen bond between the ring nitrogen atom and a structural water. The $p$-fluorophenyl substituent packs tightly in the hydrophobic pocket I, formed between the sidechains of Lys-38, Met- 80 and the gate keeper residue Met-82. This characteristic "teardrop"-shaped [39] binding mode agrees with other related 4,5-diarylimidazoles that have been crystallized with CK1 Regarding isoxazole $\mathbf{8}$ this pharmacophore is extended towards the solvent-exposed hydrophobic region II by a cinnamic acid moiety. This configuration is projecting the aryl system to the solvent exposed area but leaving space for further interactions within the actual binding pocket.
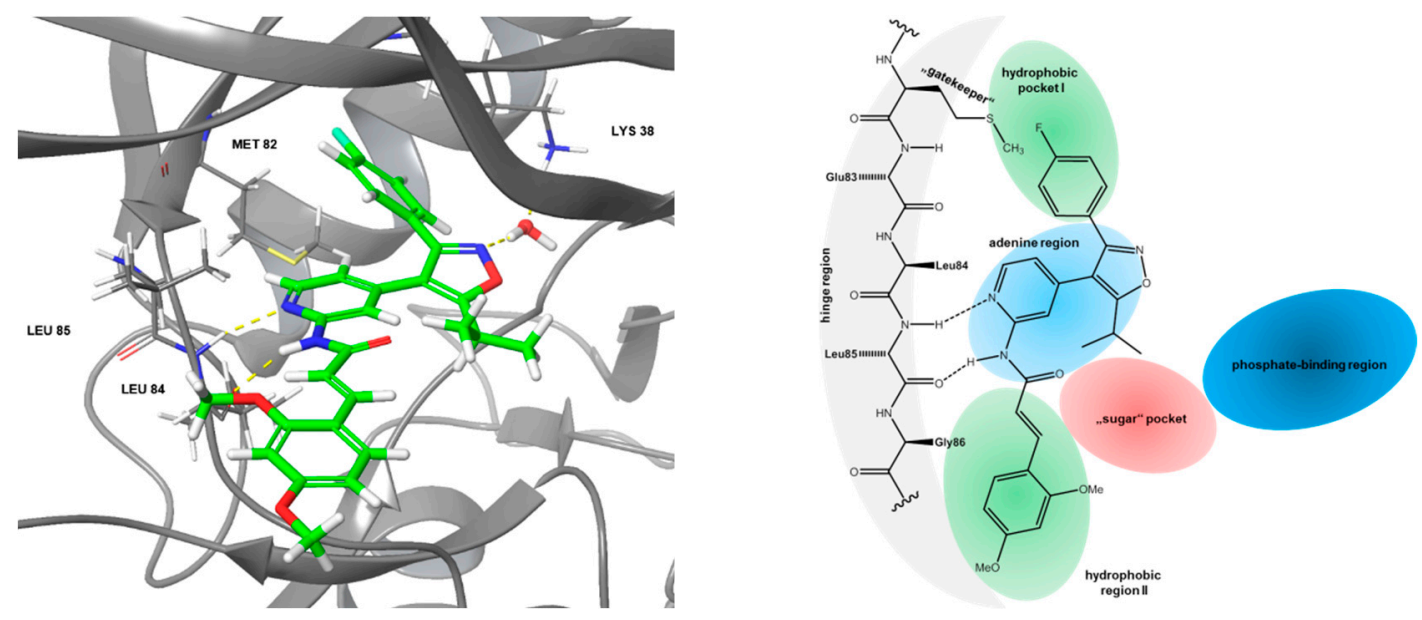

Figure 3. Left: Predicted binding mode of lead compound 8 within the ATP binding pocket of protein kinase CK1 (grey cartoon, pdb 3UZP [41]); hydrogen bond interactions, yellow dashed lines. Right: Schematic ligand-interaction-diagram of isoxazole 8 according to Traxler model.

To extend the pharmacophore of lead compound 8 towards the ribose pocket we replaced the susceptible double bond with a five-membered pyrrole ring to mimic and to rigidify the E-configured cinnamic acid moiety. Furthermore, the pyrrole nitrogen was used as a handle to attach the chiral scaffolds (Scheme 1). Docking of the designed ligands into CK1 $\delta$ revealed that the key pharmacophore interactions described above are maintained. Additionally, the chiral scaffolds are predicted to pack between Ile-15 and Asp-91 and to crosslink the $\beta 1 b$ strand of the $\mathrm{N}$-terminal lobe and the $\alpha \mathrm{B}$ helix of the

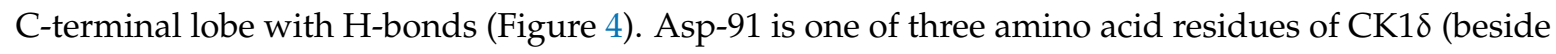


Asp-132 and Ser-88) that are deemed to be involved in ribose binding of ATP [42,43]. While compound $29 \mathrm{~d}$ is predicted to form $\mathrm{H}$-bonds between its hydroxyl groups and the carboxyl group of Asp-91 (Figure 4A), enantiomer 29e is predicted to bind to Asp-91 with the NH-group of the pyrrolidine ring (Figure 4B). Moreover, H-bonding to the N-terminal carbonyl group of Ile-15 is predicted for both enantiomers. However, a clear stereochemical preference was not observed by scoring function [GScore -14.1 (29d) vs -13.8 (29e)].

A

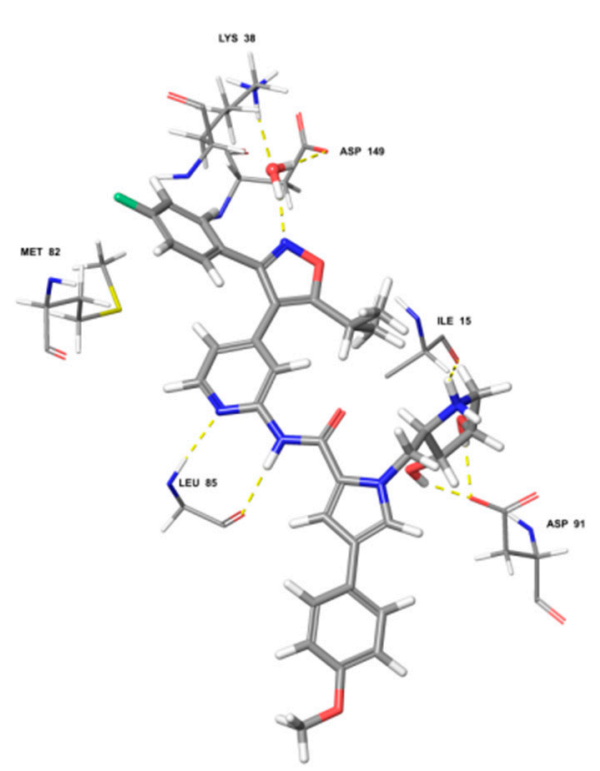

B

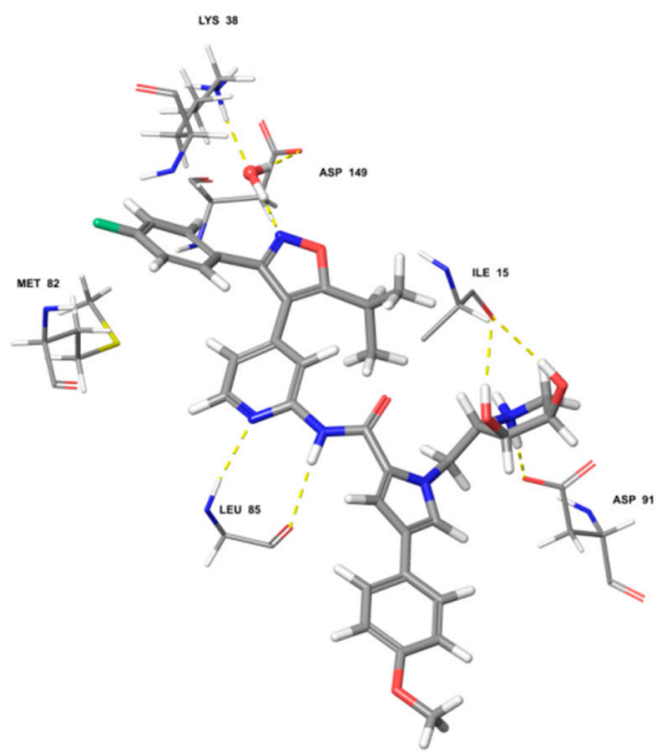

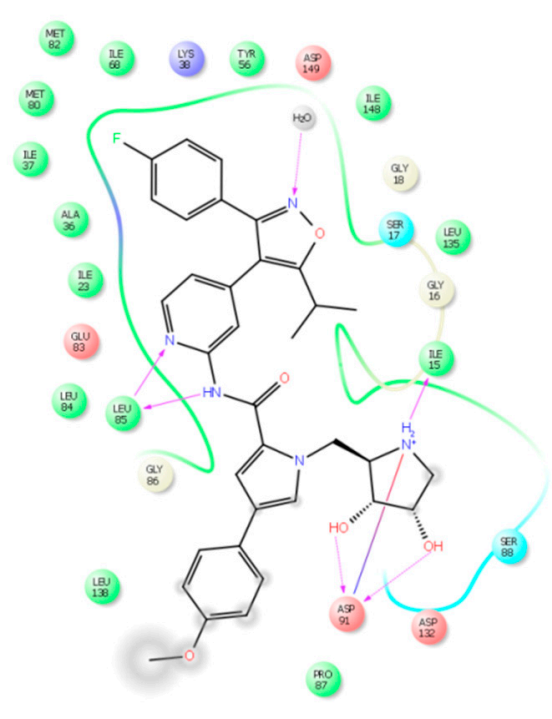

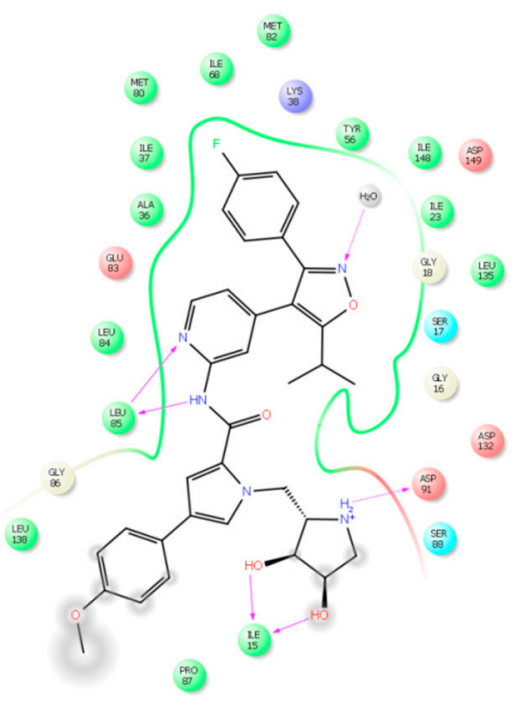

Figure 4. Predicted binding modes (left) and their corresponding ligand-interaction-diagrams (right) of designed enantiomers 29d (A) and 29e (B) (grey sticks) within the ATP binding site of protein kinase CK1ס (grey lines, pdb 3UZP [41]); potential hydrogen bond interactions, yellow dashed-lines.

\subsection{Chemistry}

The synthesis of our envisaged inhibitors began with the preparation of the two enantiomerically pure iminoribitol derivatives (-)-20 and (+)-20 (Scheme 2). Whilst (+)-20 was solely prepared from D-methionine its enantiomer (-)-20 was either synthesized from L-methionine or, particularly at larger scale, from the available 2,3-O-isopropylidene-L-lyxono-1,4-lactone, following published procedures [44-49]. Starting from the amino acids, D- and L-methionine were firstly converted 
into the corresponding tert-Butyloxycarbonyl (Boc) protected derivatives [50]. Upon reduction of the carboxylic acid functions [51] and subsequent tert-butyldiphenylsilyl (TBDPS)-protection of the primary alcohols $(S)-\mathbf{1 0}$ and $(R)-\mathbf{1 0}$ [52] sulfides $(S)-\mathbf{1 1}$ and $(R)-\mathbf{1 1}$ were oxidized to the sulfoxides (S)-12 and (R)-12 with meta-chloroperoxybenzoic acid ( $m$-CPBA) [53,54]. Thermal syn-elimination [52] of sulfoxides $(S)-\mathbf{1 2}$ and (R)-12 yielded the vinylglycinol intermediates $(S)-\mathbf{1 4}$ and $(R)-\mathbf{1 4}$ which underwent $N$-allylation to $(S)-\mathbf{1 5}$ and $(R)-\mathbf{1 5}$ when reacted with sodium hydride and allyl bromide. The allylation reaction, however, turned out to be capricious as higher yields were only achieved by repeated addition of the same amounts of sodium hydride and allyl bromide. Attempts to deploy potassium tert-butoxide as a base did not lead to any satisfactory product formation in our hands and other reaction conditions were reported to be unsuccessful $[55,56]$. The intramolecular cross-metathesis reaction of the $N$-allylvinylglycinol precursors (S)-15 and (R)-15 using benzylidene-bis(tricyclohexylphosphino)dichlororuthenium (Grubbs $1^{\text {st }}$-generation catalyst) then yielded the 3,4-dehydroprolinol intermediates $(S)-\mathbf{1 6}$ and (R)-16 [55-57]. The optical purity of the vinylglycinol intermediates $(S)-\mathbf{1 4}$ and $(R)-\mathbf{1 4}$ and the 3,4-dehydroprolinol intermediates $(S)-\mathbf{1 6}$ and (R)-16 was analyzed by asymmetric high-performance liquid chromatography (HPLC) and determined to be $>99 \%$. Employing established protocols $(S)-\mathbf{1 6}$ and $(R)-\mathbf{1 6}$ were then dihydroxylated with catalytic osmium tetroxide in the presence of 4-methylmorpholine $\mathrm{N}$-oxide (NMO) and the so formed diols (-)-17 and (+)-17 were subsequently acetal protected with 2,2-dimethoxypropane (DMP) in the presence of catalytic amounts of para-toluenesulfonic acid ( $\mathrm{p}-\mathrm{TSOH})$ to afford the fully protected iminoribitol derivatives (-)-18 and (+)-18 [57,58]. Finally, the silyl protecting groups were cleaved with tetra- $n$-butylammonium fluoride (TBAF) and the resulting primary alcohols $(-)-19$ and (+)-19 were activated as their mesylates $(-)-20$ and $(+)-20$ for coupling with the pyrrole building blocks $\mathbf{2 4 a - c}$ (Scheme 3) [47-49].
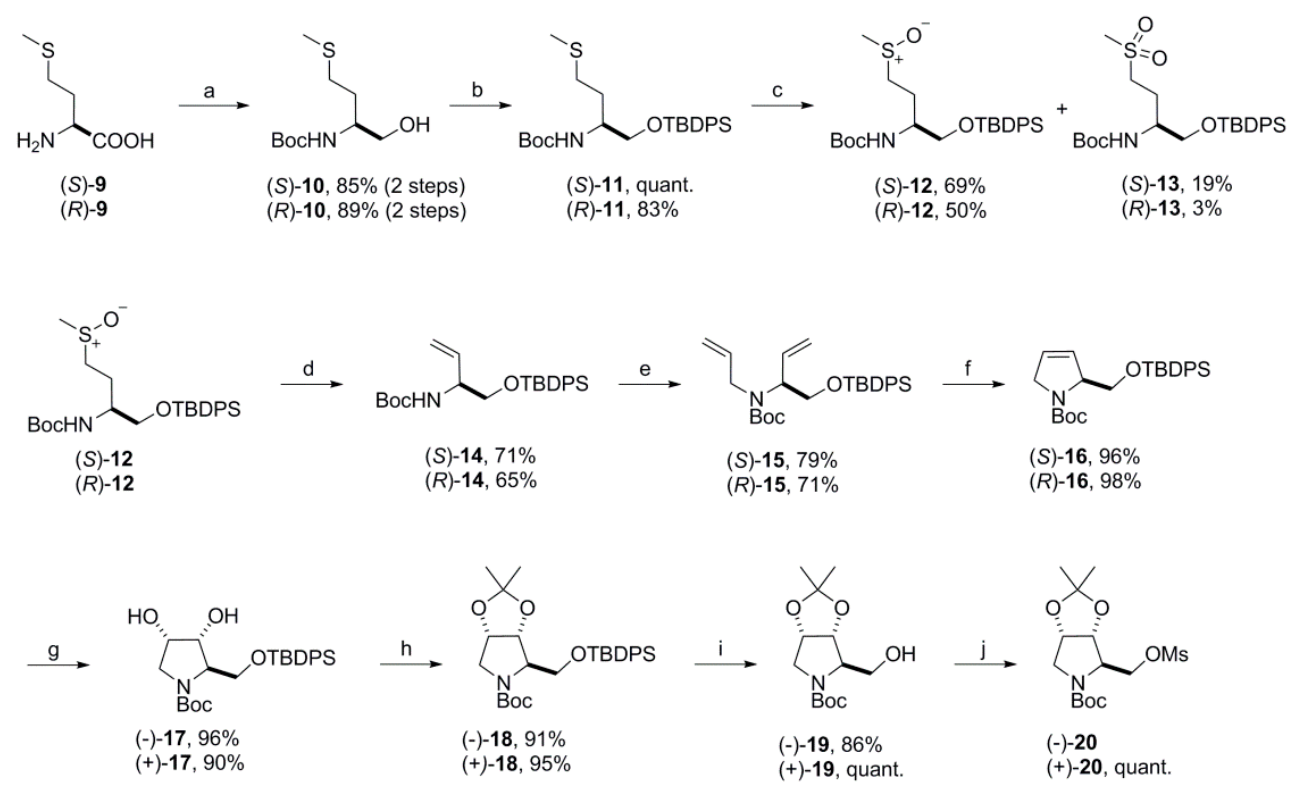

Scheme 2. (a) 1. $1 \mathrm{M} \mathrm{NaOH}_{\mathrm{aq}}$, Di-tert-butylcarbonate $\left(\mathrm{Boc}_{2} \mathrm{O}\right), 1,4$-dioxane, $0{ }^{\circ} \mathrm{C}, 3.5 \mathrm{~h}$ [50]; 2. $N$-methylmorpholine, ClCOOEt, THF, $-20^{\circ} \mathrm{C}$; $\mathrm{NaBH}_{4}, \mathrm{MeOH}, 0{ }^{\circ} \mathrm{C}$ [51]; (b) TBDPSCl, imidazole., DMF, rt; (c) m-CPBA, DCM; (d) o-dichlorobenzene, $\mathrm{CaCO}_{3}, \Delta, 7 \mathrm{~h}$; (e) NaH, allyl bromide, DMF, rt; (f) Grubbs' Ist gen. cat., DCM, rt; (g) $\mathrm{OsO}_{4}, \mathrm{NMO}$, acetone/water, rt; (h) DMP, acetone, $p-\mathrm{TsOH}^{\text {st }} \mathrm{H}_{2} \mathrm{O}$; (i) TBAF, THF, rt; (j) methanesulfonyl chloride (MsCl), $\mathrm{NEt}_{3}, \mathrm{DCM}$. 


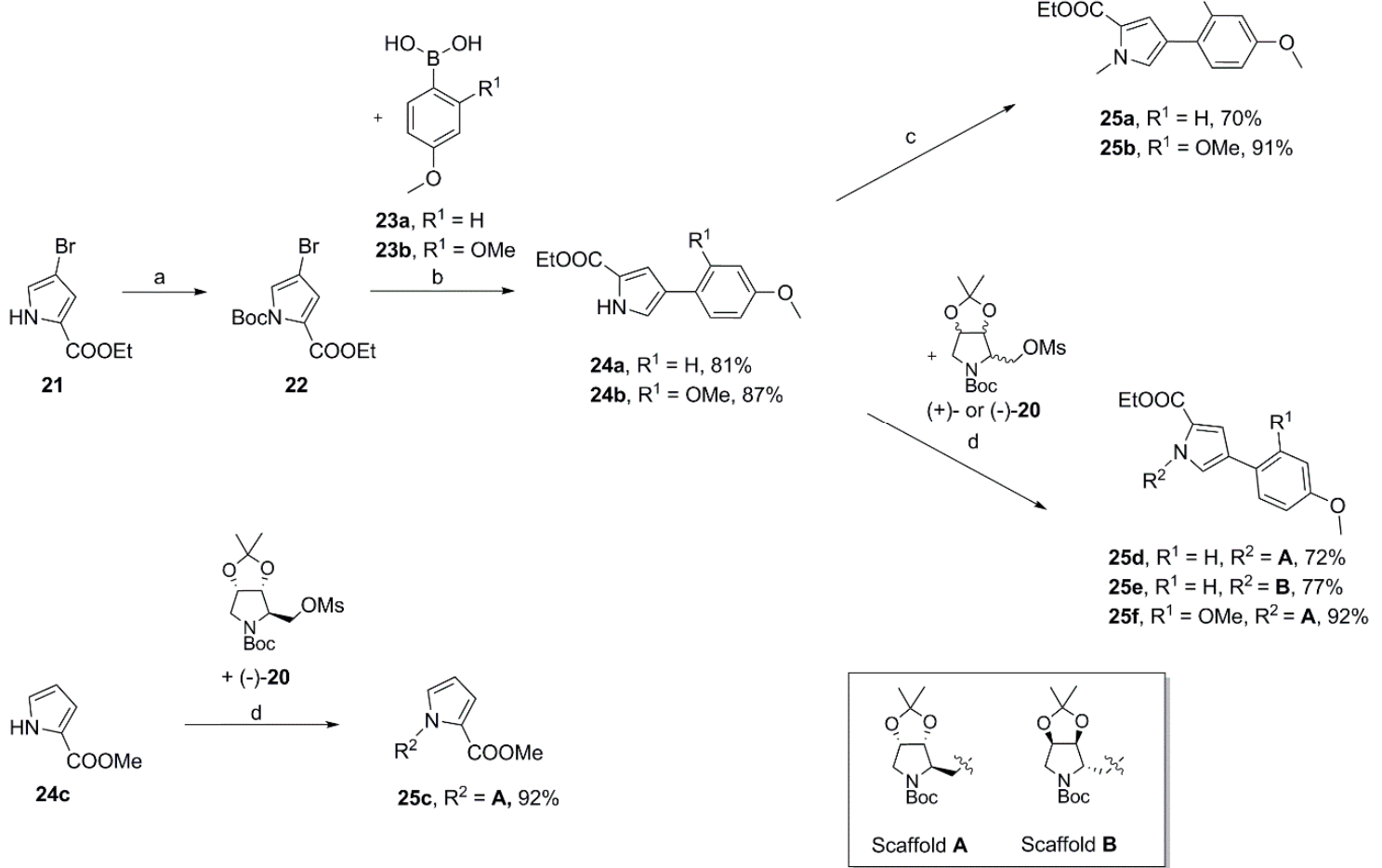

Scheme 3. (a) $\mathrm{Boc}_{2} \mathrm{O}, \mathrm{N}, \mathrm{N}$-dimethylpyridin-4-amine (DMAP), $\mathrm{CH}_{3} \mathrm{CN}, 1 \mathrm{~h}$ [59]; (b) $5 \mathrm{~mol} \%$ $\mathrm{Pd}\left(\mathrm{PPh}_{3}\right)_{4}, \mathrm{DMF}, 2 \mathrm{M} \mathrm{Na} 2 \mathrm{CO}_{3 \mathrm{aq}}, 110{ }^{\circ} \mathrm{C}, 24 \mathrm{~h}$; (c) MeI, NaH, DMF, $2.5 \mathrm{~h}, 0{ }^{\circ} \mathrm{C}$-rt; (d) $\mathrm{Cs}_{2} \mathrm{CO}_{3}$, tetra- $n$-butylammonium iodide (TBAI), DMF, $80{ }^{\circ} \mathrm{C}$.

The required pyrrole building blocks $\mathbf{2 4 a} \mathbf{a} \mathbf{b}$ (Scheme 3) were prepared from commercially available ethyl 4-bromopyrrole-2-carboxylate (21) in two steps following procedures published by Handy et al. [40,59-61]. Previous studies showed that the use of unprotected pyrrole 21 in the envisaged Suzuki-Miyaura coupling results in extensive dehalogenation [61]. Accordingly, pyrrole 21 was initially $\mathrm{N}$-Boc protected and subjected to palladium-catalyzed coupling with boronic acids 23a and $\mathbf{2 3 b}$ to give the desired pyrrole building blocks $24 \mathrm{a}$ and $\mathbf{2 4 b}$. Now, mesylates (-)-20 and (+)-20, and pyrrole building blocks $24 \mathbf{a}, \mathbf{b}$ were joint together in the presence of cesium carbonate at $80{ }^{\circ} \mathrm{C}$ to yield compounds $\mathbf{2 5 d} \mathbf{d} \mathbf{f}$ (Scheme 3) [62]. The enantiomeric purity of intermediates $\mathbf{2 5 d}$ and $\mathbf{2 5 e}$ was analyzed by asymmetric HPLC and determined to be $>99 \%$. In addition, the three-dimensional structure of $25 \mathrm{~d}$ was confirmed by X-ray crystallographic analysis (Figure S1). To synthesize inhibitor 29c without aryl substituent attached to the pyrrole ring $1 \mathrm{H}$-pyrrole-2-carboxylate 24c [63] was coupled with mesylate (-)-20 employing the same methodology as before to afford compound 25c. Furthermore, we aimed to prepare inhibitors $\mathbf{2 8 a} \mathbf{a} \mathbf{b}$ in which the iminosugar unit is replaced by a methyl group. For this purpose, pyrrole building blocks $\mathbf{2 4} \mathbf{a}, \mathbf{b}$ were methylated under standard reaction conditions to give compounds $25 \mathbf{a}, \mathbf{b}$.

Following saponification of the esters $25 a-f$ the isoxazole building block 30 [34] was coupled stepwise to the corresponding carboxylic acids $26 \mathbf{a}-\mathbf{f}$ via their $N$-hydroxybenzotriazole (HOBt)-activated esters $\mathbf{2 7 a}-\mathbf{f}$ (Scheme 4) [64]. Thus, coupling products $\mathbf{2 8 a}-\mathbf{f}$ were obtained in yields of up to $67 \%$. Finally, cleavage of the protecting groups under acidic reaction conditions furnished $\mathbf{2 9 c}-\mathbf{f}$. 


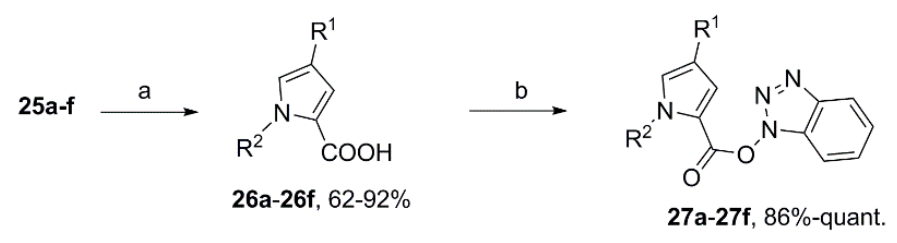

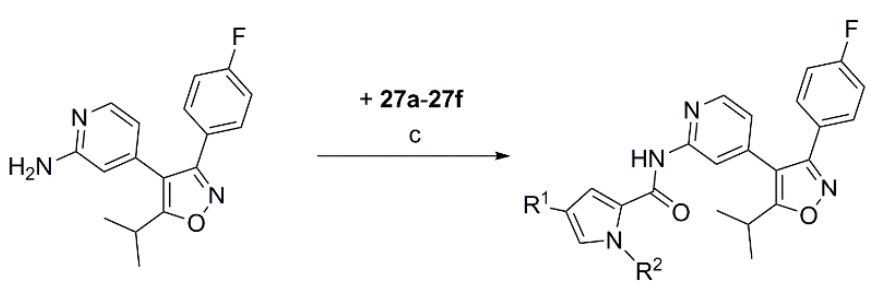

30 28a-28f, 36-67\%

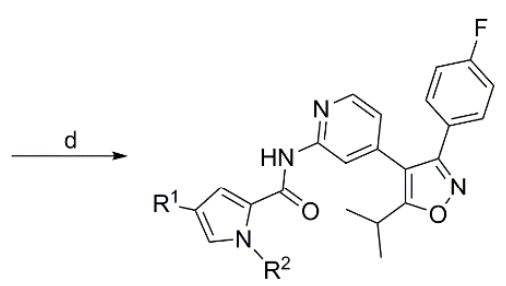

29c, $R^{1}=H, R^{2}=C, 84 \%$

29d, $R^{1}=4-\mathrm{MeO}-\mathrm{Ph}, \mathrm{R}^{2}=\mathrm{C}, 77 \%$

29e, $R^{1}=4-\mathrm{MeO}-\mathrm{Ph}, \mathrm{R}^{2}=\mathrm{D}, 79 \%$

29f, $R^{1}=2,4-(\mathrm{MeO})_{2}-\mathrm{Ph}, \mathrm{R}^{2}=\mathrm{C}, 64 \%$
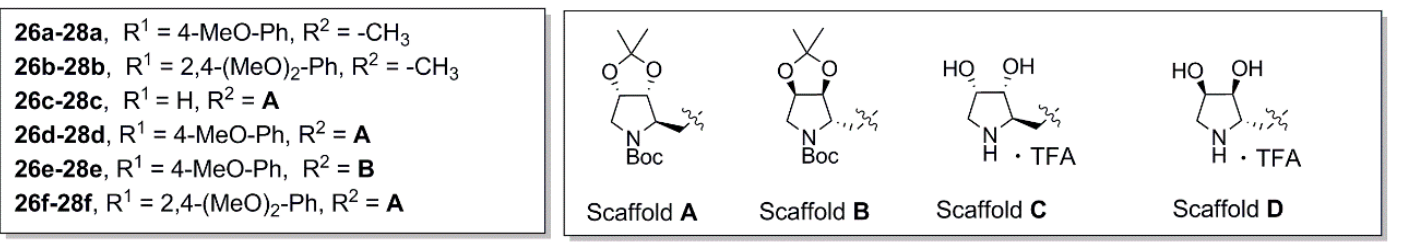

Scheme 4. (a) $4 \mathrm{M} \mathrm{NaOH}_{\mathrm{aq}}$ or $8 \mathrm{M} \quad \mathrm{KOH}_{\mathrm{aq}}$, THF/MeOH (2:1) $50-60 \quad{ }^{\circ} \mathrm{C} ; \quad$ (b) $\mathrm{N}, \mathrm{N}, \mathrm{N}^{\prime}, \mathrm{N}^{\prime}$-tetramethyl-O-(1H-benzotriazol-1-yl)uronium hexafluorophosphate (HBTU), DMF, $\mathrm{NEt}_{3}, \mathrm{rt}$; (c) NaH, DMF, rt; (d) trifluoroacetic acid (TFA)/water $(9: 1), 0{ }^{\circ} \mathrm{C}, 3 \mathrm{~h}$.

\subsection{Biological Evaluation}

Compounds $\mathbf{2 8 a}-\mathbf{c}$ and $\mathbf{2 9 d}-\mathbf{f}$ were initially screened in in-vitro kinase assays at a concentration of $10 \mu \mathrm{M}$ for their ability to inhibit CK1 $\delta$ and CK1 1 . Compounds that showed only low residual activity at this concentration were taken forward to measure $\mathrm{IC}_{50}$ values (Table 1 ). In accordance with our molecular modelling studies 29d and 29e showed high activity in the CK1 $\delta$ and CK1 $\varepsilon$ in-vitro kinase assays with $\mathrm{IC}_{50}$ values in the nanomolar range. Interestingly, no preference was observed between chiral moieties of $\mathbf{2 9 d}$ and $\mathbf{2 9 e}$, while replacement of the pyrrolidine scaffolds by a methyl (28a and 28b) ablated activity against CK1 $\delta$ and CK1 $\varepsilon$. Omitting the aryl moiety (29c) also resulted in a considerable decrease in activity relative to $29 \mathrm{~d}$ and $29 \mathrm{e}$ in accordance with previously reported CK1 $\delta$ inhibitors [40]. Taken together, both the aryl moiety and the pyrrolidine scaffold seem to contribute to the binding affinity. On the one hand, interactions between the pyrrolidine scaffold and the active site might could play an important role in determining inhibitor engagement with the active site. On the other hand, the molecular configuration of the scaffolds (29d vs 29e) does not seem to contribute to affinity and CK1-isoform selectivity. 
Table 1. Activity and $\mathrm{IC}_{50}$ values of compounds $28 \mathbf{a}, \mathbf{b}$ and $29 \mathrm{c}-\mathbf{f}$ in $\mathrm{CK} 1 \delta$ and $\mathrm{CK} 1 \varepsilon$ kinase assays. Results are presented as mean \pm SD from experiments performed in triplicate $(n=3)$.

\begin{tabular}{|c|c|c|c|c|c|c|}
\hline$\#$ & $\mathbf{R}^{1}$ & $\mathbf{R}^{2}$ & \multicolumn{2}{|c|}{ CK1ठ } & \multicolumn{2}{|c|}{ CK1ع } \\
\hline & & & $\begin{array}{c}\text { Residual } \\
\text { Activity [\%] } \\
\text { at } 10 \mu \mathrm{M} \pm \mathrm{SD}\end{array}$ & $\begin{array}{l}\mathrm{IC}_{50}[\mu \mathrm{M}] \\
\quad \pm \mathrm{SD}\end{array}$ & $\begin{array}{c}\text { Residual } \\
\text { Activity [\%] } \\
\text { at } 10 \mu \mathrm{M} \pm \mathrm{SD}\end{array}$ & $\begin{array}{l}\mathrm{IC}_{50}[\mu \mathrm{M}] \\
\quad \pm \mathrm{SD}\end{array}$ \\
\hline $28 a$ & & $\mathrm{Me}$ & $48 \pm 7$ & n.d. & $70 \pm 21$ & n.d. \\
\hline $28 b$ & & $\mathrm{Me}$ & $48 \pm 4$ & n.d. & $47 \pm 11$ & n.d. \\
\hline $29 c$ & $\mathrm{H}$ & & $7.7 \pm 0.2$ & $0.45 \pm 0.07$ & $18 \pm 1$ & $1.5 \pm 0.3$ \\
\hline $29 d$ & & & $2.9 \pm 0.3$ & $0.038 \pm 0.003$ & $12 \pm 2$ & $0.065 \pm 0.012$ \\
\hline $29 \mathrm{e}$ & & & $3.4 \pm 0.7$ & $0.047 \pm 0.004$ & $14 \pm 3$ & $0.063 \pm 0.009$ \\
\hline $29 f$ & & & $2.8 \pm 0.5$ & $0.037 \pm 0.004$ & $9.7 \pm 1.6$ & $0.1 \pm 0.014$ \\
\hline
\end{tabular}

In addition to the in-vitro kinase assays, all compounds were tested in cell viability assays against tumor cell lines. Since inhibition of CK1 $1 \delta$ prolongs the survival of SV40 T-Ag/mutant CK1 $\delta$ bitransgenic mice [65] and overexpression of CK1 correlates with reduced survival rates of colorectal and breast cancer patients [66], we chose HT-29 and MCF-7 cells for biological testing [67,68]. These two cell lines are characterized on a molecular level in detail, exhibiting alterations in WNT and p53 signaling pathways, both being regulated by CK1 isoforms [30,69]. All compounds were initially screened at $5 \mu \mathrm{M}, 10 \mu \mathrm{M}$ and $20 \mu \mathrm{M}$, respectively (Table 2). $\mathrm{EC}_{50}$ values were determined for compounds that showed a clear reduction in cell viability. There is a trend between the $\mathrm{EC}_{50}$ data for both cell lines and enzyme inhibitory data against CK1, with compounds $29 \mathbf{d}-\mathbf{f}$ exhibiting a modest effect on both, HT-29 and MCF-7 cell viability. These results are consistent with previous findings that inhibitory effects of CK1 specific inhibitors are dependent on the cellular background [29,70-72]. Especially alterations in WNT and p53 signaling influence the effects of CK1 specific inhibitors [30,73]. The modest inhibitory effect on cancer cell viability may have several reasons: (i) The hydrophilic character of the pyrrolidine scaffolds might reduce their cellular uptake; (ii) efflux systems could contribute to a decreased availability of the inhibitors, (iii) compounds could be partly metabolized (e.g., the hydroxy groups of the pyrrolidine scaffolds) and, (iv) since these inhibitors are ATP competitive inhibitors and cellular ATP concentrations are much higher than the ATP concentration used in the in-vitro assays, higher compound concentrations are necessary to inhibit CK1 $\delta$ within cells. 
Table 2. EC $_{50}$ values and cell viability of MCF-7 and HT-29 cells after treatment with compounds $\mathbf{2 8 a}, \mathbf{b}$ and 29c-f. Results are presented as mean \pm SD from experiments performed in triplicate $(n=3)$.

\begin{tabular}{|c|c|c|c|c|c|c|c|c|}
\hline \multirow{3}{*}{$\#$} & \multicolumn{4}{|c|}{ MCF-7 } & \multicolumn{4}{|c|}{ HT-29 } \\
\hline & \multicolumn{3}{|c|}{ Cell Viability [\%] } & \multirow{2}{*}{$\begin{array}{l}\mathrm{EC}_{50} \\
{[\mu \mathrm{M}]}\end{array}$} & \multicolumn{3}{|c|}{ Cell Viability [\%] } & \multirow{2}{*}{$\begin{array}{l}\mathrm{EC}_{50} \\
{[\mu \mathrm{M}]}\end{array}$} \\
\hline & $5 \mu \mathbf{M}$ & $10 \mu \mathrm{M}$ & $20 \mu \mathrm{M}$ & & $5 \mu \mathbf{M}$ & $10 \mu \mathrm{M}$ & $20 \mu \mathrm{M}$ & \\
\hline $28 a$ & $103 \pm 16$ & $88 \pm 9$ & $54 \pm 5$ & n.d. & $111 \pm 19$ & $98 \pm 22$ & $78 \pm 10$ & n.d. \\
\hline $28 b$ & $89 \pm 13$ & $84 \pm 11$ & $79 \pm 12$ & n.d. & $94 \pm 23$ & $59 \pm 14$ & $52 \pm 10$ & n.d. \\
\hline $29 \mathrm{c}$ & $90 \pm 10$ & $81 \pm 5$ & n.d. & $39 \pm 12$ & $80 \pm 16$ & $78 \pm 11$ & n.d. & $24 \pm 6$ \\
\hline $29 d$ & $100 \pm 16$ & $76 \pm 19$ & $5.6 \pm 1.5$ & $10 \pm 2$ & $76 \pm 13$ & $51 \pm 8$ & $14 \pm 2$ & $8.0 \pm 1.7$ \\
\hline $29 e$ & $90 \pm 27$ & $58 \pm 18$ & $8.0 \pm 3.4$ & $10 \pm 2$ & $75 \pm 13$ & $42 \pm 10$ & $11 \pm 3$ & $8.8 \pm 0.8$ \\
\hline $29 f$ & $87 \pm 5$ & $33 \pm 7$ & $3.9 \pm 0.2$ & $9.6 \pm 0.8$ & $50 \pm 10$ & $25 \pm 5$ & $9.4 \pm 3.0$ & $5.4 \pm 0.4$ \\
\hline
\end{tabular}

Next, we screened effective inhibitor 29d in a panel of 320 kinases at a concentration of $1 \mu \mathrm{M}$

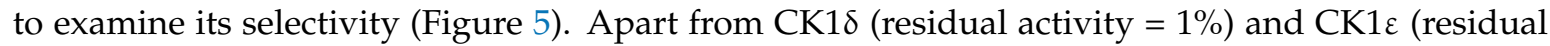
activity $=4 \%) 29 \mathrm{~d}$ hits only four other kinases, namely CK1 $\alpha$ (residual activity $=16 \%)$, JNK2 $(40 \%)$, JNK3 $(15 \%)$, p38 $\alpha(17 \%)$ thus resulting in an excellent selectivity score of 0.02 (number of kinases with residual activity $<50 \%$ / total number of tested kinases).
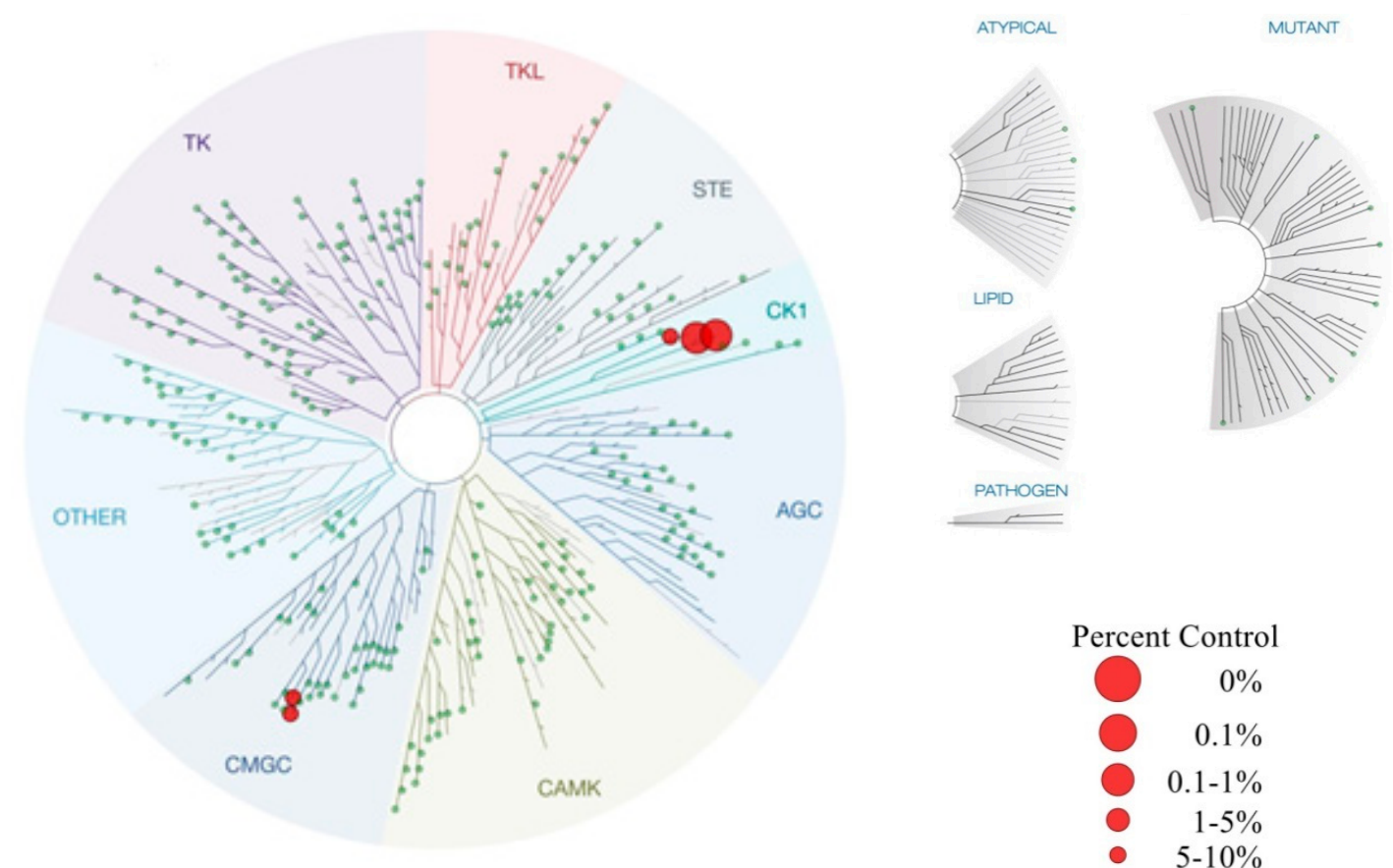

Figure 5. Dendrogram representation of the selectivity profile of $29 \mathrm{~d}$ at a concentration of $1 \mu \mathrm{M}$ in a panel of 320 wild-type kinases (ProQinase, Freiburg, Germany). The residual kinase activity was determined compared to DMSO control. Images were generated using TREEspot Software Tool, DISCOVERX CORPORATION 2010. The complete raw data are shown in Table S2.

\subsection{X-ray Analysis of ligand-CK1 $1 \delta$ Complexes}

In the next step we sought to elucidate structural details of the binding of our chiral inhibitors via

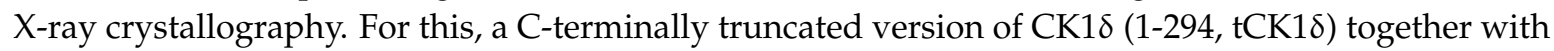
$29 \mathrm{~d}$ and 29e, respectively, were used in co-crystallization trials. Crystals suitable for diffraction analysis grew in presence of both compounds in crystallization conditions containing $0.1 \mathrm{M} \mathrm{MES} \mathrm{(pH=5.5),}$ $10 \%$ polyethylengylcol (PEG) 4000 and $0.2 \mathrm{M} \mathrm{Li}_{2} \mathrm{SO}_{4}$. These crystals diffracted to a resolution limit of 
$1.8 \AA$ and belong to the monoclinic crystal system. In each asymmetric unit, two tCK1 molecules were present with an isoxazole-based ligand bound to each ATP binding pocket (Figure 6).

A

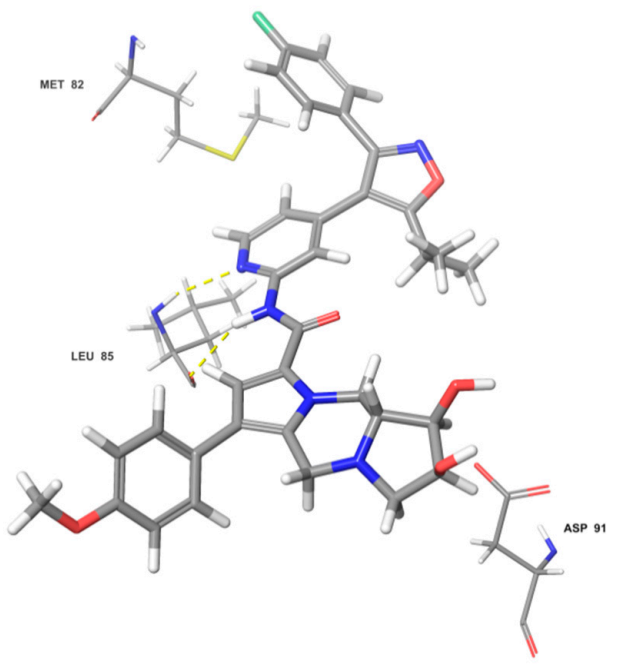

B

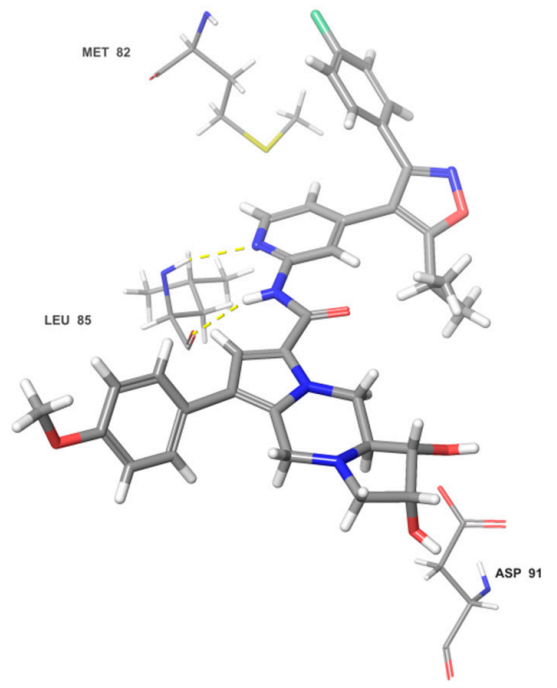

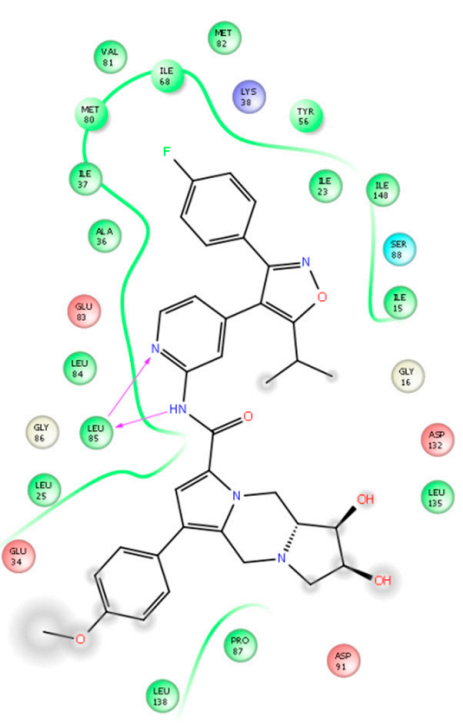

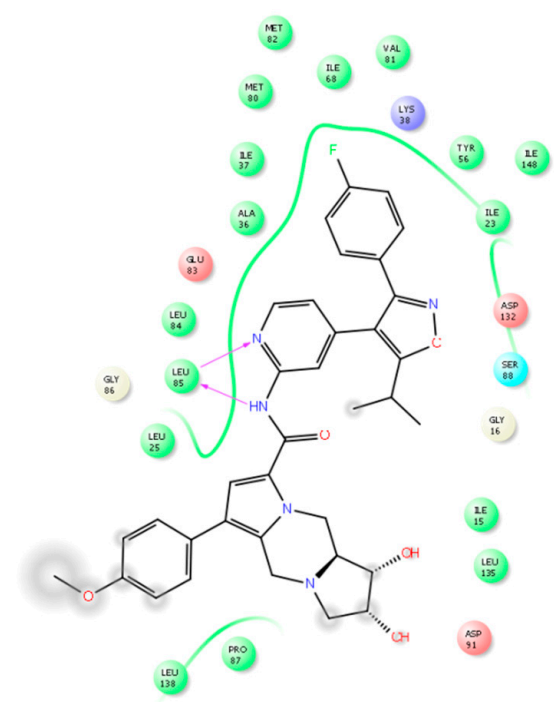

Figure 6. Binding modes of compounds $31 \mathrm{a}(\mathrm{A})$ and $\mathbf{3 1 b}(\mathrm{B})$ as determined by X-ray crystallographic analysis of respective ligand-protein complexes. Representation of their binding pose (left) and ligand-interaction diagram (right). Key interactions are shown.

Surprisingly, the ligands in both protein-ligand complexes proved to be different from the actual target compounds submitted to co-crystallization. Since all analytical data for the originally employed inhibitors was consistent with the given structures, we postulate that the incorporation of an additional methylene group occurred via spontaneous Pictet-Spengler cyclization [74-76] during crystallization. In line with this notion, PEG, which has been used in our soaking solutions, is known to generate formaldehyde traces that have been shown in the past to affect the derivatization of both, proteins and ligands [77-79]. To further corroborate this hypothesis, we examined whether simple exposure of $29 \mathrm{~d} / \mathbf{e}$ to formaldehyde would give rise to the same Pictet-Spengler products $31 \mathbf{a} / \mathbf{b}$ (Scheme 5). Indeed, when compounds $29 \mathrm{~d} / \mathbf{e}$ were treated with formaldehyde at room temperature $31 \mathrm{a} / \mathrm{b}$ were formed readily and the obtained spectroscopic data was consistent with the predicted Pictet-Spengler products. In addition, aqueous solutions of $29 \mathrm{~d}$ and $29 \mathrm{e}$ with and without PEG were analyzed by liquid chromatography-mass spectrometry (LC-MS) over a period of 7 days. Only in those solutions 
that had PEG present significant product formation of $\mathbf{3 1 a}$ and $\mathbf{3 1} \mathbf{b}$ was observed indicating that the degradation of PEG may have been the likely source of formaldehyde.

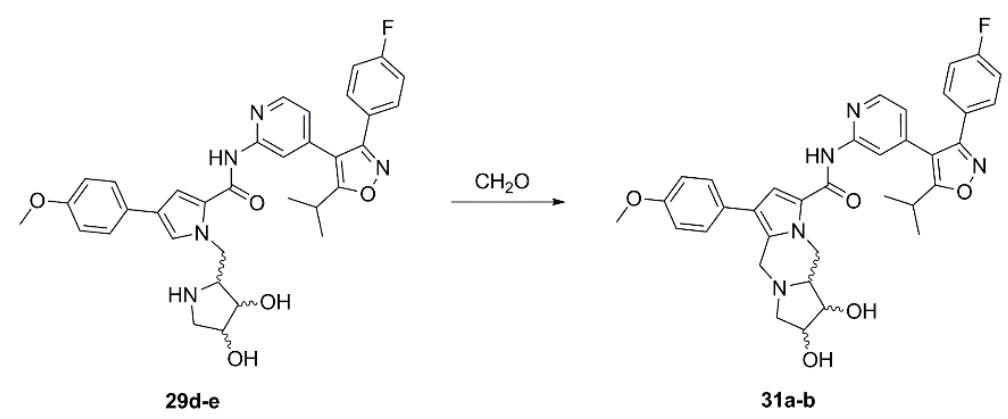

Scheme 5. Proposed Pictet-Spengler reaction of originally employed compounds $29 \mathrm{~d}$ and $29 \mathrm{e}$ with formaldehyde impurities to yield new compounds 31a and 31b. Traces of formaldehyde may originate from PEG reagent employed in ligand soaking approaches.

Following the discovery of new ligands as artefacts in the X-ray complexes, both these Pictet-Spengler products $31 \mathrm{a}$ and $\mathbf{3 1} \mathbf{b}$ were re-synthesized and subsequently assessed for their biological activities (Table 3). In the in-vitro kinase assays both compounds exhibited nanomolar activity against $\mathrm{CK} 1 \delta$ and $\mathrm{CK} 1 \varepsilon$ with a five-fold preference for $\mathrm{CK} 1 \delta$ over $\mathrm{CK} 1 \varepsilon$. The cellular assays revealed $31 \mathrm{a}$ and $31 \mathrm{~b}$ to be more potent than the originally designed compounds $29 \mathrm{~d}$ and $29 \mathrm{e}$ with $\mathrm{EC}_{50}$ values below $2 \mu \mathrm{M}$. The higher cellular potency of both compounds is in a way surprising, because in contrast to compounds $29 \mathrm{~d}$ and $29 \mathrm{e}$ the chiral moieties of the Pictet-Spengler products 31a and $\mathbf{3 1 b}$ are too far away to interact with residues in the ATP binding site (Figure 6). Further studies will need to elucidate compound's kinase selectivity, and also their specificity in terms of addressing other targets. However, our results suggest that the chiral pyrrolidine scaffold attached to kinase inhibitors (and beyond) can be suitable for medicinal chemistry applications.

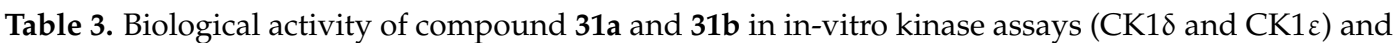
cellular assays (MCF-7- and HT-29). Results are presented as mean \pm SD from experiments performed in triplicate $(n=3)$.

[




\section{Materials and Methods}

\subsection{Molecular Modelling}

Molecular modelling was performed on a DELL Precision T3610 four core workstation using Schrödinger Maestro, version 10.4, 2015, Schrödinger, LLC, New York, NY, USA. The protein crystal structure of CK1 $\delta$ was used from the RCSB protein data bank www.rcsb.org (pdb 3UZP [41]) and prepared with the Protein Preparation Wizard 2015-4 (Epik version 2.4, Schrödinger, LLC, 2015; Impact version 5.9, Schrödinger, LLC, 2015; Prime version 3.2, Schrödinger LLC, 2015) regarding assignment of bond orders, addition of hydrogen atoms, identification of disulfide bonds and conversion of artificial selenomethionines to methionines (default settings) [80]. Designed ligands were minimized with MacroModel, version 11.0, Schrödinger, LLC, 2015 using an OPLS2005 force field. Ligand docking and receptor grid generation were performed with Glide, version 6.9, Schrödinger, LLC, 2015 using the standard protocol [81-83].

\subsection{Chemistry}

\subsubsection{General Experimental Procedures}

Melting points were determined either on a Stuart Melting Point (SMP3) apparatus and are uncorrected, or by differential scanning calorimetry (DSC) on a Mettler Toledo DSC1 instrument at a heating rate of $10 \mathrm{~K} \cdot \mathrm{min}^{-1}$. Proton $\left({ }^{1} \mathrm{H}\right)$ and carbon $\left({ }^{13} \mathrm{C}\right) \mathrm{NMR}$-spectra were recorded on Bruker Avance (III)-500 or Avance (I)-300 spectrometers. ${ }^{19} \mathrm{~F}$ NMR spectra were recorded at $470 \mathrm{MHz}$ and are reported unreferenced. Chemical shifts are reported in ppm relative to $\mathrm{Me}_{4} \mathrm{Si}$ (TMS, $\delta 0$ ), or residual solvent peaks as an internal standard set to $\delta 7.26$ and $77.00\left(\mathrm{CDCl}_{3}\right)$, or $\delta 3.34$ and 49.05 (MeOD), or $\delta 2.50$ and $39.43\left(d_{6}\right.$-DMSO). NMR data is reported as follows: Chemical shift in ppm, multiplicity (ap = apparent, $\mathrm{s}=$ singlet, $\mathrm{d}=$ doublet, $\mathrm{t}=$ triplet, $\mathrm{q}=$ quartet, $\mathrm{sp}=$ septet, $\mathrm{br}=$ broad, $\mathrm{dd}=\mathrm{doublet}$ of doublets, $\mathrm{td}=$ triplet of doublets, $\mathrm{dt}=$ doublet of triplets, $\mathrm{m}=$ multiplet), coupling constant in $\mathrm{Hz}$, integration.

Electrospray ionization (ESI) mass spectrometry (MS) experiments were performed on a QTOF Premier mass spectrometer (Micromass, UK) under normal conditions. Sodium formate solution was used as a calibrant for high resolution mass spectra (HRMS) measurements. Elemental microanalyses were performed at The Campbell Microanalytical Laboratory, Department of Chemistry at the University of Otago on a Carlo-Erba EA 1108 elemental analyzer. Specific optical rotations were acquired on a Rudolph Autopol ${ }^{\circledR}$ IV Automatic polarimeter at ambient temperature $\left(20{ }^{\circ} \mathrm{C}\right)$, unless otherwise stated, $\lambda=589 \mathrm{~nm}$ and concentration $(\mathrm{g} / 100 \mathrm{~mL})$ in the solvent indicated, using a cell of $100 \mathrm{~mm}$ path length.

All reactions were monitored by thin layer chromatography (TLC) using $0.2 \mu \mathrm{m}$ silica gel (Merck Kieselgel $60 \mathrm{~F}_{254}$ ) precoated aluminium plates, using UV light, ammonium molybdate, ninhydrin or potassium permanganate staining solution to visualize. Flash column chromatography was performed on Davisil ${ }^{\circledR}$ silica gel (60, particle size $0.040-0.063 \mathrm{~mm}$ ), or using Reveleris ${ }^{\circledR}$ silica or C-18 reversed phase flash cartridges on a Grace Reveleris ${ }^{\circledR}$ automated flash system with continuous gradient facility. Solvents for reactions and chromatography were analytical grade and were used as supplied unless otherwise stated. 1-(tert-Butyl) 2-Ethyl 4-bromo-1H-pyrrole-1,2-dicarboxylate (22) [59], tert-Butyl $(S)$ - and (R)-[1-hydroxy-4-(methylthio)butan-2-yl]carbamate $[(S)-\mathbf{1 0}$ and $(R)-10][50,51]$ methyl $1 H$-pyrrole-2-carboxylate (24c) [63] and 4-[3-(4-fluorophenyl)-5-isopropylisoxazol-4-yl]pyridin-2-amine (30) [34] were prepared following literature procedures.

Chiral and achiral high-performance liquid chromatography (HPLC) analyses were performed on an Agilent 1100 Quaternary pump HPLC system (Waldbronn, Germany) with a diode array detector (200-400 nm), employing columns as indicated in the supporting information. Injection volumes were typically $10 \mu \mathrm{L}\left(1-2 \mathrm{mg} \cdot \mathrm{mL}^{-1}\right)$ and data was processed with Agilent Cerity System software. 
LC-MS was performed with a Bruker Esquire $\sim$ LC ion trap mass spectrometer (Bremen, Germany) in the positive ion mode (dry gas $9 \mathrm{~L} / \mathrm{min}$, nebulizer $35 \mathrm{psi}$, drying temperature $350{ }^{\circ} \mathrm{C}$ ) after chromatographic separation using an Agilent 1100 HPLC system (Waldbronn, Germany) with a RP-8 column (Agilent Eclipse XDB-C8, $150 \times 4.6 \mathrm{~mm}, 5 \mu \mathrm{m}$ ) and a $0.1 \%$ acetic acid/acetonitrile gradient.

\subsubsection{Syntheses}

(S)-tert-Butyl 1-(tert-butyldiphenylsilyloxy)-4-(methylthio)-butan-2-ylcarbamate [(S)-11]. To a solution of alcohol $(S)-10(17.4 \mathrm{~g}, 73.9 \mathrm{mmol})$ in dry DMF $(110 \mathrm{~mL})$ was added imidazole $(12.6 \mathrm{~g}, 185 \mathrm{mmol})$ and TBDPSCl (21.2 $\mathrm{mL}, 81.5 \mathrm{mmol})$, and the resulting reaction mixture was stirred at room temperature overnight. Water $(500 \mathrm{~mL})$ was added and the mixture extracted with ethyl acetate $(3 \times)$. The combined organic layers were washed with water $(2 \times)$ and brine, dried over $\mathrm{MgSO}_{4}$ and concentrated. The crude product was purified by flash column chromatography (silica gel, ethyl acetate/petroleum ether 1:19, $1: 9$ and 1:6) to yield the desired product $(S)-11$ quantitatively as a colorless oil. $[\alpha]_{\mathrm{D}}^{23}=-11.7(c 1.20$, $\left.\mathrm{CHCl}_{3}\right) ;{ }^{1} \mathrm{H}$ NMR $\left(500 \mathrm{MHz}, \mathrm{CDCl}_{3}\right) \delta 7.66-7.61(\mathrm{~m}, 4 \mathrm{H}), 7.45-7.34(\mathrm{~m}, 6 \mathrm{H}), 4.76-4.64(\mathrm{~m}, 1 \mathrm{H}), 3.81-3.65$ $(\mathrm{m}, 2 \mathrm{H}), 3.61(\mathrm{dd}, 10.0,3.0 \mathrm{~Hz}, 1 \mathrm{H}), 2.53-2.42(\mathrm{~m}, 2 \mathrm{H}), 2.07(\mathrm{~s}, 3 \mathrm{H}), 1.90-1.72(\mathrm{~m}, 2 \mathrm{H}), 1.44(\mathrm{~s}, 9 \mathrm{H})$, 1.07 (s, 9H); ${ }^{13} \mathrm{C}$ NMR $\left(125 \mathrm{MHz} \mathrm{CDCl}_{3}\right) \delta 155.53,135.58,135.56,133.26,133.20,129.82,129.79,127.77$, 79.22, 65.62, 51.38, 31.75, 30.77, 28.41, 26.91, 19.32, 15.52. HRMS (ES+) $m / z$ calcd for $\mathrm{C}_{26} \mathrm{H}_{39} \mathrm{NO}_{3} \mathrm{SSiNa}^{+}$ 496.2312, found 496.2307.

tert-Butyl \{(2S)-1-[(tert-butyldiphenylsilyl)oxy]-4-(methylsulfinyl)butan-2-yl\} carbamate [(S)-12]. To a solution of $(S)-\mathbf{1 1}(1.03 \mathrm{~g}, 2.17 \mathrm{mmol})$ in dichloromethane $(46 \mathrm{~mL})$ was added a solution of $m$-CPBA $(50-55 \% ; 750 \mathrm{mg}, 2.17 \mathrm{mmol})$ in dichloromethane $(4.5 \mathrm{~mL})$ dropwise at $-20^{\circ} \mathrm{C}$. After being stirred for $1 \mathrm{~h}$ at this temperature the reaction mixture was allowed to warm to $0{ }^{\circ} \mathrm{C}$ and was quenched with saturated sodium carbonate solution. The organic layer was separated, washed another two times with saturated sodium carbonate solution, dried over $\mathrm{MgSO}_{4}$ and concentrated. The residue was purified by flash column chromatography (silica gel, ethyl acetate/petroleum ether 3:7 and 1:0) to give $737 \mathrm{mg}$ $(69 \%)$ of the sulfoxide (S)-12 as a light-yellow heavy oil that solidified on standing. In addition, $211 \mathrm{mg}$ $(19 \%)$ of the sulfone $(S)-\mathbf{1 3}$ were isolated as a colorless solid.

tert-Butyl $\left\{(2 S)-1-\left[(\text { tert-butyldiphenylsilyl)oxy]-4-(methylsulfinyl)butan-2-yl }\}_{\text {carbamate }}[(S)-\mathbf{1 2}] .[\alpha]_{\mathrm{D}}^{20}=\right.\right.$ -9.3 (c 0.55, $\left.\mathrm{CHCl}_{3}\right) ;{ }^{1} \mathrm{H}$ NMR $\left(500 \mathrm{MHz}, \mathrm{CDCl}_{3}\right) \delta 7.65-7.61(\mathrm{~m}, 4 \mathrm{H}), 7.46-7.36(\mathrm{~m}, 6 \mathrm{H}), 4.86-4.71(\mathrm{~m}$, $1 \mathrm{H}, \mathrm{NH}) 3.85-3.61(\mathrm{~m}, 3 \mathrm{H}), 2.78-2.64(\mathrm{~m}, 2 \mathrm{H}), 2.54(\mathrm{~s}, 1.5 \mathrm{H}), 2.53(\mathrm{~s}, 1.5 \mathrm{H}), 2.07-1.90(\mathrm{~m}, 2 \mathrm{H}), 1.44(\mathrm{~s}$, 9H), 1.07 (s, 9H); $\left.{ }^{13} \mathrm{C} \mathrm{NMR} \mathrm{(125} \mathrm{MHz,} \mathrm{CDCl}_{3}\right) \delta 155.72,155.66,135.55,135.53,133.00,132.93,129.91$, $129.89,127.83,79.52,65.85,65.81,51.70,51.40,51.01,38.70,38.65,28.36,26.90,25.73,25.22,19.28$; HRMS (ES+) $m / z$ calcd for $\mathrm{C}_{26} \mathrm{H}_{39} \mathrm{NO}_{4} \mathrm{SSiNa}^{+}$512.2261, found 512.2261.

tert-Butyl (S)-\{1-[(tert-butyldiphenylsilyl)oxy]-4-(methylsulfonyl)butan-2-yl\}carbamate [(S)-13]. m.p.: $95^{\circ} \mathrm{C} ;[\alpha]_{\mathrm{D}}^{20}=-6.3\left(\mathrm{c} 1.115, \mathrm{CHCl}_{3}\right) ;{ }^{1} \mathrm{H} \mathrm{NMR}\left(500 \mathrm{MHz}, \mathrm{CDCl}_{3}\right) \delta 7.65-7.60(\mathrm{~m}, 4 \mathrm{H}), 7.47-7.37(\mathrm{~m}, 6 \mathrm{H})$, $4.75(\mathrm{~d}, 7.4 \mathrm{~Hz}, 1 \mathrm{H}), 3.80-3.67$ overlapping signals $(\mathrm{m}, 1 \mathrm{H}$ and 3.71 , dd, 10.4, $4.1 \mathrm{~Hz}, 1 \mathrm{H}), 3.63(\mathrm{dd}, 10.3$; $3.8 \mathrm{~Hz}, 1 \mathrm{H}), 3.10-2.99(\mathrm{~m}, 2 \mathrm{H}), 2.88(\mathrm{~s}, 3 \mathrm{H}), 2.11-1.97(\mathrm{~m}, 2 \mathrm{H}), 1.44(\mathrm{~s}, 9 \mathrm{H}), 1.08(\mathrm{~s}, 9 \mathrm{H}) ;{ }^{13} \mathrm{C} \mathrm{NMR}(125$ $\left.\mathrm{MHz}_{2} \mathrm{CDCl}_{3}\right) \delta 155.64,135.57,135.53,132.87,132.78,130.00,129.96,127.90,127.87,79.78,65.71,51.99$, $50.87,40.69,28.34,26.90,25.19,19.27$; HRMS (ES+) $m / z$ calcd for $\mathrm{C}_{26} \mathrm{H}_{39} \mathrm{NO}_{5} \mathrm{SSiNa}^{+} 528.2210$, found 528.2202 .

tert-Butyl (S)-\{1-[(tert-butyldiphenylsilyl)oxy]but-3-en-2-yl\}carbamate [(S)-14]. A mixture of sulfoxide (S)-12 (29.9 g, $61.1 \mathrm{mmol})$ and calcium carbonate (14.7 g, $147 \mathrm{mmol})$ in 1,2-dichlorobenzene (165 mL) was heated under reflux for $7 \mathrm{~h}$ (TLC analysis). After being cooled to room temperature the mixture was filtered through a pad of celite and the celite was additionally washed with ethyl acetate. The filtrate was concentrated to small volume and the residue was purified by flash column chromatography to afford $18.4 \mathrm{~g}(71 \%)$ of vinylglycinol $(S)-14$ as a light-yellow oil. $[\alpha]_{\mathrm{D}}^{22}=-29.5\left(c 1.18, \mathrm{CHCl}_{3}\right)$, Ref. [56]: $[\alpha]_{\mathrm{D}}^{25}=-27.7\left(\mathrm{c} 1.02, \mathrm{CHCl}_{3}\right) ;{ }^{1} \mathrm{H}$ NMR $\left(300 \mathrm{MHz}, \mathrm{CHCl}_{3}\right) \delta 7.67-7.61(\mathrm{~m}, 4 \mathrm{H}), 7.46-7.33(\mathrm{~m}, 6 \mathrm{H}), 5.84$ (ddd, 17.2, 10.4, 5.4 Hz, 1H), $5.22(\mathrm{dt}, 17.3,1.4 \mathrm{~Hz}, 1 \mathrm{H}), 5.16(\mathrm{dt}, 10.5,1.4 \mathrm{~Hz}, 1 \mathrm{H}), 4.86-4.73(\mathrm{~m}, 1 \mathrm{H})$, 4.30-4.16 (m, 1H), $3.74(\mathrm{dd}, 10.1,4.4 \mathrm{~Hz}, 1 \mathrm{H}), 3.64(\mathrm{dd}, 10.1,4.6 \mathrm{~Hz}, 1 \mathrm{H}), 1.45(\mathrm{~s}, 9 \mathrm{H}), 1.06(\mathrm{~s}, 9 \mathrm{H}) ;{ }^{13} \mathrm{C}$ 
NMR $\left(75 \mathrm{MHz}, \mathrm{CHCl}_{3}\right) \delta 155.42,136.48,135.62,135.56,133.30,133.19,129.77,127.72,115.63,79.35$, 66.05, 54.41, 28.41, 26.84, 19.31; HRMS (ESI) $\mathrm{m} / z$ calcd for $\mathrm{C}_{25} \mathrm{H}_{35} \mathrm{NO}_{3} \mathrm{SiNa}^{+} 448.2278$, found 488.2589. tert-Butyl (S)-allyl\{1-[(tert-butyldiphenylsilyl)oxy]but-3-en-2-yl\}carbamate [(S)-15]. To a solution of vinylglycinol (S)-14 (18.3 g, $43.0 \mathrm{mmol})$ and allyl bromide $(6.78 \mathrm{~mL}, 78.4 \mathrm{mmol})$ in dry DMF $(140 \mathrm{~mL})$ was added sodium hydride $(60 \%$, in mineral oil; $2.35 \mathrm{~g}, 58.8 \mathrm{mmol})$ portionwise at room temperature followed by a catalytic amount of tetra-n-butyl ammonium iodide. The ice bath was removed, and the reaction was stirred at room temperature overnight. Another portion of allyl bromide $(6.78 \mathrm{~mL}, 78.4$ $\mathrm{mmol})$ and sodium hydride $(60 \%$, in mineral oil; $2.35 \mathrm{~g}$, $58.8 \mathrm{mmol})$ was added at room temperature and the mixture was again stirred overnight. After a third addition of allyl bromide ( $3.00 \mathrm{~mL}, 34.7 \mathrm{mmol})$ and sodium hydride $(60 \%$, in mineral oil; $1.00 \mathrm{~g}, 25.0 \mathrm{mmol})$ and stirring at room temperature overnight water was added carefully and the mixture was extracted with ethyl acetate $(3 \times)$. The organic phases were combined, washed with water $(2 \times)$ and brine, dried over $\mathrm{MgSO}_{4}$ and concentrated. The obtained crude residue was purified by flash column chromatography (silica gel, ethyl acetate/petroleum ether 1:30) to yield $15.8 \mathrm{~g}(79 \%)$ of $N$-allylvinylglycinol $(S)-\mathbf{1 5}$ as a colorless oil. $[\alpha]_{\mathrm{D}}^{23}=+0.82\left(c 0.90, \mathrm{CHCl}_{3}\right)$. ${ }^{1} \mathrm{H}$ NMR $\left(500 \mathrm{MHz}, d_{6}\right.$-DMSO, rotamers) $\delta 7.64-7.59(\mathrm{~m}, 4 \mathrm{H}), 7.50-7.41(\mathrm{~m}, 6 \mathrm{H}), 5.87-5.74(\mathrm{~m}, 2 \mathrm{H}), 5.15$ (dt, 10.6, $1.4 \mathrm{~Hz}, 1 \mathrm{H}), 5.13-5.06$ (overlapping signals: $\mathrm{m}, 1 \mathrm{H}$ and 5.10, $\mathrm{dt}, 17.4,1.4 \mathrm{~Hz}, 1 \mathrm{H}$ ), 5.05-5.01 $(\mathrm{m}, 1 \mathrm{H}), 4.70-4.55(\mathrm{~m}, 0.5 \mathrm{H}), 4.36-4.20(\mathrm{~m}, 0.4 \mathrm{H}), 3.87-3.64$ (overlapping signals: 3.81 , dd, 10.2, $8.3 \mathrm{~Hz}$, $1 \mathrm{H}$ and $\mathrm{m}, 3 \mathrm{H}), 1.37\left(\mathrm{~s}_{\mathrm{br}}, 9 \mathrm{H}\right), 0.99(\mathrm{~s}, 9 \mathrm{H}) ;{ }^{13} \mathrm{C}$ NMR (125 MHz, $d_{6}$-DMSO, rotamers) $\delta 154.48,135.98$, $135.52,134.93,134.46,132.75,129.79,127.74,117.28,115.69,115.27,78.69,63.81,63.32,60.69,59.29,47.71$, 46.84, 27.90, 26.42, 18.63; ${ }^{1} \mathrm{H}$ NMR $\left(500 \mathrm{MHz}, d_{6}\right.$-DMSO, $\left.100{ }^{\circ} \mathrm{C}\right) \delta 7.65-7.61(\mathrm{~m}, 4 \mathrm{H}), 7.48-7.39(\mathrm{~m}, 6 \mathrm{H})$, 5.87 (ddd, 17.3, 10.8, $6.4 \mathrm{~Hz}, 1 \mathrm{H}), 5.80$ (ddt, 17.2, 10.5, $5.7 \mathrm{~Hz}, 1 \mathrm{H}), 5.17-5.07$ (overlapping signals: 5.15, $\mathrm{dt}, 10.6,1.5 \mathrm{~Hz}, 1 \mathrm{H}$ and, $\mathrm{m}, 2 \mathrm{H}), 5.02(\mathrm{ddd}, 10.3,3.0,1.5 \mathrm{~Hz}, 1 \mathrm{H}), 4.47-4.39(\mathrm{~m}, 1 \mathrm{H}), 3.88(\mathrm{dd}, 10.3$, $7.6 \mathrm{~Hz}, 1 \mathrm{H}$ ), 3.85-3.77 (overlapping signals: 3.82, ddt, 16.1, 5.6, $1.4 \mathrm{~Hz}, 1 \mathrm{H}$ and 3.79, dd, 10.3, $6.1 \mathrm{~Hz}$, 1H), 3.74 (ddt, 16.1, 5.8, $1.4 \mathrm{~Hz}, 1 \mathrm{H}), 1.38(\mathrm{~s}, 9 \mathrm{H}), 1.03(\mathrm{~s}, 9 \mathrm{H}),{ }^{13} \mathrm{C} \mathrm{NMR}\left(125 \mathrm{MHz}, d_{6}-\mathrm{DMSO}, 100{ }^{\circ} \mathrm{C}\right)$ $\delta$ 154.04, 135.34, 134.52, 134.47, 132.71, 129.15, 127.12, 116.41, 114.87, 78.38, 63.62, 59.94, 47.19, 27.52, 26.12, 18.20; HRMS (ESI) $m / z$ calcd for $\mathrm{C}_{28} \mathrm{H}_{39} \mathrm{NO}_{3} \mathrm{SiNa}^{+} 488.2591$, found 488.2589; Anal. calcd for $\mathrm{C}_{28} \mathrm{H}_{39} \mathrm{NO}_{3} \mathrm{Si}: \mathrm{C}, 72.21 ; \mathrm{H}, 8.44 ; \mathrm{N}, 3.01$. Found $\mathrm{C}, 72.43 ; \mathrm{H}, 8.69 ; \mathrm{N}, 3.03$.

tert-Butyl (S)-2-\{[(tert-butyldiphenylsilyl)oxy]methyl\}-2,5-dihydro-1H-pyrrole-1-carboxylate [(S)-16]. A solution of $N$-allylvinylglycinol $(S)-15(4.32 \mathrm{~g}, 9.28 \mathrm{mmol})$ in dry DCM $(40 \mathrm{~mL})$ was treated with Grubbs 1st-generation catalyst $(40.0 \mathrm{mg}, 0.049 \mathrm{mmol})$ employing the procedure described by Brackmann et al. [55] to afford $3.91 \mathrm{~g}(96 \%)$ of 3,4-dehydroprolinol (S)-16 as a colorless oil. $[\alpha]_{\mathrm{D}}^{23}=$ $-116\left(c 0.95, \mathrm{CHCl}_{3}\right)$, Ref. [58]: $[\alpha]_{\mathrm{D}}^{20}=-90.1\left(c 1.06, \mathrm{CHCl}_{3}\right)$, Ref. [57] $[\alpha]_{\mathrm{D}}^{23}=-24.6\left(c 1.00, \mathrm{CHCl}_{3}\right) ;{ }^{1} \mathrm{H}$ NMR $\left(500 \mathrm{MHz}, \mathrm{CHCl}_{3}\right.$; mixture of rotamers) $\delta 7.66-7.61(\mathrm{~m}, 4 \mathrm{H}), 7.44-7.32(\mathrm{~m}, 6 \mathrm{H}), 5.93-5.78(\mathrm{~m}, 2 \mathrm{H})$, $4.66-4.61(\mathrm{~m}, 0.4 \mathrm{H}), 4.55-4.49(\mathrm{~m}, 0.6 \mathrm{H}), 4.25(\mathrm{dd}, 15.4,1.5 \mathrm{~Hz}, 0.6 \mathrm{H}), 4.17(\mathrm{dd}, 15.4,1.2 \mathrm{~Hz}, 0.4 \mathrm{H}), 4.09$ $(\mathrm{d}, 5.3 \mathrm{~Hz}, 0.6 \mathrm{H}), 4.06(\mathrm{~d}, 5.3 \mathrm{~Hz}, 0.4 \mathrm{H}), 3.99(\mathrm{dd}, 9.8,5.0 \mathrm{~Hz}, 0.4 \mathrm{H}), 3.88(\mathrm{dd}, 9.5,3.1 \mathrm{~Hz}, 0.6 \mathrm{H}), 3.82(\mathrm{dd}$, 9.8, $2.1 \mathrm{~Hz}, 0.4 \mathrm{H}), 3.67(\mathrm{dd}, 9.4,6.5 \mathrm{~Hz}, 0.6 \mathrm{H}), 1.48(\mathrm{~s}, 3.4 \mathrm{H}), 1.35(\mathrm{~s}, 5.2 \mathrm{H}), 1.03(\mathrm{~s}, 5 \mathrm{H}), 1.02(\mathrm{~s}, 4 \mathrm{H}) ;{ }^{13} \mathrm{C}$ NMR (125 MHz, $\mathrm{CHCl}_{3}$; mixture of rotamers) $\delta 154.13,154.08,135.55,133.90,133.80,133.68,133.57$, $129.64,129.52,128.85,128.75,127.67,127.59,126.29,79.46,79.17,65.57,65.42,65.14,63.74,54.27,53.96$, 28.57, 28.45, 26.78, 19.35, 19.28; HRMS (ESI) $m / z$ calcd for $\mathrm{C}_{26} \mathrm{H}_{35} \mathrm{NO}_{3} \mathrm{SiNa}^{+} 460.2278$, found 460.2289 .

tert-Butyl (2R,3R,4S)-2-\{[(tert-butyldiphenylsilyl)oxy]methyl\}-3,4-dihydroxypyrrolidine-1-carboxylate [(-)-17]. Following the procedure described by Murruzzu and Riera [57] 3,4-dehydroprolinol (S)-16 $(461 \mathrm{mg}, 1.05 \mathrm{mmol})$ was dissolved in a 3:1 mixture of acetone $(14 \mathrm{~mL})$ and water $(4.7 \mathrm{~mL})$ and reacted with osmium tetroxide $(7 \mathrm{mg}, 0.028 \mathrm{mmol})$ in the presence of $\mathrm{N}$-methylmorpholine $\mathrm{N}$-oxide $(325 \mathrm{mg}$, $2.40 \mathrm{mmol}$ ) at room temperature overnight. The crude reaction product was purified by automated column chromatography (silica gel, ethyl acetate/petroleum ether 2-50\%) to afford $477 \mathrm{mg}(96 \%)$ of $(-)-\mathbf{1 7}$ as a colorless oil. $[\alpha]_{\mathrm{D}}^{20}=-27.3(c 0.59, \mathrm{MeOH})$, Ref. [58]: $[\alpha]_{\mathrm{D}}^{20}=-30.5(c 1.01, \mathrm{MeOH}) ;{ }^{1} \mathrm{H} \mathrm{NMR}$ $\left(500 \mathrm{MHz}, \mathrm{CD}_{3} \mathrm{OD}\right.$; mixture of rotamers) $\delta 7.68-7.61(\mathrm{~m}, 4 \mathrm{H}), 7.47-7.36(\mathrm{~m}, 6 \mathrm{H}), 4.41-4.34(\mathrm{~m}, 1.6 \mathrm{H})$, $4.30(\mathrm{t}, 3.8 \mathrm{~Hz}, 0.4 \mathrm{H}), 4.07(\mathrm{dd}, 10.5,3.7, \mathrm{~Hz}, 0.4 \mathrm{H}), 3.88(\mathrm{dd}, 10.5,4.3 \mathrm{~Hz}, 0.6 \mathrm{H}), 3.77(\mathrm{dd}, 10.5,2.0 \mathrm{~Hz}$, $0.6 \mathrm{H}), 3.73(\mathrm{dd}, 10.5,1.4 \mathrm{~Hz}, 0.4 \mathrm{H}), 3.70-3.63(\mathrm{~m}, 1 \mathrm{H}), 3.55(\mathrm{dd}, 11.1,6.1 \mathrm{~Hz}, 0.4 \mathrm{H}), 3.51(\mathrm{dd}, 11.2,6.2$ $\mathrm{Hz}, 0.6 \mathrm{H}), 3.44-3.38(\mathrm{~m}, 1 \mathrm{H}), 1.48(\mathrm{~s}, 4 \mathrm{H}), 1.29(\mathrm{~s}, 5 \mathrm{H}), 1.05(\mathrm{~s}, 5 \mathrm{H}), 1.04(\mathrm{~s}, 4 \mathrm{H}) ;{ }^{13} \mathrm{C} \mathrm{NMR}(125 \mathrm{MHz}$, 
$\mathrm{CD}_{3} \mathrm{OD}$; mixture of rotamers) $\delta 156.53,156.49,136.71,136.66,134.57,134.46,134.36,131.05,130.98$, $130.95,128.99,128.95,128.92,128.86,81.25,80.97,74.99,74.50,71.33,70.84,66.35,66.08,63.81,62.94$, 52.81, 52.17, 28.89, 28.71, 27.39, 20.14, 20.10; HRMS (ESI) $m / z$ calcd for $\mathrm{C}_{26} \mathrm{H}_{37} \mathrm{NO}_{5} \mathrm{SiNa}^{+} 494.2333$, found 494.2345 .

tert-Butyl(3aR,4R,6aS)-4-\{[(tert-butyldiphenylsilyl)oxy]methyl\}-2,2-dimethyltetrahydro-5H-

[1,3] dioxolo[4,5-c]pyrrole-5-carboxylate [(-)-18]. To a solution of compound (-)-17 (334 $\mathrm{mg}, 0.708 \mathrm{mmol})$ and 2,2-dimethoxypropane $(0.20 \mathrm{~mL}, 1.63 \mathrm{mmol})$ in acetone $(17 \mathrm{~mL})$ was added a catalytic amount of p-toluenesulfonic acid monohydrate $(26.6 \mathrm{mg}, 0.140 \mathrm{mmol})$. After being stirred at room temperature overnight saturated bicarbonate solution $(10 \mathrm{~mL})$ and water are added, and the mixture was extracted with ethyl acetate $(3 \times)$. The combined organic phases washed with brine, dried over $\mathrm{MgSO}_{4}$ and concentrated. The residue was purified by automated column chromatography (silica gel, ethyl acetate/petroleum ether $2-5 \%)$ to yield $328 \mathrm{mg}(91 \%)$ of $(-)-\mathbf{1 8}$ as a colorless oil that solidified on standing. $[\alpha]_{\mathrm{D}}^{20}=-47.9\left(c\right.$ 0.85, $\left.\mathrm{CHCl}_{3}\right)$, Ref. [57]: $[\alpha]_{\mathrm{D}}^{23}=-36.1\left(c\right.$ 1.05, $\left.\mathrm{CHCl}_{3}\right) ;{ }^{1} \mathrm{H} \mathrm{NMR}(500 \mathrm{MHz}$, $\mathrm{CHCl}_{3}$; mixture of rotamers) $\delta 7.66-7.56(\mathrm{~m}, 4 \mathrm{H}), 7.46-7.34(6 \mathrm{H}), 4.84-4.76$ (overlapping signals: $\mathrm{m}, 1 \mathrm{H}$ and $4.78, \mathrm{~d}, 6.0 \mathrm{~Hz}, 0.6 \mathrm{H}), 4.74(\mathrm{~d}, 6.1 \mathrm{~Hz}, 0.4 \mathrm{H}), 4.14-4.11(\mathrm{~m}, 0.4 \mathrm{H}), 4.03-3.98(\mathrm{~m}, 1 \mathrm{H}), 3.84(\mathrm{dd}, 12.4$, $0.9 \mathrm{~Hz}, 0.6 \mathrm{H}), 3.77(\mathrm{dd}, 10.5,3.7 \mathrm{~Hz}, 0.6 \mathrm{H}), 3.73(\mathrm{dd}, 12.3,0.9 \mathrm{~Hz}, 0.4 \mathrm{H}), 3.70-3.63(\mathrm{~m}, 2 \mathrm{H}), 1.49(\mathrm{~s}, 4 \mathrm{H})$, $1.47(\mathrm{~s}, 1.6 \mathrm{H}), 1.46(\mathrm{~s}, 1.4 \mathrm{H}), 1.37(\mathrm{~s}, 5 \mathrm{H}), 1.35(\mathrm{~s}, 1.6 \mathrm{H}), 1.33(\mathrm{~s}, 1.4 \mathrm{H}), 1.05(\mathrm{~s}, 4 \mathrm{H}), 1.04(\mathrm{~s}, 5 \mathrm{H}) ;{ }^{13} \mathrm{C} \mathrm{NMR}$ $\left(125 \mathrm{MHz}, \mathrm{CHCl}_{3}\right.$; mixture of rotamers) $\delta 154.25,154.06,135.56,135.45,132.99,132.94,132.80,132.70$, $129.94,129.89,129.87,129.80,127.89,127.84,127.78,111.47,83.31,82.66,79.99,79.67,79.61,79.20,65.00$, $64.68,64.62,64.42,54.23,53.54,28.52,28.42,27.10,26.90,26.83,25.14,19.16,19.08$; HRMS (ESI) $\mathrm{m} / z$ calcd for $\mathrm{C}_{29} \mathrm{H}_{41} \mathrm{NO}_{5} \mathrm{SiNa}^{+} 534.2646$, found 534.2654 .

tert-Butyl(3aR,4R,6aS)-4-(hydroxymethyl)-2,2-dimethyltetrahydro-5H-[1,3]dioxolo[4,5-c]pyrrole-5carboxylate [(-)-19]. To a solution of $(-)-\mathbf{1 8}(218 \mathrm{mg}, 0.426 \mathrm{mmol})$ in THF $(3 \mathrm{~mL})$ was added a $1 \mathrm{M}$ solution of tetra- $n$-butylammonium fluoride in THF $(0.7 \mathrm{~mL}, 0.70 \mathrm{mmol})$ at room temperature. After being stirred overnight water was added and the resulting mixture extracted with ethyl acetate $(3 \times)$. The organic phases were combined, washed with brine, dried over $\mathrm{MgSO}_{4}$ and concentrated. The crude product was purified by automated column chromatography (silica gel, ethyl acetate/petroleum ether 2-20\%) to give $100 \mathrm{mg}(86 \%)$ of the desired alcohol (-)-19 as a colorless oil that solidified on standing. $[\alpha]_{\mathrm{D}}^{20}=-47.6\left(c 0.555, \mathrm{CHCl}_{3}\right)$, Ref. [57]: $[\alpha]_{\mathrm{D}}^{23}=-30.3\left(c 0.3, \mathrm{CHCl}_{3}\right) ;{ }^{1} \mathrm{H} \mathrm{NMR}(500 \mathrm{MHz}$, $\mathrm{CH}_{3} \mathrm{OD}$; mixture of rotamers) $\delta 4.78-4.73(\mathrm{~m}, 1 \mathrm{H}), 4.72-4.68(\mathrm{~m}, 1 \mathrm{H}), 3.98(\mathrm{t}, 3.7 \mathrm{~Hz}, 0.5 \mathrm{H}), 3.93(\mathrm{t}, 4.0$ $\mathrm{Hz}, 0.5 \mathrm{H}), 3.69$ (d, $11.8 \mathrm{~Hz}, 0.5 \mathrm{H}), 3.67$ (d, $11.8 \mathrm{~Hz}, 0.5 \mathrm{H}), 3.64-3.59(\mathrm{~m}, 2 \mathrm{H}), 3.50$ (dd, $12.5 \mathrm{~Hz}, 5.1 \mathrm{~Hz}$, $0.5 \mathrm{H}), 3.46(\mathrm{dd}, 12.5 \mathrm{~Hz}, 5.2 \mathrm{~Hz}, 0.5 \mathrm{H}), 1.46$ (s, 9H), $1.40(\mathrm{~s}, 3 \mathrm{H}), 1.30(\mathrm{~s}, 3 \mathrm{H}) ;{ }^{13} \mathrm{C} \mathrm{NMR}(125 \mathrm{MHz}$, $\mathrm{CD}_{3} \mathrm{OD}$; mixture of rotamers) $\delta 156.41,156.26,112.57,84.29,83.57,81.36,81.29,81.00,80.23,66.93$, $66.44,62.77,62.34,54.52,53.89,28.75,27.35,25.10$; HRMS (ESI) $m / z$ calcd for $\mathrm{C}_{13} \mathrm{H}_{23} \mathrm{NO}_{5} \mathrm{Na}^{+} 296.1468$, found 296.1480 .

tert-Butyl(3aR,4R,6aS)-2,2-dimethyl-4-\{[(methylsulfonyl)oxy]methyl\}tetrahydro-5H-[1,3]dioxo-lo[4,5c]pyrrole-5-carboxylate [(-)-20]. To a solution of (-)-19 (6.98 g, $25.5 \mathrm{mmol})$ in dry DCM (160 mL) and triethylamine $(10.7 \mathrm{~mL}, 76.7 \mathrm{mmol})$ was added methanesulfonyl chloride $(3.0 \mathrm{~mL}, 38.8 \mathrm{mmol})$ dropwise at $0{ }^{\circ} \mathrm{C}$. After being stirred for $30 \mathrm{~min}$ the reaction was poured into half concentrated bicarbonate solution. The organic phase was separated, and the aqueous layer extracted with ethyl acetate another three times. The organic phases were combined, washed with brine, dried over $\mathrm{MgSO}_{4}$ and concentrated. The recovered crude product was used in the next reaction step without further purification. An analytical sample was purified by automated column chromatography (silica gel, ethyl acetate/petroleum ether $2-50 \%)$ to yield (-)-20 as a colorless oil. $[\alpha]_{\mathrm{D}}^{20}=-50.8(c 0.62, \mathrm{MeOH})$; ${ }^{1} \mathrm{H}$ NMR (500 MHz, CD $3 \mathrm{OD}$; mixture of rotamers) $\delta 4.81-4.77(\mathrm{~m}, 1 \mathrm{H}), 4.72($ ap d, $6.1 \mathrm{~Hz}, 1 \mathrm{H}), 4.41(\mathrm{dd}$, $10.4,4.5 \mathrm{~Hz}, 0.5 \mathrm{H}), 4.35(\mathrm{dd}, 10.4,5.0 \mathrm{~Hz}, 0.5 \mathrm{H}), 4.31$ (ap dd, 10.4, 3.6 Hz, 1H), 4.20-4.14 (m, $1 \mathrm{H}), 3.74(\mathrm{~d}$, $12.6 \mathrm{~Hz}, 0.5 \mathrm{H}), 3.70(\mathrm{~d}, 12.7 \mathrm{~Hz}, 0.5 \mathrm{H}), 3.51(\mathrm{dd}, 12.7,5.1 \mathrm{~Hz}, 0.5 \mathrm{H}), 3.47$ (dd, 12.7, 5.1 Hz, 0.5H), 3.10 (s, 1.5H), 3.09 (s, 1.5H), $1.48(\mathrm{~s}, 4.5 \mathrm{H}), 1.47(\mathrm{~s}, 4.5 \mathrm{H}), 1.42(\mathrm{~s}, 3 \mathrm{H}), 1.31(\mathrm{~s}, 3 \mathrm{H}) ;{ }^{13} \mathrm{C} \mathrm{NMR}\left(125 \mathrm{MHz}, \mathrm{CD}_{3} \mathrm{OD}\right.$; mixture of rotamers) $\delta 156.19,155.81,113.06,83.86,83.08,82.08,81.83,80.76,80.00,70.00,69.81,64.45$, 64.03, 54.24, 53.46, 37.37, 37.27, 28.67, 27.30, 25.05; ${ }^{1} \mathrm{H}$ and ${ }^{13} \mathrm{C} \mathrm{NMR}$ data recorded in $\mathrm{CDCl}_{3}$ was 
consistent with that reported in reference [48,49]. HRMS (ESI) $m / z$ calcd for $\mathrm{C}_{14} \mathrm{H}_{25} \mathrm{NO}_{7} \mathrm{SNa}^{+} 374.1244$, found 374.1244 .

tert-Butyl (R)-\{1-[(tert-butyldiphenylsilyl)oxy]-4-(methylthio)butan-2-yl\}carbamate [(R)-11]. Using the same procedure as for the preparation of compound (S)-11 alcohol $(R)-\mathbf{1 0}(35.0 \mathrm{~g}, 149 \mathrm{mmol})$ was dissolved in dry DMF $(250 \mathrm{~mL})$ and reacted with TBDPSCl $(46.4 \mathrm{~mL}, 179 \mathrm{mmol})$ in the presence of imidazole $(25.3 \mathrm{~g}, 372 \mathrm{mmol})$ to yield $58.2 \mathrm{~g}(83 \%)$ of $(R)-\mathbf{1 1}$ as a colorless oil. $[\alpha]_{\mathrm{D}}^{21}=+6.8(c 1.075$, $\mathrm{CHCl}_{3}$ ); All spectroscopic data was consistent with its enantiomer (S)-11. HRMS (ES+) $\mathrm{m} / z$ calcd for $\mathrm{C}_{26} \mathrm{H}_{39} \mathrm{NO}_{3} \mathrm{SSiNa}^{+}$496.2318, found 496.2312.

tert-Butyl \{(2R)-1-[(tert-butyldiphenylsilyl)oxy]-4-(methylsulfinyl)butan-2-yl\}carbamate [(R)-12]. According to the protocol outlined for the preparation of (S)-12 sulfide (R)-11 (58.0 g, $122 \mathrm{mmol})$ dissolved in dichloromethane $(1700 \mathrm{~mL})$ was oxidized by adding dropwise a solution of $m$-CPBA $\left(50-55 \%\right.$; $20 \mathrm{~g}, 61.2 \mathrm{mmol}$ ) in dichloromethane $\left(160 \mathrm{~mL}\right.$ ) (dried over $\mathrm{MgSO}_{4}$ ) at $-40{ }^{\circ} \mathrm{C}$ to give $29.8 \mathrm{~g}$ $(50 \%)$ of the desired sulfoxide $(R)-\mathbf{1 2}$ as a colorless oil that solidified on standing. In addition, $1.77 \mathrm{~g}$ $(3 \%)$ of the sulfone $(R)-\mathbf{1 3}$ were isolated from the reaction as a colorless solid that also solidified on standing along with $26.1 \mathrm{~g}(45 \%)$ of starting material $(R)-\mathbf{1 1}$.

tert-Butyl $\left\{(2 R)-1-\left[(\right.\right.$ tert-butyldiphenylsilyl)oxy]-4-(methylsulfinyl)butan-2-yl $\}$ carbamate $[(R)-12] .[\alpha]_{\mathrm{D}}^{21}=$ +9.4 (c 1.075, $\mathrm{CHCl}_{3}$ ); All spectroscopic data was consistent with its enantiomer (S)-12. HRMS (ES+) $m / z$ calcd for $\mathrm{C}_{26} \mathrm{H}_{39} \mathrm{NO}_{4} \mathrm{SSiNa}^{+}$512.2261, found 512.2274.

tert-Butyl (R)-\{1-[(tert-butyldiphenylsilyl)oxy]-4-(methylsulfonyl)butan-2-yl\}carbamate $[(R)-13] .[\alpha]_{\mathrm{D}}^{21}=$ +6.0 (c 1.14, $\mathrm{CHCl}_{3}$ ); All spectroscopic data was consistent with its enantiomer (S)-13. HRMS (ES+) $m / z$ calcd for $\mathrm{C}_{26} \mathrm{H}_{39} \mathrm{NO}_{5} \mathrm{SSiNa}^{+}$528.2210, obsd 528.2209; Anal. calcd for $\mathrm{C}_{26} \mathrm{H}_{39} \mathrm{NO}_{5} \mathrm{SSi}: \mathrm{C}, 61.75 ; \mathrm{H}$, 7.77; N, 2.77. Found C, 62.02; H, 7.96; N, 2.68.

tert-Butyl (R)-\{1-[(tert-butyldiphenylsilyl)oxy]but-3-en-2-yl\}carbamate [(R)-14]. Employing the same procedure as for the preparation of $(S)-\mathbf{1 4}$ a mixture of sulfoxide $(R)-\mathbf{1 2}(29.6 \mathrm{~g}, 60.4 \mathrm{mmol})$ and calcium carbonate $(14.5 \mathrm{~g}, 145 \mathrm{mmol})$ in 1,2-dichlorobenzene $(165 \mathrm{~mL})$ was heated at $200{ }^{\circ} \mathrm{C}$ for $7 \mathrm{~h}$ to yield $16.8 \mathrm{~g}(65 \%)$ of vinylglycinol $(R)-\mathbf{1 4}$ as a light-yellow oil. $[\alpha]_{\mathrm{D}}^{22}=+27.0\left(c 1.03, \mathrm{CHCl}_{3}\right)$, Ref. [84]: $[\alpha]_{\mathrm{D}}^{23}=$ +25.4 ( $c$ 1.7, $\mathrm{CHCl}_{3}$ ); The spectroscopic data was consistent with that for its enantiomer (S)-14. HRMS (ESI) $m / z$ calcd for $\mathrm{C}_{25} \mathrm{H}_{35} \mathrm{NO}_{3} \mathrm{SiNa}^{+} 448.2278$, found 448.2275 .

tert-Butyl (R)-allyl\{1-[(tert-butyldiphenylsilyl)oxy]but-3-en-2-yl\}carbamate [(R)-15]. Using the same procedure as for the preparation of $\mathrm{N}$-allylvinylglycinol (S)-15 vinylglycinol (R)-14 (16.4 g, $38.5 \mathrm{mmol})$ dissolved in dry DMF $(125 \mathrm{~mL})$ was reacted with allyl bromide $(6.67 \mathrm{~mL}, 77.1 \mathrm{mmol}$; second addition after ca. $20 \mathrm{~h}: 7.0 \mathrm{~mL}, 80.9 \mathrm{mmol}$; third addition after another ca. $20 \mathrm{~h}: 7.0 \mathrm{~mL}, 80.9 \mathrm{mmol}$ ) and sodium hydride (60\% in mineral oil, $2.31 \mathrm{~g}, 57.8 \mathrm{mmol}$; second addition after ca. $20 \mathrm{~h}: 2.38 \mathrm{~g}, 59.5 \mathrm{mmol}$; third addition after another $20 \mathrm{~h}: 1.19 \mathrm{~g}, 29.8 \mathrm{mmol}$ ) in the presence of a catalytic amount of $n-\mathrm{Bu}_{4} \mathrm{NI}$ to afford $12.7 \mathrm{~g}(71 \%)$ of $\mathrm{N}$-allylvinylglycinol $(R)-\mathbf{1 5}$ as a colorless oil. In addition, $3.86 \mathrm{~g}$ of mixed starting material and product were $[\alpha]_{\mathrm{D}}^{23}=-0.76\left(c 1.07, \mathrm{CHCl}_{3}\right)$. The spectroscopic data was consistent with that reported for its enantiomer (S)-15. HRMS (ESI) $m / z$ calcd for $\mathrm{C}_{28} \mathrm{H}_{39} \mathrm{NO}_{3} \mathrm{SiNa}^{+} 488.2591$, found 488.2596; Anal. calcd for $\mathrm{C}_{28} \mathrm{H}_{39} \mathrm{NO}_{3} \mathrm{Si}: \mathrm{C}, 72.21 ; \mathrm{H}, 8.44 ; \mathrm{N}, 3.01$. Found $\mathrm{C}, 72.30 ; \mathrm{H}, 8.52 ; \mathrm{N}, 3.07$.

tert-Butyl (R)-2-\{[(tert-butyldiphenylsilyl)oxy]methyl\}-2,5-dihydro-1H-pyrrole-1-carboxylate [(R)-16]. Following the method described by Brackmann et al. [55] N-allylvinylglycinol (R)-15 (9.80 g, 21.0 $\mathrm{mmol})$ was dissolved in dry DCM $(94 \mathrm{~mL})$ and treated with Grubbs $1^{\text {st }}$-generation catalyst $(250 \mathrm{mg}$, $0.304 \mathrm{mmol}$ ) for $24 \mathrm{~h}$ at room temperature to yield $9.04 \mathrm{~g}(98 \%)$ of 3,4-dehydroprolinol (R)-16 as a colorless oil. $[\alpha]_{\mathrm{D}}^{23}=+115\left(\right.$ c 1.38, $\left.\mathrm{CHCl}_{3}\right)$. The spectroscopic data was consistent with those reported for its enantiomer (S)-16. HRMS (ES+) $m / z$ calcd for $\mathrm{C}_{26} \mathrm{H}_{35} \mathrm{NO}_{3} \mathrm{SiNa}^{+} 460.2274$, found 460.2281.

tert-Butyl (2S,3S,4R)-2-\{[(tert-butyldiphenylsilyl)oxy]methyl\}-3,4-dihydroxypyrrolidine-1-carboxylate [(+)-17]. Deploying the procedure described by Murruzzu and Riera [57] compound (R)-16 (8.85 g, $20.2 \mathrm{mmol})$ was dissolved in a 10:1 mixture of acetone $(360 \mathrm{~mL})$ and water $(36 \mathrm{~mL})$ and treated with osmium tetroxide $(59.9 \mathrm{mg}, 0.236 \mathrm{mmol})$ in the presence of $\mathrm{N}$-methylmorpholine $\mathrm{N}$-oxide $(6.01 \mathrm{~g}, 44.5$ $\mathrm{mmol})$ at room temperature overnight to yield $8.61 \mathrm{~g}(90 \%)$ of $(+)-\mathbf{1 7}$ as a colorless oil. $[\alpha]_{\mathrm{D}}^{20}=+28.6(\mathrm{c}$ 
0.545, $\mathrm{MeOH})$; All spectroscopic data was consistent with the data described for its enantiomer (-)-17. HRMS (ESI) $m / z$ calcd for $\mathrm{C}_{26} \mathrm{H}_{37} \mathrm{NO}_{5} \mathrm{SiNa}^{+} 494.2333$, found 494.2344 .

tert-Butyl(3aS,4S,6aR)-4-\{[(tert-butyldiphenylsilyl)oxy]methyl\}-2,2-dimethyltetrahydro-5H-

[1,3]dioxolo[4,5-c]pyrrole-5-carboxylate [(+)-18]. Using the same procedure as for the preparation of its enantiomer (-)-18 compound (+)-17 (8.50 g, $18.0 \mathrm{mmol})$ and 2,2-dimethoxypropane $(4.43 \mathrm{~mL}, 36.0$ $\mathrm{mmol})$ in acetone $(430 \mathrm{~mL})$ was treated with a catalytic amount of $p$-toluenesulfonic acid monohydrate $(189 \mathrm{mg}, 0.994 \mathrm{mmol})$ to give $8.76 \mathrm{~g}(95 \%)$ of $(+)-\mathbf{1 8}$ as a colorless oil that solidified on standing. $[\alpha]_{\mathrm{D}}^{20}=$ +46.1 ( c 0.92, $\mathrm{CHCl}_{3}$ ); All spectroscopic data was consistent with that of its enantiomer (-)-18. HRMS (ESI) $m / z$ calcd for $\mathrm{C}_{29} \mathrm{H}_{41} \mathrm{NO}_{5} \mathrm{SiNa}^{+}$534.2646, found 534.2652; Anal. calcd for $\mathrm{C}_{29} \mathrm{H}_{41} \mathrm{NO}_{5}$ : C, 68.07; $\mathrm{H}, 8.08 ; \mathrm{N}, 2.74$. Found C, 68.33; H, 8.20; N, 2.78 .

tert-Butyl(3aS,4S,6aR)-4-(hydroxymethyl)-2,2-dimethyltetrahydro-5H-[1,3]dioxolo[4,5-c]pyrrole-5carboxylate $[(+)-19]$. Following the same protocol as outlined for the preparation of its enantiomer (-)-19 the fully protected pyrrolidine (+)-18 $(8.67 \mathrm{~g}, 16.9 \mathrm{mmol})$ was dissolved in THF $(85 \mathrm{~mL})$ and treated with a $1 \mathrm{M}$ solution of tetra-n-butylammonium fluoride in THF $(25 \mathrm{~mL}, 25 \mathrm{mmol})$ at room temperature overnight to yield $4.65 \mathrm{~g}$ (quant.) of (+)-19 as a colorless oil that solidified on standing. $[\alpha]_{\mathrm{D}}^{20}=+46.7\left(c 0.505, \mathrm{CHCl}_{3}\right)$, Ref. [85]: $[\alpha]_{\mathrm{D}}^{28}=+29\left(c 1.0, \mathrm{CHCl}_{3}\right)$, Ref. [86]: $[\alpha]_{\mathrm{D}}^{24}=+29.4(c 1.04$, $\mathrm{CHCl}_{3}$ ); All spectroscopic data was consistent with its enantiomer (-)-19. HRMS (ESI) $\mathrm{m} / z$ calcd for $\mathrm{C}_{13} \mathrm{H}_{23} \mathrm{NO}_{5} \mathrm{Na}^{+} 296.1468$, found 296.1468; Anal. calcd for $\mathrm{C}_{13} \mathrm{H}_{23} \mathrm{NO}_{5} ; \mathrm{C}, 57.13 ; \mathrm{H}, 8.48 ; \mathrm{N}, 5.12$. Found C, 57.16; H, 8.53; N, 5.11.

tert-Butyl(3aS,4S,6aR)-2,2-dimethyl-4-\{[(methylsulfonyl)oxy]methyl\}tetrahydro-5H-[1,3]dioxolo[4,5clpyrrole-5-carboxylate [(+)-20]. Deploying the same protocol as described for the preparation of compound (-)-20 alcohol (+)-19 (2.51 g, $9.18 \mathrm{mmol})$ was dissolved in dry DCM $(40 \mathrm{~mL})$ and reacted with methanesulfonyl chloride $(1.1 \mathrm{~mL}, 14.2 \mathrm{mmol})$ in the presence of triethylamine $(3.9 \mathrm{~mL}, 28.0$ mmol) to yield $3.21 \mathrm{~g}$ (quant.) of (+)-20 as a colorless oil upon purification by automated column chromatography (silica gel, ethyl acetate/petroleum ether 2-50\%). $[\alpha]_{\mathrm{D}}^{20}=+51.5($ c $0.565, \mathrm{MeOH})$; All spectroscopic data was consistent with that reported for its enantiomer (-)-20. HRMS (ESI) $\mathrm{m} / z$ calcd for $\mathrm{C}_{14} \mathrm{H}_{25} \mathrm{NO}_{7} \mathrm{SNa}^{+} 374.1244$, found 374.1242; Anal. calcd for $\mathrm{C}_{14} \mathrm{H}_{25} \mathrm{NO}_{7} \mathrm{~S}: \mathrm{C}, 47.85 ; \mathrm{H}, 7.17 ; \mathrm{N}, 3.99$; $\mathrm{S}, 9.12$. Found $\mathrm{C}, 47.55 ; \mathrm{H}, 7.21 ; \mathrm{N}, 3.98 ; \mathrm{S}, 8.95$.

Ethyl 4-(4-methoxyphenyl)-1H-pyrrole-2-carboxylate (24a). To a solution of 1-(tert-butyl) 2-ethyl 4-bromo-1H-pyrrole-1,2-dicarboxylate 22 [59] (141 mg, $0.443 \mathrm{mmol})$ and 4-methoxyphenylboronic acid 23a (95\%; $213 \mathrm{mg}, 1.33 \mathrm{mmol})$ in DMF (7.5 mL) was added palladium-tetrakis(triphenylphosphine) $(29 \mathrm{mg}, 0.025 \mathrm{mmol})$ and a $2 \mathrm{M}$ aqueous solution of sodium carbonate $(2.25 \mathrm{~mL})$ and the resulting reaction mixture was heated at $110^{\circ} \mathrm{C}$ overnight. After being cooled to room temperature water was added and the mixture was extracted with ethyl acetate $(3 \times)$. The combined organic phases were washed with water $(2 \times)$ and brine, dried over $\mathrm{MgSO}_{4}$ and concentrated. The crude product was purified by automated flash column chromatography (silica gel, ethyl acetate/petroleum ether 2-20\%) to afford $88 \mathrm{mg}(81 \%)$ of $24 \mathrm{a}$ as a colorless solid. m.p. $136-138{ }^{\circ} \mathrm{C}$ (DSC); ${ }^{1} \mathrm{H}$ NMR $\left(500 \mathrm{MHz}, \mathrm{CDCl}_{3}\right)$ $\delta 9.22\left(\mathrm{~s}_{\mathrm{br}}, 1 \mathrm{H}\right), 7.44\left(\mathrm{AA}^{\prime} \mathrm{XX}^{\prime}, J_{\mathrm{AX}}=8.8 \mathrm{~Hz}, 2 \mathrm{H}\right), 7.15-7.12(\mathrm{~m}, 2 \mathrm{H}), 6.90\left(\mathrm{AA}^{\prime} \mathrm{XX}^{\prime}, J_{\mathrm{AX}}=8.8 \mathrm{~Hz}, 2 \mathrm{H}\right)$, 4.34 (q, $7.2 \mathrm{~Hz}, 2 \mathrm{H}), 3.82(\mathrm{~s}, 3 \mathrm{H}), 1.38(\mathrm{t}, 7.2 \mathrm{~Hz}, 3 \mathrm{H}) ;{ }^{13} \mathrm{C} \mathrm{NMR}\left(125 \mathrm{MHz}, \mathrm{CDCl}_{3}\right) \delta 161.18,158.31$, 127.35, 126.64, 126.45, 123.60, 118.73, 114.22, 112.18, 60.43, 55.32, 14.45; HRMS (ESI) $\mathrm{m} / \mathrm{z}$ calcd for $\mathrm{C}_{14} \mathrm{H}_{15} \mathrm{NO}_{3} \mathrm{Na}^{+} 268.0944$, found 268.0947 .

Ethyl 4-(4-methoxyphenyl)-1-methyl-1H-pyrrole-2-carboxylate (25a). To a solution of $\mathbf{2 4 a}$ (727 mg, $2.96 \mathrm{mmol})$ in dry DMF $(10 \mathrm{~mL})$ was added sodium hydride $(60 \%$ in mineral oil; $150 \mathrm{mg}$, $3.75 \mathrm{mmol})$ at $0{ }^{\circ} \mathrm{C}$. After $20 \mathrm{~min}$ iodomethane $(0.25 \mathrm{~mL}, 4.02 \mathrm{mmol})$ was introduced dropwise, the ice bath was removed after a further $15 \mathrm{~min}$ and the reaction was stirred for another $2.5 \mathrm{~h}$. Subsequently, water was added, and the aqueous mixture was extracted with ethyl acetate $(3 \times)$. The combined organic layers were washed with water $(2 \times)$ and brine, dried over $\mathrm{MgSO}_{4}$ and concentrated. The crude product was purified by automated flash column chromatography (silica gel, ethyl acetate/petroleum ether 2-20\%) to yield $540 \mathrm{mg}(70 \%)$ of $\mathbf{2 5 a}$ as a colorless oil that solidified on standing. ${ }^{1} \mathrm{H}$ NMR, $\left(500 \mathrm{MHz}, \mathrm{CDCl}_{3}\right)$ $\delta 7.41\left(\mathrm{AA}^{\prime} \mathrm{XX}^{\prime}, J_{\mathrm{AX}}=8.9 \mathrm{~Hz}, 2 \mathrm{H}\right), 7.15(\mathrm{~d}, 2.1 \mathrm{~Hz}, 1 \mathrm{H}), 6.98(\mathrm{~d}, 2.1 \mathrm{~Hz}, 1 \mathrm{H}), 6.89\left(\mathrm{AA}^{\prime} X^{\prime} X^{\prime}, J_{\mathrm{AX}}=8.9 \mathrm{~Hz}\right.$, 
2H), 4.30 (q, $7.1 \mathrm{~Hz}, 2 \mathrm{H}), 3.95(\mathrm{~s}, 3 \mathrm{H}), 3.81(\mathrm{~s}, 3 \mathrm{H}), 1.37(\mathrm{t}, 7.2 \mathrm{~Hz}, 3 \mathrm{H}) ;{ }^{13} \mathrm{C} \mathrm{NMR},\left(125 \mathrm{MHz}, \mathrm{CDCl}_{3}\right) \delta$ $161.34,158.18,127.38,126.25,125.57,123.82,123.31,114.50,114.21,59.89,55.33,36.89,14.48$; HRMS (ESI) $m / z$ calcd for $\mathrm{C}_{15} \mathrm{H}_{17} \mathrm{NO}_{3} \mathrm{Na}^{+} 282.1101$, found 282.1105; Anal. calcd for $\mathrm{C}_{15} \mathrm{H}_{17} \mathrm{NO}_{3}$ : $\mathrm{C}, 69.48 ; \mathrm{H}$, $6.61 ; \mathrm{N}, 5.40$. Found C, 69.45; H, 6.73; N, 5.43.

4-(4-Methoxyphenyl)-1-methyl-1H-pyrrole-2-carboxylic acid (26a). To solution of $25 \mathrm{a}$ (479 mg, 1.85 $\mathrm{mmol})$ in a 1:1 mixture of THF $(10 \mathrm{~mL})$ and methanol $(10 \mathrm{~mL})$ was added an $8 \mathrm{M}$ aqueous solution of potassium hydroxide $(3.5 \mathrm{~mL})$ and the resulting reaction mixture was heated at $50{ }^{\circ} \mathrm{C}$ overnight. After having evaporated the solvents water was added and the resulting mixture was acidified to $\mathrm{pH}$ 1 by slowly adding a $2 \mathrm{M}$ aqueous solution of hydrochloric acid (to $\mathrm{pH} 3-4$ by slowly adding a $1 \mathrm{M}$ aqueous solution of hydrochloric acid in the case of $O$-isopropylidene-protected compounds: $\mathbf{2 6} \mathbf{c}-\mathbf{f}$ ). The precipitate was extracted with ethyl acetate $(3 \times)$ and the combined organic phases were washed with brine, dried over $\mathrm{MgSO}_{4}$ and concentrated to small volume which was then treated with a small amount of petroleum ether. The ochre crystalline solid was filtered off and washed with a 3:7 mixture of ethyl acetate and petroleum ether. The collected solid was dried under high vacuum to give $344 \mathrm{mg}$ (81\%) of 26a. m.p. $203-204{ }^{\circ} \mathrm{C}$ (DSC); ${ }^{1} \mathrm{H}$ NMR, (500 MHz, $d_{6}$-DMSO) $\delta 12.22\left(\mathrm{~s}_{\mathrm{br}}, 1 \mathrm{H}\right), 7.47\left(\mathrm{AA}^{\prime} \mathrm{XX} \mathrm{X}^{\prime}\right.$, $\left.J_{\mathrm{AX}}=8.8 \mathrm{~Hz}, 2 \mathrm{H}\right), 7.42(\mathrm{~d}, 2.0 \mathrm{~Hz}, 1 \mathrm{H}), 7.09(\mathrm{~d}, 2.1 \mathrm{~Hz}, 1 \mathrm{H}), 6.90\left(\mathrm{AA}^{\prime} X^{\prime}, J_{\mathrm{AX}}=8.8 \mathrm{~Hz}, 2 \mathrm{H}\right), 3.86(\mathrm{~s}$, $3 \mathrm{H}), 3.75(\mathrm{~s}, 3 \mathrm{H}) ;{ }^{13} \mathrm{C} \mathrm{NMR},\left(125 \mathrm{MHz}, d_{6}\right.$-DMSO) $\delta 161.86,157.46,126.89,126.06,125.60,123.14,122.37$, 114.08, 113.59, 54.95, 36.33; HRMS (ESI) $m / z$ calcd for $\mathrm{C}_{13} \mathrm{H}_{13} \mathrm{NO}_{3} \mathrm{Na}^{+}$254.0788, found 254.0794; Anal. calcd for $\mathrm{C}_{13} \mathrm{H}_{13} \mathrm{NO}_{3}$ : C, 67.52; $\mathrm{H}, 5.67 ; \mathrm{N}, 6.06$. Found $\mathrm{C}, 67.51 ; \mathrm{H}, 5.59 ; \mathrm{N}, 6.21$.

1H-Benzo[d][1,2,3]triazol-1-yl 4-(4-methoxyphenyl)-1-methyl-1H-pyrrole-2-carboxylate (27a). To a solution of $26 \mathrm{a}(278 \mathrm{mg}, 1.20 \mathrm{mmol})$ and triethylamine $(0.51 \mathrm{~mL}, 3.66 \mathrm{mmol})$ in dry DMF $(10 \mathrm{~mL})$ was added HBTU ( $559 \mathrm{mg}, 1.47 \mathrm{mmol}$ ) in one portion at room temperature. After being stirred overnight water was added and the resulting mixture was extracted with ethyl acetate $(3 \times)$. The combined organic phases were washed with water $(2 \times)$ and brine, dried over $\mathrm{MgSO}_{4}$ and concentrated. The crude reaction product was purified by automated flash column chromatography (silica gel, ethyl acetate/petroleum ether 2-50\%) to yield $403 \mathrm{mg}$ of $27 \mathrm{a}(96 \%)$ as a colorless foam. ${ }^{1} \mathrm{H} \mathrm{NMR}(500 \mathrm{MHz}$, $\left.\mathrm{CDCl}_{3}\right) \delta 8.09(\mathrm{dt}, 8.5 \mathrm{~Hz}, 0.8 \mathrm{~Hz}, 1 \mathrm{H}), 7.63(\mathrm{~d}, 2.0 \mathrm{~Hz}, 1 \mathrm{H}), 7.55(\mathrm{ddd}, 8.3,6.6,0.9 \mathrm{~Hz}, 1 \mathrm{H}), 7.51$ (dt, 8.3, 1.0 Hz, 1H), $7.47\left(\mathrm{AA}^{\prime} X X^{\prime}, J_{\mathrm{AX}}=8.8 \mathrm{~Hz}, 2 \mathrm{H}\right), 7.43(\mathrm{ddd}, 8.3,6.7,1.4 \mathrm{~Hz}, 1 \mathrm{H}), 7.28(\mathrm{~d}, 1.9 \mathrm{~Hz}$, $1 \mathrm{H}), 6.95\left(\mathrm{AA}^{\prime} \mathrm{XX}^{\prime}, J_{\mathrm{AX}}=8.8 \mathrm{~Hz}, 2 \mathrm{H}\right), 3.98(\mathrm{~s}, 3 \mathrm{H}), 3.84,(\mathrm{~s}, 3 \mathrm{H}) ;{ }^{13} \mathrm{C} \mathrm{NMR}\left(125 \mathrm{MHz}, \mathrm{CDCl}_{3}\right) \delta 158.79$, 156.79, 143.57, 129.54, 129.15, 128.62, 126.55, 126.09, 125.77, 124.72, 120.54, 117.82, 116.83, 114.43, 108.49, 55.38, 36.97; HRMS (ESI) $m / z$ calcd for $\mathrm{C}_{19} \mathrm{H}_{16} \mathrm{~N}_{4} \mathrm{O}_{3} \mathrm{Na}^{+} 371.1115$, found 371.1114. Anal. calcd for $\mathrm{C}_{19} \mathrm{H}_{16} \mathrm{~N}_{4} \mathrm{O}_{3}: \mathrm{C}, 65.51 ; \mathrm{H}, 4.63 ; \mathrm{N}, 16.08$. Found C, 65.54; H, 4.50; N, 16.02 .

$\mathrm{N}-\{4-[3-(4-F l u o r o p h e n y l)-5-i s o p r o p y l i s o x a z o l-4-y l] p y r i d i n-2-y l\}-4-(4-m e t h o x y p h e n y l)-1-m e t h y l-1 H$ pyrrole-2-carboxamide (28a). To a solution of isoxazole $30(313 \mathrm{mg}, 1.05 \mathrm{mmol})$ in dry DMF $(10 \mathrm{~mL})$ was added sodium hydride ( $60 \%$ in mineral oil; $39.7 \mathrm{mg}, 0.993 \mathrm{mmol}$ ) in one portion at room temperature. After $30 \mathrm{~min}$ activated carboxylic acid $27 \mathrm{a}(195 \mathrm{mg}, 0.560 \mathrm{mmol})$ was introduced in one portion and the resulting reaction was stirred for $48 \mathrm{~h}$. Water was added and the mixture was extracted with ethyl acetate $(3 \times)$. The combined organic phases were then washed with water $(2 \times)$ and brine, dried over $\mathrm{MgSO}_{4}$ and concentrated. The crude product was purified by automated flash column chromatography (silica gel, ethyl acetate/petroleum ether 2-40\%) to afford $191 \mathrm{mg}(67 \%)$ of $28 \mathbf{a}$. Additional recrystallization from ethyl acetate/petroleum ether yielded $100 \mathrm{mg}$ of pure 28a as a colorless solid. m.p. $157-158{ }^{\circ} \mathrm{C}(\mathrm{DSC}) ;{ }^{1} \mathrm{H} \mathrm{NMR},\left(500 \mathrm{MHz}, \mathrm{CDCl}_{3}\right) \delta 8.42\left(\mathrm{~s}_{\mathrm{br}}, 1 \mathrm{H}\right), 8.27-8.23(\mathrm{~m}, 2 \mathrm{H})$, $7.43\left(\mathrm{AA}^{\prime} \mathrm{BB}^{\prime} \mathrm{X}, J_{\mathrm{AB}}=8.9, J_{\mathrm{AF}}=5.4 \mathrm{~Hz}, 2 \mathrm{H}\right), 7.40\left(\mathrm{AA}^{\prime} \mathrm{XX}^{\prime}, J_{\mathrm{AX}}=8.8,2 \mathrm{H}\right), 7.06-7.01$ (overlapping signals: $7.03, \mathrm{AA}^{\prime} \mathrm{BB}^{\prime} \mathrm{X}, J_{\mathrm{AB}}=J_{\mathrm{BF}}=8.7 \mathrm{~Hz}, 2 \mathrm{H}$ and $\left.7.02, \mathrm{~d}, 1.9 \mathrm{~Hz}, 1 \mathrm{H}\right), 7.00(\mathrm{~d}, 1.8 \mathrm{~Hz}, 1 \mathrm{H}), 6.91\left(\mathrm{AA}^{\prime} \mathrm{XX}^{\prime}, J_{\mathrm{AX}}=\right.$ $8.8 \mathrm{~Hz}, 2 \mathrm{H}), 6.74(\mathrm{dd}, 5.2,1.5 \mathrm{~Hz}, 1 \mathrm{H}), 3.99(\mathrm{~s}, 3 \mathrm{H}), 3.83(\mathrm{~s}, 3 \mathrm{H}), 3.25(\mathrm{sp}, 7.0 \mathrm{~Hz}, 1 \mathrm{H}), 1.39(\mathrm{~d}, 7.0 \mathrm{~Hz}$, $6 \mathrm{H}) ;{ }^{19} \mathrm{~F}$ NMR $\left(470 \mathrm{MHz}, \mathrm{CDCl}_{3}\right) \delta-111.12 ;{ }^{13} \mathrm{C} \mathrm{NMR},\left(125 \mathrm{MHz}, \mathrm{CDCl}_{3}\right) \delta 175.49,164.58 / 162.60(\mathrm{~d}$, 248.2 Hz), 160.04, 159.51, 158.39, 152.41, 148.03, 141.33, 130.50/130.43 (d, 8.2 Hz), 126.96, 126.27, 125.94, 125.55, 124.78, 123.98, 120.46, 115.88/115.71 (d, $21.7 \mathrm{~Hz}), 114.43,114.35,112.14,110.45,55.36,37.05$, 26.66, 21.00; HRMS (ESI) $\mathrm{m} / z$ calcd for $\mathrm{C}_{30} \mathrm{H}_{27} \mathrm{FN}_{4} \mathrm{O}_{3} \mathrm{H}^{+}$511.2140, found 511.2150; Anal. calcd for $\mathrm{C}_{30} \mathrm{H}_{27} \mathrm{FN}_{4} \mathrm{O}_{3}$ : C, 70.57; $\mathrm{H}, 5.33 ; \mathrm{N}, 10.97$. Found $\mathrm{C}, 70.60 ; \mathrm{H}, 5.41 ; \mathrm{N}, 11.03$. 
Ethyl 4-(2,4-dimethoxyphenyl)-1H-pyrrole-2-carboxylate (24b). Applying the same method as for the preparation of 24a a mixture of 1-(tert-butyl) 2-ethyl 4-bromo-1H-pyrrole-1,2-dicarboxylate 22 (1.19 g, $3.74 \mathrm{mmol})$, 2,4-dimethoxyphenylboronic acid 23b (95\%; $2.18 \mathrm{~g}, 11.4 \mathrm{mmol}$ ), palladium-tetrakis(triphenylphosphine) $(225 \mathrm{mg}, 0.195 \mathrm{mmol})$, and a $2 \mathrm{M}$ aqueous solution of sodium carbonate $(19 \mathrm{~mL})$ in DMF $(63 \mathrm{~mL})$ was heated at $110{ }^{\circ} \mathrm{C}$ overnight to yield $892 \mathrm{mg}(87 \%)$ of $\mathbf{2 4 b}$ as a colorless solid. M.p. $124-125{ }^{\circ} \mathrm{C}$ (DSC); ${ }^{1} \mathrm{H}$ NMR $\left(500 \mathrm{MHz}, \mathrm{CDCl}_{3}\right) \delta 9.13\left(\mathrm{~s}_{\mathrm{br}}, 1 \mathrm{H}\right), 7.44-7.41$ $(\mathrm{m}, 1 \mathrm{H}), 7.38(\mathrm{dd}, 2.9,1.6 \mathrm{~Hz}, 1 \mathrm{H}), 7.23(\mathrm{dd}, 2.7,1.7 \mathrm{~Hz}, 1 \mathrm{H}), 6.54-6.51(\mathrm{~m}, 2 \mathrm{H}), 4.34(\mathrm{q}, 7.1 \mathrm{~Hz}, 2 \mathrm{H})$, $3.87(\mathrm{~s}, 3 \mathrm{H}), 3.83(\mathrm{~s}, 3 \mathrm{H}), 1.37(\mathrm{t}, 7.1 \mathrm{~Hz}, 3 \mathrm{H}) ;{ }^{13} \mathrm{C} \mathrm{NMR}\left(125 \mathrm{MHz}, \mathrm{CDCl}_{3}\right) \delta 161.25,159.32,157.18$, $128.48,122.49,122.41,121.87,116.44,113.84,104.74,99.02,60.28,55.39,14.47$; HRMS (ESI) $\mathrm{m} / z$ calcd for $\mathrm{C}_{15} \mathrm{H}_{17} \mathrm{NO}_{4} \mathrm{Na}^{+}$298.1050, found 298.1049 .

Ethyl 4-(2,4-dimethoxyphenyl)-1-methyl-1H-pyrrole-2-carboxylate (25b). Employing the same procedure as described for the preparation of $25 \mathbf{a}$ pyrrole $24 \mathbf{b}(790 \mathrm{mg}, 2.87 \mathrm{mmol})$ dissolved in dry DMF $(10 \mathrm{~mL})$ was treated with sodium hydride $(60 \%$ in mineral oil; $140 \mathrm{mg}, 3.50 \mathrm{mmol})$ and iodomethane $(0.23 \mathrm{~mL}, 3.70 \mathrm{mmol})$ to yield $758 \mathrm{mg}(91 \%)$ of $\mathbf{2 5 b}$ as a colorless oil. ${ }^{1} \mathrm{H}$ NMR $(500 \mathrm{MHz}$, $\left.\mathrm{CDCl}_{3}\right)$ 8 7.42-7.38 (m, 1H), 7.24-7.21 (m, 2H), 6.54-6.49 (m, 2H), $4.30(\mathrm{q}, 7.1 \mathrm{~Hz}, 2 \mathrm{H}), 3.94(\mathrm{~s}, 3 \mathrm{H}), 3.87$ $(\mathrm{s}, 3 \mathrm{H}), 3.82(\mathrm{~s}, 3 \mathrm{H}), 1.36(\mathrm{t}, 7.1 \mathrm{~Hz}, 3 \mathrm{H}) ;{ }^{13} \mathrm{C}$ NMR $\left(125 \mathrm{MHz}, \mathrm{CDCl}_{3}\right) \delta 161.47,159.19,157.11,128.77$, $128.32,122.25,119.63,116.48,116.20,104.75,98.99,59.76,55.41,36.82,14.51 ;$ HRMS (ESI) $\mathrm{m} / z$ calcd for $\mathrm{C}_{16} \mathrm{H}_{19} \mathrm{NO}_{4} \mathrm{Na}^{+} 312.1206$, found 312.1205; Anal. calcd for $\mathrm{C}_{16} \mathrm{H}_{19} \mathrm{NO}_{4}$ : C, 66.42; $\mathrm{H}, 6.62 ; \mathrm{N}, 4.84$. Found C, 66.59; H, 6.53; N, 4.90 .

4-(2,4-Dimethoxyphenyl)-1-methyl-1H-pyrrole-2-carboxylic acid (26b). Following the procedure described for the preparation of $\mathbf{2 6 a}$ ethyl ester $\mathbf{2 5 b}(522 \mathrm{mg}, 1.80 \mathrm{mmol})$ dissolved in a 1:1 mixture of THF and methanol $(10 \mathrm{~mL} / 10 \mathrm{~mL})$ was treated with an $8 \mathrm{M}$ aqueous potassium hydroxide solution $(2.5 \mathrm{~mL})$ to afford $294 \mathrm{mg}(62 \%)$ of $\mathbf{2 6 b}$ as a crystalline, ochre solid. m.p. $175-176{ }^{\circ} \mathrm{C}$ (DSC); ${ }^{1} \mathrm{H}$ NMR $\left(500 \mathrm{MHz}, d_{6}\right.$-DMSO) $\delta 7.42(\mathrm{~d}, 8.5 \mathrm{~Hz}, 1 \mathrm{H}), 7.41(\mathrm{~d}, 2.0 \mathrm{~Hz}, 1 \mathrm{H}), 7.13(\mathrm{~d}, 2.0 \mathrm{~Hz}, 1 \mathrm{H}), 6.60(\mathrm{~d}, 2.5 \mathrm{~Hz}$, $1 \mathrm{H}), 6.53(\mathrm{dd}, 8.5,2.5 \mathrm{~Hz}, 1 \mathrm{H}), 3.86(\mathrm{~s}, 3 \mathrm{H}), 3.84(\mathrm{~s}, 3 \mathrm{H}), 3.77(\mathrm{~s}, 3 \mathrm{H}) ;{ }^{13} \mathrm{C}$ NMR $\left(125 \mathrm{MHz}, d_{6}-\mathrm{DMSO}\right) \delta$ $161.96,158.57,156.60,128.46,127.55,121.99,118.68,115.59,105.18,98.77,55.28,55.08,36.22$; HRMS (ESI) $m / z$ calcd for $\mathrm{C}_{14} \mathrm{H}_{15} \mathrm{NO}_{4} \mathrm{Na}^{+} 284.0893$, found 284.0898; Anal. calcd for $\mathrm{C}_{14} \mathrm{H}_{15} \mathrm{NO}_{4}$ : $\mathrm{C}, 64.36 ; \mathrm{H}$, $5.79 ; \mathrm{N}, 5.36$. Found $\mathrm{C}, 64.46 ; \mathrm{H}, 5.68 ; \mathrm{N}, 5.38$.

1H-Benzo[d][1,2,3]triazol-1-yl 4-(2,4-dimethoxyphenyl)-1-methyl-1H-pyrrole-2-carboxylate (27b). Carboxylic acid 26b (225 mg, $0.861 \mathrm{mmol})$ in dry DMF $(8 \mathrm{~mL})$ was reacted with HBTU $(398 \mathrm{mg}, 1.05$ $\mathrm{mmol})$ in the presence of triethylamine $(0.40 \mathrm{~mL}, 2.87 \mathrm{mmol})$ according to the procedure outlined for the preparation of compound $\mathbf{2 7 a}$ to give $293 \mathrm{mg}(90 \%)$ of $\mathbf{2 7 b}$ as a colorless foam. ${ }^{1} \mathrm{H} \mathrm{NMR}(500 \mathrm{MHz}$, $\left.\mathrm{CDCl}_{3}\right) \delta 8.09(\mathrm{~d}, 8.5 \mathrm{~Hz}, 1 \mathrm{H}), 7.73(\mathrm{~d}, 1.8 \mathrm{~Hz}, 1 \mathrm{H}), 7.57-7.49(\mathrm{~m}, 3 \mathrm{H}), 7.47-7.40(\mathrm{~m}, 2 \mathrm{H}), 6.58-6.55(\mathrm{~m}$, 2H), 3.97 (s, 3H), 3.91 (s, 3H), $3.85(\mathrm{~s}, 3 \mathrm{H}) ;{ }^{13} \mathrm{C} \mathrm{NMR}\left(125 \mathrm{MHz}, \mathrm{CDCl}_{3}\right) \delta 159.86,157.26,156.87,143.57$, $132.64,129.21,128.57,128.52,124.68,121.69,120.49,119.67,115.76,115.22,108.56,104.96,99.08,55.48$, 36.86; HRMS (ESI) $m / z$ calcd for $\mathrm{C}_{20} \mathrm{H}_{18} \mathrm{~N}_{4} \mathrm{O}_{4} \mathrm{Na}^{+} 401.1220$, found 401.1229 .

4-(2,4-Dimethoxyphenyl)-N-\{4-[3-(4-fluorophenyl)-5-isopropylisoxazol-4-yl]pyridin-2-yl\}-1-methyl-1Hpyrrole-2-carboxamide (28b). Deploying the same procedure as for the preparation of compound 28a isoxazole 30 (304 mg, $1.02 \mathrm{mmol})$ was dissolved in dry DMF $(5 \mathrm{~mL})$, deprotonated with sodium hydride ( $60 \%$ in mineral oil; $36 \mathrm{mg}, 0.900 \mathrm{mmol}$ ) and subsequently reacted with a solution of $27 \mathbf{b}$ (212 $\mathrm{mg}, 0.560 \mathrm{mmol})$ in dry DMF (6 mL) to yield $172 \mathrm{mg}(57 \%)$ of $\mathbf{2 8 \mathbf { b }}$ as a colorless foam. The removal of a minor impurity required additional automated flash column chromatography on reversed phase C-18 silica gel (water/acetonitrile 10-90\%). ${ }^{1} \mathrm{H}$ NMR $\left(500 \mathrm{MHz}, \mathrm{CDCl}_{3}\right) \delta 8.51\left(\mathrm{~s}_{\mathrm{br}}, 1 \mathrm{H}\right), 8.28(\mathrm{~s}, 1 \mathrm{H}), 8.24(\mathrm{~d}$, $5.1 \mathrm{~Hz}, 1 \mathrm{H}), 7.43\left(\mathrm{AA}^{\prime} \mathrm{BB}^{\prime} \mathrm{X}, J_{\mathrm{AB}}=8.5, J_{\mathrm{AF}}=5.4 \mathrm{~Hz}, 2 \mathrm{H}\right), 7.38-7.34(\mathrm{~m}, 1 \mathrm{H}), 7.23(\mathrm{~s}, 1 \mathrm{H}), 7.14(\mathrm{~s}, 1 \mathrm{H}), 7.03$ $\left(\mathrm{AA}^{\prime} \mathrm{BB}^{\prime} \mathrm{X}, J_{\mathrm{AB}}=J_{\mathrm{BF}}=8.6 \mathrm{~Hz}, 2 \mathrm{H}\right), 6.73(\mathrm{~d}, 5.1, \mathrm{~Hz}, 1 \mathrm{H}), 6.56-6.51(\mathrm{~m}, 2 \mathrm{H}), 3.99(\mathrm{~s}, 3 \mathrm{H}), 3.89(\mathrm{~s}, 3 \mathrm{H}), 3.83$ (s, 3H), $3.26(\mathrm{sp}, 7.0 \mathrm{~Hz}, 1 \mathrm{H}), 1.39(\mathrm{~d}, 7.0 \mathrm{~Hz}, 6 \mathrm{H}) ;{ }^{19} \mathrm{~F}$ NMR $\left(470 \mathrm{MHz}, \mathrm{CDCl}_{3}\right) \delta-111.12 ;{ }^{13} \mathrm{C} \mathrm{NMR}$ $\left(125 \mathrm{MHz}, \mathrm{CDCl}_{3}\right) \delta 175.46,164.57 / 162.59$ (d, $\left.248 \mathrm{~Hz}\right), 160.03,159.68,159.42,157.16,152.58,148.07$, $141.23,130.50 / 130.43$ (d, 8.2 Hz), 128.89, 128.26, 124.80, 124.52, 120.36, 119.84, 116.05, 115.87/115.69 (d, $21.7 \mathrm{~Hz}), 114.46,112.43,112.18,104.83,99.08,55.44,36.99,26.65,20.99$; HRMS (ESI) $m / z$ calcd for 
$\mathrm{C}_{31} \mathrm{H}_{29} \mathrm{FN}_{4} \mathrm{O}_{4} \mathrm{H}^{+}$541.2246, found 541.2251. Anal. calcd for $\mathrm{C}_{31} \mathrm{H}_{29} \mathrm{FN}_{4} \mathrm{O}_{4} \cdot 0.2\left(\mathrm{CHCl}_{3}\right): \mathrm{C}, 66.39 ; \mathrm{H}, 5.21$; N, 9.93. Found C, 66.35; H, 5.22; N, 9.88.

tert-Butyl(3aR,4R,6aS)-4-\{[2-(methoxycarbonyl)-1H-pyrrol-1-yl]methyl\}-2,2-dimethyltetrahydro-5H[1,3]dioxolo[4,5-c]pyrrole-5-carboxylate (25c) To a solution of methyl 1H-pyrrole-2-carboxylate 24c [63] $(325 \mathrm{mg}, 2.60 \mathrm{mmol})$ and crude mesylate $(-)-20(1.14 \mathrm{~g}, 3.24 \mathrm{mmol})$ in dry DMF $(28 \mathrm{~mL})$ was added cesium carbonate $(2.22 \mathrm{~g}, 6.81 \mathrm{mmol})$ and a catalytic amount of tetra n-butylammonium iodide, and the resulting mixture was heated at $80{ }^{\circ} \mathrm{C}$ overnight. After being cooled to room temperature a 10:1 mixture of water and brine was added, and the aqueous mixture was extracted with ethyl acetate $(3 \times)$. The combined organic phases were washed with a 10:1 mixture of water and brine $(2 \times)$, and brine, dried over $\mathrm{MgSO}_{4}$ and concentrated. Finally, the crude reaction product was purified by automated flash column chromatography (silica gel, ethyl acetate/petroleum ether 2-40\%) to yield $909 \mathrm{mg}(92 \%)$ of $25 \mathrm{c}$ as a colorless oil that solidified on standing. $[\alpha]_{\mathrm{D}}^{20}=+4.42\left(c 0.77, \mathrm{CHCl}_{3}\right) ;{ }^{1} \mathrm{H} \mathrm{NMR}(500 \mathrm{MHz}$, $\mathrm{CDCl}_{3}$, mixture of rotamers) $\delta 6.95(\mathrm{dd}, 3.9,1.5 \mathrm{~Hz}, 1 \mathrm{H}) ; 6.88(\mathrm{~s}, 0.4 \mathrm{H}), 6.75-6.72(\mathrm{~m}, 0.6 \mathrm{H}), 6.19-6.16(\mathrm{~m}$, $0.4 \mathrm{H}), 6.15(\mathrm{t}, 3.0 \mathrm{~Hz}, 0.6 \mathrm{H}), 4.66-4.51(\mathrm{~m}, 3 \mathrm{H}), 4.49(\mathrm{dd}, 13.9,5.2 \mathrm{~Hz}, 0.4 \mathrm{H}), 4.45-4.38(\mathrm{~m}, 1 \mathrm{H}), 4.13$ $(\mathrm{dd}, 13.7,8.2 \mathrm{~Hz}, 0.6 \mathrm{H}), 3.96(\mathrm{~d}, 13.1 \mathrm{~Hz}, 0.6 \mathrm{H}), 3.80(\mathrm{~s}, 3 \mathrm{H}), 3.68(\mathrm{~d}, 12.9 \mathrm{~Hz}, 0.4 \mathrm{H}), 3.26(\mathrm{dd}, 13.1,5.0$ $\mathrm{Hz}, 0.6 \mathrm{H}), 3.17(\mathrm{dd}, 12.8,5.0 \mathrm{~Hz}, 0.4 \mathrm{H}), 1.46(\mathrm{~s}, 3.4 \mathrm{H}), 1.41(\mathrm{~s}, 3 \mathrm{H}), 1.34(\mathrm{~s}, 5.6 \mathrm{H}), 1.27(\mathrm{~s}, 2 \mathrm{H}), 1.25$ $(\mathrm{s}, 1 \mathrm{H}) ;{ }^{13} \mathrm{C} \mathrm{NMR}\left(125 \mathrm{MHz}, \mathrm{CDCl}_{3}\right.$, mixture of rotamers) $\delta 161.99,161.80,154.27,129.33,129.05$, 122.00, 121.90, 118.61, 118.48, 111.83, 111.71, 109.22, 108.92, 82.39, 81.51, 80.05, 79.97, 79.32, 78.90, 64.56, 64.02, 52.19, 51.13, 51.06, 47.49, 46.32, 28.41, 28.11, 27.02, 26.93, 25.10, 25.06; HRMS (ESI) $\mathrm{m} / \mathrm{z}$ calcd for $\mathrm{C}_{19} \mathrm{H}_{28} \mathrm{~N}_{2} \mathrm{O}_{6} \mathrm{Na}^{+}$403.1840, found 403.1844. Anal. calcd for $\mathrm{C}_{19} \mathrm{H}_{28} \mathrm{~N}_{2} \mathrm{O}_{6}$ : C, 59.99; $\mathrm{H}, 7.42 ; \mathrm{N}, 7.36$. Found $\mathrm{C}, 60.07 ; \mathrm{H}, 7.52 ; \mathrm{N}, 7.39$.

1-\{[(3aR,4R,6aS)-5-(tert-Butoxycarbonyl)-2,2-dimethyltetrahydro-4H-[1,3]dioxolo[4,5-c]pyrrol-4yl]methyl\}-1H-pyrrole-2-carboxylic acid (26c). According to the procedure described for the preparation of carboxylic acid 26a methyl ester $\mathbf{2 5 c}(752 \mathrm{mg}, 1.98 \mathrm{mmol})$ dissolved in a 2:1 mixture of THF $(12 \mathrm{~mL})$ and $\mathrm{MeOH}(6 \mathrm{~mL})$ was saponified with an aqueous $8 \mathrm{M}$ potassium hydroxide solution $82.5 \mathrm{~mL})$. The crude product was subsequently purified by automated flash column chromatography (silica gel, ethyl acetate/petroleum ether 2-70\%) to give $606 \mathrm{mg}(84 \%)$ of the desired carboxylic acid $\mathbf{2 6 \mathrm { c }}$ as a colorless oil that solidified on standing. $[\alpha]_{\mathrm{D}}^{20}=+13.2\left(c 0.50, \mathrm{CHCl}_{3}\right) ;{ }^{1} \mathrm{H} \mathrm{NMR}\left(500 \mathrm{MHz}, \mathrm{CDCl}_{3}\right.$, mixture of rotamers) $\delta 7.13-7.10(\mathrm{~m}, 1 \mathrm{H}), 6.95(\mathrm{~s}, 0.4 \mathrm{H}), 6.80-6.77(\mathrm{~s}, 0.6 \mathrm{H}), 6.24-6.21(\mathrm{~m}, 0.4 \mathrm{H}), 6.21-6.18(\mathrm{~m}, 0.6 \mathrm{H})$, $4.68-4.59(\mathrm{~m}, 2 \mathrm{H}), 4.56-4.48(\mathrm{~m}, 1.4 \mathrm{H}), 4.48-4.40(\mathrm{~m}, 1 \mathrm{H}), 4.11(\mathrm{dd}, 13.7,8.6 \mathrm{~Hz}, 0.6 \mathrm{H}), 3.99(\mathrm{~d}, 13.2 \mathrm{~Hz}$, $0.6 \mathrm{H}), 3.70(\mathrm{~d}, 12.9 \mathrm{~Hz}, 0.4 \mathrm{H}), 3.27(\mathrm{dd}, 13.2,4.9 \mathrm{~Hz}, 0.6 \mathrm{H}), 3.16(\mathrm{dd}, 12.8,4.9 \mathrm{~Hz}, 0.4 \mathrm{H}), 1.46(\mathrm{~s}, 3.4 \mathrm{H})$, $1.41(\mathrm{~s}, 3 \mathrm{H}), 1.34(\mathrm{~s}, 5.6 \mathrm{H}), 1.29(\mathrm{~s}, 2 \mathrm{H}), 1.26(\mathrm{~s}, 1 \mathrm{H}) ;{ }^{13} \mathrm{C} \mathrm{NMR}\left(125 \mathrm{MHz}, \mathrm{CDCl}_{3}\right.$, mixture of rotamers) $\delta$ 165.39, 165.23, 154.33, 130.71, 130.42, 121.13, 120.98, 120.79, 120.72, 111.92, 111.80, 109.71, 109.41, 82.35, $81.51,80.20,80.14,79.31,78.90,64.48,63.99,52.23,51.03,47.67,46.49,28.39,28.12,27.01,26.92,25.06$; HRMS (ESI) $m / z$ calcd for $\mathrm{C}_{18} \mathrm{H}_{26} \mathrm{~N}_{2} \mathrm{O}_{6} \mathrm{Na}^{+} 389.1683$, found 389.1687. Anal. calcd for $\mathrm{C}_{18} \mathrm{H}_{26} \mathrm{~N}_{2} \mathrm{O}_{6}$ : C, 59.00; H, 7.15; N, 7.65. Found C, 59.22; H, 7.19; N, 7.60.

tert-Butyl(3aR,4R,6aS)-4-\{[2-\{[(1H-benzo[d][1,2,3]triazol-1-yl)oxy]carbonyl\}-1H-pyrrol-1-yl]methyl\}-2,2dimethyltetrahydro-5H-[1,3]dioxolo[4,5-c]pyrrole-5-carboxylate (27c). Employing the same protocol as for the preparation of 27 a carboxylic acid $26 \mathrm{c}(492 \mathrm{mg}, 1.34 \mathrm{mmol})$ was dissolved in dry DMF $(15 \mathrm{~mL})$ and subsequently activated with HBTU $(625 \mathrm{mg}, 1.65 \mathrm{mmol})$ in the presence of triethylamine $(0.57$ $\mathrm{mL}, 4.09 \mathrm{mmol}$ ) to afford $648 \mathrm{mg}$ (quant.) of $27 \mathrm{c}$ as a colorless foam. $[\alpha]_{\mathrm{D}}^{20}=+31.4\left(\mathrm{c} 0.805, \mathrm{CHCl}_{3}\right)$; ${ }^{1} \mathrm{H}$ NMR $\left(500 \mathrm{MHz}, \mathrm{CDCl}_{3}\right.$, mixture of rotamers) $\delta 8.11-8.06(\mathrm{~m}, 1 \mathrm{H}), 7.59-7.47(\mathrm{~m}, 3 \mathrm{H}), 7.46-7.41$ $(\mathrm{m}, 1 \mathrm{H}), 7.21(\mathrm{~s}, 0.4 \mathrm{H}), 7.06-7.03(\mathrm{~s}, 0.6 \mathrm{H}), 6.42-6.37(\mathrm{~m}, 1 \mathrm{H}), 4.62(\mathrm{t}, 5.0 \mathrm{~Hz}, 0.6 \mathrm{H}), 4.54(\mathrm{t}, 4.8 \mathrm{~Hz}$, $0.4 \mathrm{H}), 4.51-4.37(\mathrm{~m}, 3.4 \mathrm{H}), 4.18(\mathrm{dd}, 13.5,9.3 \mathrm{~Hz}, 0.6 \mathrm{H}), 4.03(\mathrm{~d}, 13.4 \mathrm{~Hz}, 0.6 \mathrm{H}), 3.74(\mathrm{~d}, 13.1 \mathrm{~Hz}, 0.4 \mathrm{H})$, 3.22-3.13 (overlapping signals: 3.19, dd, 13.1, $4.5 \mathrm{~Hz}, 0.6 \mathrm{H}$ and $\mathrm{m}, 0.4 \mathrm{H}), 1.45(\mathrm{~s}, 4 \mathrm{H}), 1.40(\mathrm{~s}, 1 \mathrm{H}), 1.39$ $(\mathrm{s}, 2 \mathrm{H}), 1.34(\mathrm{~s}, 5 \mathrm{H}), 1.26(\mathrm{~s}, 3 \mathrm{H}) ;{ }^{13} \mathrm{C}$ NMR $\left(125 \mathrm{MHz}, \mathrm{CDCl}_{3}\right.$, mixture of rotamers) $\delta 157.23,157.05$, 154.32, 143.54, 133.20, 132.73, 129.21, 128.72, 128.59, 124.75, 122.37, 122.18, 120.56, 120.43, 116.27, 116.08, $112.07,111.00,110.67,108.71,108.49,82.35,81.53,80.41,80.28,79.39,78.98,64.90,64.02,51.93,50.84$, $48.05,47.09,28.33,28.14,26.91,26.83,24.99,24.92$; HRMS (ESI) $\mathrm{m} / \mathrm{z}$ calcd for $\mathrm{C}_{24} \mathrm{H}_{29} \mathrm{~N}_{5} \mathrm{O}_{6} \mathrm{Na}^{+} 506.2010$, found 506.2012 . 
tert-Butyl(3aR,4R,6aS)-4-\{[2-(\{4-[3-(4-fluorophenyl)-5-isopropylisoxazol-4-yl]pyridin-2-yl\}carbamoyl)1H-pyrrol-1-yl]methyl\}-2,2-dimethyltetrahydro-5H-[1,3]dioxolo[4,5-c]pyrrole-5-carboxylate (28c). Using the same procedure as for the preparation of compound 28a isoxazole 30 (376 $\mathrm{mg}, 1.26 \mathrm{mmol})$ dissolved in dry DMF (5 mL) was firstly deprotonated with sodium hydride (60\% in mineral oil; $45.0 \mathrm{mg}, 1.13$ $\mathrm{mmol}$ ) and subsequently reacted with a solution of compound $27 \mathrm{c}(339 \mathrm{mg}, 0.701 \mathrm{mmol})$ in dry DMF $(5 \mathrm{~mL})$ at room temperature over a period of $48 \mathrm{~h}$ to afford $228 \mathrm{mg}(50 \%)$ of the desired coupling product $28 \mathrm{c}$ as a colorless foam. $[\alpha]_{\mathrm{D}}^{20}=+46.8$ (c 0.66, acetone); ${ }^{1} \mathrm{H}$ NMR $\left(500 \mathrm{MHz}, \mathrm{CDCl}_{3}\right.$, mixture of rotamers) $\delta 8.37(\mathrm{~s}, 0.4 \mathrm{H}), 8.34(\mathrm{~s}, 0.6 \mathrm{H}), 8.25-8.21(\mathrm{~m}, 2 \mathrm{H}), 7.44\left(\mathrm{AA}^{\prime} \mathrm{BB}^{\prime} \mathrm{X}, J_{\mathrm{AB}}=8.6, J_{\mathrm{AF}}=5.4 \mathrm{~Hz}\right.$, $2 \mathrm{H}), 7.04\left(\mathrm{AA}^{\prime} \mathrm{BB}^{\prime} \mathrm{X}, J_{\mathrm{AB}}=J_{\mathrm{BF}}=8.6 \mathrm{~Hz}, 2 \mathrm{H}\right), 6.92(\mathrm{~s}, 0.4 \mathrm{H}), 6.84-6.78(\mathrm{~m}, 1.6 \mathrm{H}), 6.76-6.72(\mathrm{~m}, 1 \mathrm{H})$, 6.24-6.20 (m, 1H), 4.70-4.54 (m, 3H), 4.47-4.41 (m, 1.4H), $4.26(\mathrm{dd}, 13.6,8.1 \mathrm{~Hz}, 0.6 \mathrm{H}), 3.94(\mathrm{~d}, 13.1 \mathrm{~Hz}$, $0.6 \mathrm{H}), 3.71(\mathrm{~d}, 12.9 \mathrm{~Hz}, 0.4 \mathrm{H}), 3.31-3.19$ (overlapping signals: $\mathrm{m}, 1 \mathrm{H}$ and 3.26, sp, $7.0 \mathrm{~Hz}, 1 \mathrm{H}$ ), 1.44 $(\mathrm{s}, 4 \mathrm{H}), 1.40(\mathrm{~s}, 3 \mathrm{H}), 1.40(\mathrm{~d}, 6.9 \mathrm{~Hz}, 6 \mathrm{H}), 1.34(\mathrm{~s}, 5 \mathrm{H}), 1.26(\mathrm{~s}, 2 \mathrm{H}), 1.22(\mathrm{~s}, 1 \mathrm{H})$; ${ }^{19} \mathrm{~F} \mathrm{NMR}(470 \mathrm{MHz}$, $\mathrm{CDCl}_{3}$, mixture of rotamers) $\delta-110.96,-111.01 ;{ }^{13} \mathrm{C} \mathrm{NMR}\left(125 \mathrm{MHz}, \mathrm{CDCl}_{3}\right.$, mixture of rotamers $) \delta$ $175.48,164.59 / 162.61$ (d, $248.3 \mathrm{~Hz}), 160.06,159.78,159.52,154.34,154.23,152.34,152.28,148.20,141.09$, $130.55 / 130.48$ (d, 8.2 Hz), 129.27, 129.11, $124.79 / 124.76$ (d, 3.4 Hz), 124.71, 120.43, 115.89/115.72 (d, $21.6 \mathrm{~Hz}), 114.46,114.41,114.03,113.95,112.18,111.79,111.69,109.19,109.01,82.35,81.53,80.06,79.94$, $79.48,78.94,64.75,64.01,52.10,51.09,47.42,46.41,28.37,28.19,26.97,26.92,26.66,25.04,21.00$; HRMS (ESI) $m / z$ calcd for $\mathrm{C}_{35} \mathrm{H}_{40} \mathrm{FN}_{5} \mathrm{O}_{6} \mathrm{H}^{+} 646.3035$, found 646.3044 .

$(2 R, 3 R, 4 S)-2-\{[2-(\{4-[3-(4-F l u o r o p h e n y l)-5-i s o p r o p y l i s o x a z o l-4-y l] p y r i d i n-2-y l\}$ carbamoyl)-1H-pyrrol-1yl]methyl\}-3,4-dihydroxypyrrolidin-1-ium trifluoroacetate $(29 \mathrm{c})$. Trifluoroacetic acid (4.75 $\mathrm{mL})$ and water $(0.50 \mathrm{~mL})$ were added to compound $28 \mathrm{c}(163 \mathrm{mg}, 0.252 \mathrm{mmol})$ and the resulting mixture was stirred for $3 \mathrm{~h}$ at $0{ }^{\circ} \mathrm{C}$. After complete deprotection as indicated by TLC analysis the reaction was concentrated and the residue purified by automated column chromatography (silica gel, methanol/methylene chloride $0-10 \%)$ to yield $131 \mathrm{mg}(84 \%)$ of $29 \mathrm{c}$ as a colorless foam. $[\alpha]_{\mathrm{D}}^{20}=-4.5(c 0.29, \mathrm{MeOH}) ;{ }^{1} \mathrm{H}$ NMR (500 MHz, CD 3 OD) $\delta 8.35(\mathrm{dd}, 5.2,0.7 \mathrm{~Hz}, 1 \mathrm{H}), 8.08(\mathrm{dd}, \sim 1.3, \sim 0.6 \mathrm{~Hz}, 1 \mathrm{H}), 7.45\left(\mathrm{AA}^{\prime} \mathrm{BB}^{\prime} \mathrm{X}, J_{\mathrm{AB}}=\right.$ 8.9, $\left.J_{\mathrm{AF}}=5.3 \mathrm{~Hz}, 2 \mathrm{H}\right), 7.16(\mathrm{dd}, 2.6,1.7 \mathrm{~Hz}, 1 \mathrm{H}), 7.13\left(\mathrm{AA}^{\prime} \mathrm{BB}^{\prime} \mathrm{X}, J_{\mathrm{AB}}=J_{\mathrm{BF}}=8.9 \mathrm{~Hz}, 2 \mathrm{H}\right), 7.10(\mathrm{dd}, 4.1$, $1.6 \mathrm{~Hz}, 1 \mathrm{H}), 6.95(\mathrm{dd}, 5.2,1.5, \mathrm{~Hz}, 1 \mathrm{H}), 6.30(\mathrm{dd}, 4.1,2.6 \mathrm{~Hz}, 1 \mathrm{H}), 4.64(\mathrm{~d}, 6.4 \mathrm{~Hz}, 2 \mathrm{H}), 4.20(\mathrm{td}, 4.1,2.1$ $\mathrm{Hz}, 1 \mathrm{H}), 4.02$ (dd, 8.4, 4.1 Hz, 1H), 3.77 (dt, 6.5, 8.2 Hz, 1H), 3.40 (dd, 12.4, 4.2 Hz, 1H), 3.28 (sp, $7.0 \mathrm{~Hz}, 1 \mathrm{H}), 3.14(\mathrm{dd}, 12.4,2.1 \mathrm{~Hz}, 1 \mathrm{H}), 1.38(\mathrm{~d}, 7.0 \mathrm{~Hz}, 6 \mathrm{H}) ;{ }^{19} \mathrm{~F}$ NMR $\left(470 \mathrm{MHz}, \mathrm{CD}_{3} \mathrm{OD}\right) \delta-77.10$, $-112.81 ;{ }^{13} \mathrm{C}$ NMR (125 MHz, CD 3 OD) $\delta$ 176.92, 166.15/164.17 (d, 247.1 Hz), 162.97, 161.49, 153.76, 149.81, 142.26, 131.83/131.76 (d, 8.3 Hz), 131.06, 126.52, 126.19, 122.22 117.21, 117.08, 116.96/116.79 (d, 22.1 Hz), 113.62, 110.55, 75.09, 71.13, 63.96, 51.24, 48.77, 27.96, 21.25; HRMS (ESI) $\mathrm{m} / \mathrm{z}$ calcd for $\mathrm{C}_{27} \mathrm{H}_{28} \mathrm{FN}_{5} \mathrm{O}_{4} \mathrm{H}^{+}$506.2198, found 506.2203; Anal. calcd for $\mathrm{C}_{27} \mathrm{H}_{28} \mathrm{FN}_{5} \mathrm{O}_{4} \cdot 1.3\left(\mathrm{CF}_{3} \mathrm{COOH}\right): \mathrm{C}, 54.38 ; \mathrm{H}$, 4.52; N, 10.71. Found C, 54.28; H, 4.77; N, 10.85 .

tert-Butyl(3aR,4R,6aS)-4-\{[2-(ethoxycarbonyl)-4-(4-methoxyphenyl)-1H-pyrrol-1-yl]methyl\}-2,2dimethyltetrahydro-5H-[1,3]dioxolo[4,5-c]pyrrole-5-carboxylate (25d). Using the same procedure as for the preparation of compound 25c mesylate $(-)-20(2.65 \mathrm{~g}, 7.54 \mathrm{mmol})$ was dissolved in dry DMF $(40 \mathrm{~mL})$ and reacted with pyrrole $24 \mathrm{a}(1.5 \mathrm{~g}, 6.12 \mathrm{mmol})$ in the presence of cesium carbonate $(4.38 \mathrm{~g}, 13.4 \mathrm{mmol})$ and a catalytic amount of tetrabutylammonium iodide at $80^{\circ} \mathrm{C}$ overnight to give $2.19 \mathrm{~g}(72 \%)$ of $25 \mathrm{~d}$ as a colorless foam. A crystalline sample (rosettes of fine transparent needles) for X-ray analysis was obtained by crystallization from a mixture of ethyl acetate and 2-propanol. m.p. $136-137^{\circ} \mathrm{C}$ (DSC); $[\alpha]_{\mathrm{D}}^{20}=+14.5\left(c 0.725\right.$, acetone); ${ }^{1} \mathrm{H} \mathrm{NMR}\left(500 \mathrm{MHz}, \mathrm{CDCl}_{3}\right.$, mixture of rotamers $) \delta 7.40\left(\mathrm{AA}^{\prime} \mathrm{XX}^{\prime}, J_{\mathrm{AX}}=\right.$ 8.7 Hz, 0.8H), $7.38\left(\mathrm{AA}^{\prime} X^{\prime}{ }^{\prime}, J_{\mathrm{AX}}=8.7 \mathrm{~Hz}, 1.2 \mathrm{H}\right), 7.18-7.15(\mathrm{~m}, 1 \mathrm{H}), 7.10(\mathrm{~d}, 1.7 \mathrm{~Hz}, 0.4 \mathrm{H}), 6.92(\mathrm{~d}, 1.7$ $\mathrm{Hz}, 0.6 \mathrm{H}), 6.91-6.86$ (overlapping $\mathrm{AA}^{\prime} X X^{\prime}$ spin systems, $\left.J_{\mathrm{AX}}=8.7 \mathrm{~Hz}, 2 \mathrm{H}\right), 4.72-4.60(\mathrm{~m}, 2 \mathrm{H}), 4.60-4.54$ $(\mathrm{m}, 1 \mathrm{H}), 4.51-4.41(\mathrm{~m}, 1.4 \mathrm{H}), 4.30(\mathrm{q}, 7.1 \mathrm{~Hz}, 2 \mathrm{H}), 4.14(\mathrm{dd}, 13.6,8.5 \mathrm{~Hz}, 0.6 \mathrm{H}), 3.99(\mathrm{~d}, 13.2 \mathrm{~Hz}, 0.6 \mathrm{H})$, $3.82(\mathrm{~s}, 3 \mathrm{H}), 3.69(\mathrm{~d}, 13.0 \mathrm{~Hz}, 0.4 \mathrm{H}), 3.33(\mathrm{dd}, 13.1,4.9 \mathrm{~Hz}, 0.6 \mathrm{H}), 3.21(\mathrm{dd}, 12.8,5.1 \mathrm{~Hz}, 0.4 \mathrm{H}), 1.47(\mathrm{~s}$, $4 \mathrm{H}), 1.41(\mathrm{~s}, 3 \mathrm{H}), 1.38 / 1.36$ (overlapping tripletts, $7.0 \mathrm{~Hz}, 3 \mathrm{H}), 1.30(\mathrm{~s}, 5 \mathrm{H}), 1.28(\mathrm{~s}, 2 \mathrm{H}), 1.25(\mathrm{~s}, 1 \mathrm{H}) ;{ }^{13} \mathrm{C}$ NMR $\left(125 \mathrm{MHz}, \mathrm{CDCl}_{3}\right.$, mixture of rotamers) $\delta 161.59,161.35,158.32,154.37,154.27,127.03,126.42$, 126.32, 125.37, 124.98, 124.87, 124.81, 122.90, 122.73, 115.33, 115.28, 114.21, 111.87, 111.71, 82.45, 81.48, $80.16,80.00,79.31,78.93,64.69,64.11,60.07,55.33,52.29,51.04,47.68,46.27,28.43,28.09,27.01,26.92$, 
25.04, 14.47; HRMS (ESI) $m / z$ calcd for $\mathrm{C}_{27} \mathrm{H}_{36} \mathrm{~N}_{2} \mathrm{O}_{7} \mathrm{Na}^{+}$523.2415, found 523.2411; Anal. calcd for $\mathrm{C}_{27} \mathrm{H}_{36} \mathrm{~N}_{2} \mathrm{O}_{7}$ : $\mathrm{C}, 64.78 ; \mathrm{H}, 7.25 ; \mathrm{N}, 5.60$. Found $\mathrm{C}, 64.78 ; \mathrm{H}, 7.33 ; \mathrm{N}, 5.54$.

1-\{[(3aR,4R,6aS)-5-(tert-Butoxycarbonyl)-2,2-dimethyltetrahydro-4H-[1,3]dioxolo[4,5-c]pyrrol-4-

yl]methyl\}-4-(4-methoxyphenyl)-1H-pyrrole-2-carboxylic acid (26d). Deploying the same protocol as described for the preparation of compound 26a ethyl ester $25 \mathrm{~d}(1.96 \mathrm{~g}, 3.92 \mathrm{mmol})$ dissolved in 2:1 mixture of THF $(20 \mathrm{~mL})$ and methanol $(10 \mathrm{~mL})$ was treated with a $4 \mathrm{M}$ aqueous solution of sodium hydroxide $(7.5 \mathrm{~mL})$ at $50{ }^{\circ} \mathrm{C}$ overnight to yield $1.73 \mathrm{~g}(94 \%)$ of crude $26 \mathrm{~d}$. An analytical sample of $\mathbf{2 6 \mathrm { d }}$ was obtained by recrystallization from methanol as a colorless and crystalline solid. m.p. $197-199{ }^{\circ} \mathrm{C}$ (DSC); $[\alpha]_{\mathrm{D}}^{20}=+17.8$ (c 0.695, acetone); ${ }^{1} \mathrm{H} \mathrm{NMR}\left(500 \mathrm{MHz}, \mathrm{CDCl}_{3}\right.$, mixture of rotamers) $\delta 7.43-7.37$ (overlapping AA'XX' spin systems, $\left.J_{\mathrm{AX}}=8.7 \mathrm{~Hz}, 2 \mathrm{H}\right), 7.32(\mathrm{~d}, 2.0 \mathrm{~Hz}, 1 \mathrm{H}), 7.18(\mathrm{~s}, 0.4 \mathrm{H}), 6.99(\mathrm{~d}, 1.3 \mathrm{~Hz}$, $0.6 \mathrm{H}), 6.90\left(\mathrm{AA}^{\prime} \mathrm{XX}^{\prime}, J_{\mathrm{AX}}=8.6 \mathrm{~Hz}, 2 \mathrm{H}\right), 4.73-4.55(\mathrm{~m}, 3 \mathrm{H}), 4.51-4.45(\mathrm{~m}, 1.4 \mathrm{H}), 4.13(\mathrm{dd}, 13.7,8.8 \mathrm{~Hz}$, $0.6 \mathrm{H}), 4.04(\mathrm{~d}, 13.2 \mathrm{~Hz}, 0.6 \mathrm{H}), 3.82(\mathrm{~s}, 3 \mathrm{H}), 3.72(\mathrm{~d}, 12.8 \mathrm{~Hz}, 0.4 \mathrm{H}), 3.35(\mathrm{dd}, 13.2,4.8 \mathrm{~Hz}, 0.6 \mathrm{H}), 3.22$ $(\mathrm{dd}, 12.8,5.0 \mathrm{~Hz}, 0.4 \mathrm{H}), 1.47(\mathrm{~s}, 4 \mathrm{H}), 1.42(\mathrm{~s}, 3 \mathrm{H}), 1.31 / 1.30$ (overlapping singlets, $7 \mathrm{H}), 1.26(\mathrm{~s}, 1 \mathrm{H}) ;{ }^{13} \mathrm{C}$ NMR $\left(125 \mathrm{MHz}, \mathrm{CDCl}_{3}\right.$, mixture of rotamers) $\delta 165.17,164.96,158.46,154.35,126.84,126.65,126.47$, 126.36, 125.51, 125.34, 121.65, 121.49, 117.34, 114.26, 111.95, 111.84, 82.40, 81.48, 80.30, 80.19, 79.31, 78.93, $64.63,64.03,55.32,52.27,51.00,47.83,46.47,28.39,28.07,26.99 / 26.89,25.04 ;$ HRMS (ESI) $\mathrm{m} / z$ calcd for $\mathrm{C}_{25} \mathrm{H}_{32} \mathrm{~N}_{2} \mathrm{O}_{7} \mathrm{H}^{+} 473.2282$, found 473.2281; Anal. calcd for $\mathrm{C}_{25} \mathrm{H}_{32} \mathrm{~N}_{2} \mathrm{O}_{7}$ : $\mathrm{C}, 63.55 ; \mathrm{H}, 6.83 ; \mathrm{N}, 5.93$. Found $\mathrm{C}, 63.58 ; \mathrm{H}, 6.88 ; \mathrm{N}, 5.89$.

tert-Butyl(3aR,4R,6aS)-4-[(2-\{[(1H-benzo[d][1,2,3]triazol-1-yl)oxy]carbonyl\}-4-(4-methoxyphen-yl)-1Hpyrrol-1-yl)methyl]-2,2-dimethyltetrahydro-5H-[1,3]dioxolo[4,5-c]pyrrole-5-carboxylate (27d). Applying the same procedure as for the preparation of compound $27 \mathrm{a}$ carboxylic acid $\mathbf{2 6 \mathrm { d }}(1.05 \mathrm{~g}, 2.22 \mathrm{mmol})$ dissolved in dry DMF $(30 \mathrm{~mL})$ was reacted with HBTU $(1.01 \mathrm{~g}, 2.66 \mathrm{mmol})$ in the presence of triethylamine $(0.93 \mathrm{~mL}, 6.67 \mathrm{mmol})$ to yield $1.28 \mathrm{~g}(98 \%)$ of $27 \mathrm{~d}$ as a colorless foam; $[\alpha]_{\mathrm{D}}^{20}=+41.6(c$ $\left.0.700, \mathrm{CHCl}_{3}\right) ;{ }^{1} \mathrm{H} \mathrm{NMR}\left(500 \mathrm{MHz}, \mathrm{CDCl}_{3}\right.$, mixture of rotamers) $88.12-8.07(\mathrm{~m}, 1 \mathrm{H}), 7.67(\mathrm{~d}, 1.6 \mathrm{~Hz}$, $1 \mathrm{H}), 7.64-7.51(\mathrm{~m}, 2 \mathrm{H}), 7.49-7.41(\mathrm{~m}, 3.4 \mathrm{H}), 7.24(\mathrm{~m}, 0.6 \mathrm{H}), 6.94\left(\mathrm{AA}^{\prime} \mathrm{XX}^{\prime}, J_{\mathrm{AX}}=8.7 \mathrm{~Hz}, 2 \mathrm{H}\right), 4.67-4.62$ $(\mathrm{m}, 0.6 \mathrm{H}), 4.61-4.51(\mathrm{~m}, 1.2 \mathrm{H}), 4.51-4.39(\mathrm{~m}, 2.6 \mathrm{H}), 4.22(\mathrm{dd}, 14.8,11.5 \mathrm{~Hz}, 0.6 \mathrm{H}), 4.07(\mathrm{~d}, 13.4 \mathrm{~Hz}, 0.6 \mathrm{H})$, $3.85(\mathrm{~s}, 3 \mathrm{H}), 3.75(\mathrm{~d}, 13.7 \mathrm{~Hz}, 0.4 \mathrm{H}), 3.27-3.17(\mathrm{~m}, 1 \mathrm{H}), 1.45(\mathrm{~s}, 3 \mathrm{H}), 1.41(\mathrm{~s}, 1 \mathrm{H}), 1.39(\mathrm{~s}, 2 \mathrm{H}), 1.30(\mathrm{~s}$, $6 \mathrm{H}), 1.26(\mathrm{~s}, 3 \mathrm{H}) ;{ }^{13} \mathrm{C} \mathrm{NMR}\left(125 \mathrm{MHz}, \mathrm{CDCl}_{3}\right.$, mixture of rotamers) $\delta 158.91,157.05,154.43,154.36$, $143.55,129.49,129.23,129.15,128.78,128.68,126.92,126.67,125.78,124.79,120.59,120.43,118.64,118.52$, $116.77,116.53,114.47,112.12,108.77,108.47,82.41,81.50,80.50,80.34,79.36,79.03,65.04,64.03,55.37$, $51.99,50.83,48.22,47.03,28.35,28.09,26.91,26.84,24.94$; HRMS (ESI) $m / z$ calcd for $\mathrm{C}_{31} \mathrm{H}_{35} \mathrm{~N}_{5} \mathrm{O}_{7} \mathrm{Na}^{+}$ 612.2429 , found 612.2429 .

tert-Butyl(3aR,4R,6aS)-4-\{[2-(\{4-[3-(4-fluorophenyl)-5-isopropylisoxazol-4-yl]pyridin-2-yl\}carba-moyl)-4(4-methoxyphenyl)-1H-pyrrol-1-yl]methyl\}-2,2-dimethyltetrahydro-5H-[1,3]dioxolo[4,5-c]pyrrole-5-carboxylate (28d). Following the procedure as described for the preparation of compound 28a isoxazole 30 (266 $\mathrm{mg}, 0.895 \mathrm{mmol})$ dissolved in dry DMF $(5 \mathrm{~mL})$ was deprotonated with sodium hydride $(60 \%$ in mineral oil; $32 \mathrm{mg}, 0.80 \mathrm{mmol})$. Subsequently, a solution of compound $27 \mathrm{~d}$ ( $309 \mathrm{mg}, 0.524 \mathrm{mmol})$ in dry DMF $(5 \mathrm{~mL})$ was added dropwise via cannula and the reaction was stirred at room temperature for $48 \mathrm{~h}$ to obtain $220 \mathrm{mg}(56 \%)$ of $\mathbf{2 8 d}$ as a colorless foam. $[\alpha]_{\mathrm{D}}^{20}=+36.8$ (c 0.595, acetone); ${ }^{1} \mathrm{H}$ NMR $\left(500 \mathrm{MHz}, \mathrm{CDCl}_{3}\right.$, mixture of rotamers) $\delta 8.49(\mathrm{~s}, 0.4 \mathrm{H}), 8.45(\mathrm{~s}, 0.6 \mathrm{H}), 8.27-8.22(\mathrm{~m}, 2 \mathrm{H}), 7.45\left(\mathrm{AA}^{\prime} \mathrm{BB}^{\prime} \mathrm{X}\right.$, $\left.J_{\mathrm{AB}}=8.5, J_{\mathrm{AF}}=5.4 \mathrm{~Hz}, 2 \mathrm{H}\right), 7.42-7.36(\mathrm{~m}, 2 \mathrm{H}), 7.14(\mathrm{~s}, 0.4 \mathrm{H}), 7.08-7.02(\mathrm{~m}, 3 \mathrm{H}), 6.99(\mathrm{~s}, 0.6 \mathrm{H}), 6.92$ $\left(\mathrm{AA}^{\prime} \mathrm{XX}^{\prime}, J_{\mathrm{AX}}=8.7 \mathrm{~Hz}, 2 \mathrm{H}\right), 6.78-6.73(\mathrm{~m}, 1 \mathrm{H}), 4.77-4.66(\mathrm{~m}, 1.4 \mathrm{H}), 4.64-4.56(\mathrm{~m}, 1.6 \mathrm{H}), 4.52-4.44(\mathrm{~m}$, 1H), $4.39(\mathrm{dd}, 13.6,5.6 \mathrm{~Hz}, 0.4 \mathrm{H}), 4.29(\mathrm{dd}, 13.6,8.4 \mathrm{~Hz}, 0.6 \mathrm{H}), 3.98(\mathrm{~d}, 13.1 \mathrm{~Hz}, 0.6 \mathrm{H}), 3.83(\mathrm{~s}, 3 \mathrm{H}), 3.73$ $(\mathrm{d}, 12.8 \mathrm{~Hz}, 0.4 \mathrm{H}), 3.32(\mathrm{dd}, 13.1,4.8 \mathrm{~Hz}, 0.6 \mathrm{H}), 3.30-3.22$ (overlapping signals: $\mathrm{m}, 0.4 \mathrm{H} ; 3.27, \mathrm{sp}, 6.9$ $\mathrm{Hz}, 1 \mathrm{H}), 1.44(\mathrm{~s}, 4 \mathrm{H}), 1.43-1.39$ (overlapping signals: 1.41, s, 3H; 1.41, d, $6.9 \mathrm{~Hz}, 6 \mathrm{H}), 1.31(\mathrm{~s}, 5 \mathrm{H}), 1.27$ (s, 2H), $1.23(\mathrm{~s}, 1 \mathrm{H}) ;{ }^{19} \mathrm{~F}$ NMR $\left(470 \mathrm{MHz}, \mathrm{CDCl}_{3}\right) \delta-110.91,-110.96 ;{ }^{13} \mathrm{C} \mathrm{NMR}\left(125 \mathrm{MHz}, \mathrm{CDCl}_{3}\right.$, mixture of rotamers) $\delta 175.54,164.61 / 162.62(\mathrm{~d}, 248.4 \mathrm{~Hz}), 160.07,159.66,159.41,158.53,154.39,154.25$, $152.29,152.22,148.06,141.25,130.56 / 130.50$ (d, 7.8 Hz), 126.66, 126.62, 126.41, 126.36, 125.43, 125.31, 125.25, 125.11, 125.04, 124.77, 124.74, 120.46, 115.91/115.74 (d, $21.6 \mathrm{~Hz}), 114.49,114.46,114.37,114.35$, $112.15,111.85,111.73,111.03,82.41,81.50,80.18,80.00,79.48,79.00,64.87,64.04,55.36,52.17,51.06$, 
$47.56,46.41,28.39,28.15,26.98,26.92,26.67,25.05,21.01$; HRMS (ESI) $m / z$ calcd for $\mathrm{C}_{42} \mathrm{H}_{46} \mathrm{FN}_{5} \mathrm{O}_{7} \mathrm{H}^{+}$ 752.3454, found 752.3461. Anal. calcd for $\mathrm{C}_{42} \mathrm{H}_{46} \mathrm{FN}_{5} \mathrm{O}_{7} \cdot 0.1\left(\mathrm{CHCl}_{3}\right): \mathrm{C}, 66.20 ; \mathrm{H}, 6.08 ; \mathrm{N}, 9.17$. Found C, 66.18; $\mathrm{H}, 6.09 ; \mathrm{N}, 9.13$.

$(2 R, 3 R, 4 S)-2-\{[2-(\{4-[3-(4-F l u o r o p h e n y l)-5-i s o p r o p y l i s o x a z o l-4-y l] p y r i d i n-2-y l\}$ carbamoyl)-3-(4methoxyphenyl)-1H-pyrrol-1-yl]methyl\}-3,4-dihydroxypyrrolidin-1-ium trifluoroacetate (29d). Employing the same method as for the preparation of $29 \mathrm{c}$ compound $28 \mathrm{~d}(161 \mathrm{mg}, 0.214 \mathrm{mmol})$ was treated with a mixture of trifluoroacetic acid $(4.75 \mathrm{~mL})$ and water $(0.5 \mathrm{~mL})$ at $0{ }^{\circ} \mathrm{C}$ for $2.5 \mathrm{~h}$ to yield $119 \mathrm{mg}$ (77\%) of $\mathbf{2 9 d}$ as a yellow semisolid. Pure $29 \mathrm{~d}$ was obtained by subsequent recrystallization from ethyl acetate and petroleum ether. m.p. $191-193{ }^{\circ} \mathrm{C}(\mathrm{DSC}) ;[\alpha]_{\mathrm{D}}^{20}=-2.4(c 0.655, \mathrm{MeOH}) ;{ }^{1} \mathrm{H}$ NMR $(500$ $\left.\mathrm{MHz}, \mathrm{CD}_{3} \mathrm{OD}\right) \delta 8.38(\mathrm{~d}, 5.3 \mathrm{~Hz}, 1 \mathrm{H}), 8.09-8.07(\mathrm{~m}, 1 \mathrm{H}), 7.52\left(\mathrm{AA}^{\prime} \mathrm{XX}^{\prime}, J_{\mathrm{AX}}=8.8 \mathrm{~Hz}, 2 \mathrm{H}\right), 7.49-7.44$ (overlapping signals: 7.48, d, $1.7 \mathrm{~Hz}, 1 \mathrm{H}$ and 7.46, $\left.\mathrm{AA}^{\prime} \mathrm{BB}^{\prime} \mathrm{X}, J_{\mathrm{AB}}=8.9, J_{\mathrm{AF}}=5.3 \mathrm{~Hz}, 2 \mathrm{H}\right), 7.42(\mathrm{~d}, 1.9$ $\mathrm{Hz}, 1 \mathrm{H}), 7.14\left(\mathrm{AA}^{\prime} \mathrm{BB}^{\prime} X, J_{\mathrm{AB}}=J_{\mathrm{BF}}=8.9 \mathrm{~Hz}, 2 \mathrm{H}\right), 6.99(\mathrm{dd}, 5.1,1.5 \mathrm{~Hz}, 1 \mathrm{H}), 6.94\left(\mathrm{AA}^{\prime} X X^{\prime}, J_{\mathrm{AX}}=8.8 \mathrm{~Hz}\right.$, 2H), $4.73(\mathrm{dd}, 15.2,8.8 \mathrm{~Hz}, 1 \mathrm{H}), 4.68(\mathrm{dd}, 15.2,3.8 \mathrm{~Hz}, 1 \mathrm{H}), 4.25(\mathrm{dt}, 3.8,1.6 \mathrm{~Hz}, 1 \mathrm{H}), 4.12(\mathrm{dd}, 8.8,3.9$ $\mathrm{Hz}, 1 \mathrm{H}), 3.91(\mathrm{td}, 8.8,3.8 \mathrm{~Hz}, 1 \mathrm{H}), 3.81(\mathrm{~s}, 3 \mathrm{H}), 3.48(\mathrm{dd}, 12.5,3.9 \mathrm{~Hz}, 1 \mathrm{H}), 3.28(\mathrm{sp}, 7.0 \mathrm{~Hz}, 1 \mathrm{H}), 3.24$ $(\mathrm{dd}, 12.5,1.7 \mathrm{~Hz}, 1 \mathrm{H}), 1.38(\mathrm{~d}, 7.0 \mathrm{~Hz}, 6 \mathrm{H}) ;{ }^{19} \mathrm{~F}$ NMR $\left(470 \mathrm{MHz}, \mathrm{CD}_{3} \mathrm{OD}\right) \delta-76.90,-112.83 ;{ }^{13} \mathrm{C}$ NMR $\left(125 \mathrm{MHz}, \mathrm{CD}_{3} \mathrm{OD}\right) \delta 176.93,166.15 / 164.18(\mathrm{~d}, 247.3 \mathrm{~Hz}), 162.99,161.47,160.20,153.67,149.90,142.32$, $131.83 / 131.76(\mathrm{~d}, 8.5 \mathrm{~Hz}), 127.90,127.40,127.11,126.21 / 126.18$ (d, $3.0 \mathrm{~Hz}), 122.40,117.20,116.98 / 116.80$ (d, $22.1 \mathrm{~Hz}), 115.37,114.13,113.61,74.82,70.83,63.72,55.81,51.13,48.70,27.98,21.25 ;$ HRMS (ESI) $\mathrm{m} / \mathrm{z}$ calcd for $\mathrm{C}_{34} \mathrm{H}_{34} \mathrm{FN}_{5} \mathrm{O}_{5} \mathrm{H}^{+} 612.2617$, found 612.2616. Anal. calcd for $\mathrm{C}_{34} \mathrm{H}_{34} \mathrm{FN}_{5} \mathrm{O}_{5}{ }^{+} \cdot \mathrm{CF}_{3} \mathrm{COOH}$ : C, $59.58 ; \mathrm{H}, 4.86 ; \mathrm{N}, 9.65$. Found C, 59.49; H, 4.88; N, 9.50.

tert-Butyl(3aS,4S,6aR)-4-\{[2-(ethoxycarbonyl)-4-(4-methoxyphenyl)-1H-pyrrol-1-yl]methyl\}-2,2dimethyltetrahydro-5H-[1,3]dioxolo[4,5-c]pyrrole-5-carboxylate (25e). Using the same procedure as for the preparation of compound $25 \mathrm{c}$ crude mesylate $(+)-20(1.22 \mathrm{~g}, 3.47 \mathrm{mmol})$ dissolved in dry DMF $(20 \mathrm{~mL})$ was reacted with pyrrole $24 \mathrm{a}(712 \mathrm{mg}, 2.90 \mathrm{mmol})$, cesium carbonate $(2.08 \mathrm{~g}, 6.38 \mathrm{mmol})$ and a catalytic amount of tetra- $n$-butylammonium iodide to afford $1.12 \mathrm{~g}(77 \%)$ of $25 \mathbf{e}$ as a colorless foam. $[\alpha]_{D}^{20}=-15.4$ (c 0.695 , acetone). All spectroscopic data was consistent with that reported for its enantiomer 25d. HRMS (ESI) $m / z$ calcd for $\mathrm{C}_{27} \mathrm{H}_{36} \mathrm{~N}_{2} \mathrm{O}_{7} \mathrm{Na}^{+}$523.2415, found 523.2411; Anal. calcd for $\mathrm{C}_{27} \mathrm{H}_{36} \mathrm{~N}_{2} \mathrm{O}_{7}$ : $\mathrm{C}, 64.78 ; \mathrm{H}, 7.25 ; \mathrm{N}, 5.60$. Found $\mathrm{C}, 64.61 ; \mathrm{H}, 7.37 ; \mathrm{N}, 5.59$.

1-\{[(3aS,4S,6aR)-5-(tert-Butoxycarbonyl)-2,2-dimethyltetrahydro-4H-[1,3]dioxolo[4,5-c]pyrrol-4yl]methyl)-4-(4-methoxyphenyl)-1H-pyrrole-2-carboxylic acid (26e). Applying the same protocol as for the preparation of compound 26a ethyl ester 25e $(1.01 \mathrm{~g}, 2.02 \mathrm{mmol})$ was dissolved in a 2:1 mixture of THF and methanol $(12 \mathrm{~mL} / 6 \mathrm{~mL})$ and treated with an aqueous solution of potassium hydroxide $(8 \mathrm{M}$, $3.75 \mathrm{~mL}$ ) at $50{ }^{\circ} \mathrm{C}$ overnight to afford $983 \mathrm{mg}$ (quant.) of crude 26e as a light-yellow foam which was subsequently deployed in the next reaction step without further purification. An analytical sample was obtained by recrystallization from methanol. m.p. $196-199{ }^{\circ} \mathrm{C}$ (DSC). $[\alpha]_{\mathrm{D}}^{20}=-17.7$ (c 0.515, acetone). All spectroscopic data was consistent with the data reported for its enantiomer 26d. HRMS (ESI) $m / z$ calcd for $\mathrm{C}_{25} \mathrm{H}_{32} \mathrm{~N}_{2} \mathrm{O}_{7} \mathrm{Na}^{+} 495.2102$, found 495.2102; Anal. calcd for $\mathrm{C}_{25} \mathrm{H}_{32} \mathrm{~N}_{2} \mathrm{O}_{7}$ : $\mathrm{C}, 63.55$; $\mathrm{H}, 6.83 ; \mathrm{N}, 5.93$. Found $\mathrm{C}, 63.47 ; \mathrm{H}, 6.89 ; \mathrm{N}, 5.91$.

tert-Butyl(3aS,4S,6aR)-4-\{[2-\{[(1H-benzo[d][1,2,3]triazol-1-yl)oxy]carbonyl\}-4-(4-methoxyphenyl)-1Hpyrrol-1-yl]methyl\}-2,2-dimethyltetrahydro-5H-[1,3]dioxolo[4,5-c]pyrrole-5-carboxylate (27e). Using the same method as described for the preparation of compound 27a crude carboxylic acid 26e $(916 \mathrm{mg}$, $1.94 \mathrm{mmol})$ dissolved in dry DMF $(20 \mathrm{~mL})$ was reacted with HBTU $(894 \mathrm{mg}, 2.36 \mathrm{mmol})$ in the presence of triethylamine $(0.83 \mathrm{~mL}, 5.96 \mathrm{mmol})$ to yield $1.05 \mathrm{~g}(92 \%)$ of $27 \mathbf{e}$ as a colorless foam. $[\alpha]_{\mathrm{D}}^{20}=-39.7(\mathrm{c}$ $\left.0.635, \mathrm{CHCl}_{3}\right)$; All spectroscopic data was consistent with the data described for its enantiomer $27 \mathrm{~d}$. HRMS (ESI) $m / z$ calcd for $\mathrm{C}_{31} \mathrm{H}_{35} \mathrm{~N}_{5} \mathrm{O}_{7} \mathrm{Na}^{+}$612.2429, found 612.2437 .

tert-Butyl(3aS,4S,6aR)-4-\{[2-(\{4-[3-(4-fluorophenyl)-5-isopropylisoxazol-4-yl]pyridin-2-yl\}carbamoyl)-4(4-methoxyphenyl)-1H-pyrrol-1-yl]methyll-2,2-dimethyltetrahydro-5H-[1,3]di-oxolo[4,5-c]pyrrole-5-carboxylate (28e). Following the procedure outlined for the preparation of compound 28a isoxazole 30 (474 mg, $1.59 \mathrm{mmol})$ dissolved in dry DMF $(14 \mathrm{~mL})$ was deprotonated with sodium hydride (60\% in mineral oil; $75 \mathrm{mg}, 1.88 \mathrm{mmol}$ ) and compound $27 \mathrm{e}(500 \mathrm{mg}, 0.848 \mathrm{mmol}$ ) was subsequently introduced in one 
portion at room temperature to yield $335 \mathrm{mg}(53 \%)$ of $28 \mathbf{e}$ as a light-yellow resin. $[\alpha]_{\mathrm{D}}^{20}=-32.5(c$ 0.76, acetone). All spectroscopic data of 28e was consistent with that reported for its enantiomer $\mathbf{2 8 d}$. HRMS (ESI) $m / z$ calcd for $\mathrm{C}_{42} \mathrm{H}_{46} \mathrm{FN}_{5} \mathrm{O}_{7} \mathrm{Na}^{+} 774.3273$, found 774.3269. Anal. calcd for $\mathrm{C}_{42} \mathrm{H}_{46} \mathrm{FN}_{5} \mathrm{O}_{7}$ : C, 67.10; H, 6.17; N, 9.31. Found C, 67.45; H, 6.53; N, 8.92.

$(2 S, 3 S, 4 R)-2-\{[2-(\{4-[3-(4-F l u o r o p h e n y l)-5-i s o p r o p y l i s o x a z o l-4-y l] p y r i d i n-2-y l\}$ carbamoyl)-3-(4methoxyphenyl)-1H-pyrrol-1-yl]methyl\}-3,4-dihydroxypyrrolidin-1-ium trifluoroacetate (29e). Employing the same procedure as outlined for the preparation of $29 \mathrm{c}$ compound $28 \mathrm{e}(234 \mathrm{mg}, 0.311 \mathrm{mmol})$ was treated with a mixture of TFA $(6.8 \mathrm{~mL})$ and water $(0.76 \mathrm{~mL})$ at $0{ }^{\circ} \mathrm{C}$ for $3 \mathrm{~h}$ and the crude product was purified by automated flash column chromatography (silica gel, methanol/dichloromethane $0-10 \%)$ to give $179 \mathrm{mg}$ (79\%) of $29 \mathrm{e}$ as a light-yellow resin. Subsequent recrystallization from ethyl acetate/petroleum ether provided $145 \mathrm{mg}$ of pure 29e as a colorless solid. m.p. $188-191{ }^{\circ} \mathrm{C}$ (DSC); $[\alpha]_{\mathrm{D}}^{20}=+2.6(c 0.53, \mathrm{MeOH})$. All spectroscopic data was consistent with the data reported for its enantiomer 29d. HRMS (ESI) $m / z$ calcd for $\mathrm{C}_{34} \mathrm{H}_{34} \mathrm{FN}_{5} \mathrm{O}_{5} \mathrm{H}^{+}$612.2617, found 612.2620. Anal. calcd for $\mathrm{C}_{34} \mathrm{H}_{34} \mathrm{FN}_{5} \mathrm{O}_{5}{ }^{+} \cdot \mathrm{CF}_{3} \mathrm{COOH}: \mathrm{C}, 59.58 ; \mathrm{H}, 4.86 ; \mathrm{N}, 9.65$. Found $\mathrm{C}, 59.71 ; \mathrm{H}, 4.98 ; \mathrm{N}, 9.68$.

tert-Butyl(3aR,4R,6aS)-4-\{[4-(2,4-dimethoxyphenyl)-2-(ethoxycarbonyl)-1H-pyrrol-1-yl]methyl\}-2,2dimethyltetrahydro-5H-[1,3]dioxolo[4,5-c]pyrrole-5-carboxylate (25f). Employing the same procedure as for the preparation of compound 25c crude mesylate (-)-20 (2.58 g, $7.34 \mathrm{mmol})$ dissolved in dry DMF (50 $\mathrm{mL})$ was reacted with pyrrole $24 \mathrm{~b}(1.68 \mathrm{~g}, 6.10 \mathrm{mmol})$ and cesium carbonate $(4.48 \mathrm{~g}, 13.8 \mathrm{mmol})$ to afford $1.86 \mathrm{~g}(57 \%)$ of $25 \mathrm{f}$ as a colorless oil that solidified on standing. $[\alpha]_{\mathrm{D}}^{20}=+13.2(c 1.105$, acetone); ${ }^{1} \mathrm{H}$ NMR $\left(500 \mathrm{MHz}, \mathrm{CDCl}_{3}\right.$, mixture of rotamers) $\delta 7.42-7.37(\mathrm{~m}, 1 \mathrm{H}), 7.32(\mathrm{~d}, 1.6 \mathrm{~Hz}, 0.4 \mathrm{H}), 7.25(\mathrm{~d}, 1.7$ $\mathrm{Hz}, 0.4 \mathrm{H}), 7.24(\mathrm{~d}, 1.7 \mathrm{~Hz}, 0.6 \mathrm{H}), 7.21(\mathrm{~d}, 1.7 \mathrm{~Hz}, 0.6 \mathrm{H}), 6.53-6.49(\mathrm{~m}, 2 \mathrm{H}), 4.67(\mathrm{dd}, 13.9,7.8 \mathrm{~Hz}, 0.4 \mathrm{H})$, 4.64-4.57 (m, 2.2H), 4.55 (dd, 14.0, 4.7 Hz, 0.4H), 4.51 (t, $5.2 \mathrm{~Hz}, 0.4 \mathrm{H}), 4.47-4.41(\mathrm{~m}, 1 \mathrm{H}), 4.32-4.24$ (overlapping signals: 4.29, q, 7.0 Hz, $2 \mathrm{H}$ and $\mathrm{m}, 0.6 \mathrm{H}), 3.91(\mathrm{~d}, 13.0 \mathrm{~Hz}, 0.6 \mathrm{H}), 3.86(\mathrm{~s}, 1 \mathrm{H}), 3.85(\mathrm{~s}, 2 \mathrm{H})$, $3.82(\mathrm{~s}, 3 \mathrm{H}), 3.66(\mathrm{~d}, 13.2 \mathrm{~Hz}, 0.4 \mathrm{H}), 3.33(\mathrm{dd}, 13.1,4.2 \mathrm{~Hz}, 0.6 \mathrm{H}), 3.25(\mathrm{dd}, 12.7,5.3 \mathrm{~Hz}, 0.4 \mathrm{H}), 1.47$ (s, $3 \mathrm{H}), 1.41(\mathrm{~s}, 3 \mathrm{H}), 1.39-1.33$ (overlapping signals: $\mathrm{m}, 3 \mathrm{H}$ and $1.34, \mathrm{~s}, 6 \mathrm{H}), 1.27(\mathrm{~s}, 2 \mathrm{H}), 1.24(\mathrm{~s}, 1 \mathrm{H}) ;{ }^{13} \mathrm{C}$ NMR $\left(125 \mathrm{MHz}, \mathrm{CDCl}_{3}\right.$, mixture of rotamers) $\delta 161.70,161.52,159.31,157.18,154.39,154.14,128.62$, $128.35,128.26,121.67,120.85,120.60,117.08,116.84,116.13,111.75,111.58,104.73,98.93,82.42,81.40$, $80.03,79.91,79.30,78.78,64.61,63.92,59.94,55.41,55.37,55.29,52.39,51.27,47.47,46.21,28.44,28.12$, 27.06, 26.98, 25.08, 14.50; HRMS (ESI) $m / z$ calcd for $\mathrm{C}_{28} \mathrm{H}_{38} \mathrm{~N}_{2} \mathrm{O}_{8} \mathrm{Na}^{+}$553.2520, found 553.2529.

1-\{[(3aR,4R,6aS)-5-(tert-Butoxycarbonyl)-2,2-dimethyltetrahydro-4H-[1,3]dioxolo[4,5-c]pyrrol-4yl]methyl\}-4-(2,4-dimethoxyphenyl)-1H-pyrrole-2-carboxylic acid (26f). Applying the same procedure as reported for the preparation of carboxylic acid 25a ethyl ester $25 \mathrm{f}(1.21 \mathrm{~g}, 2.28 \mathrm{mmol})$ dissolved in a 2:1 mixture of THF $(12.5 \mathrm{~mL})$ and methanol $(6.5 \mathrm{~mL})$ was treated with a $4 \mathrm{M}$ aqueous sodium hydroxide solution $(4.4 \mathrm{~mL})$ at $50{ }^{\circ} \mathrm{C}$ overnight to obtain $1.05 \mathrm{~g}(92 \%)$ of $\mathbf{2 6 f}$ as a light-yellow solid. An analytical sample of $\mathbf{2 6 f}$ was obtained as colorless crystals by re-crystallization from ethyl acetate/petroleum ether. m.p. $180-181^{\circ} \mathrm{C}$ (DSC); $[\alpha]_{\mathrm{D}}^{20}=+12.6$ (c 0.840 , acetone); ${ }^{1} \mathrm{H} \mathrm{NMR}\left(500 \mathrm{MHz}, \mathrm{CDCl}_{3}\right.$, mixture of rotamers) $\delta 7.43-7.37(\mathrm{~m}, 2 \mathrm{H}), 7.27-7.25(\mathrm{~m}, 1 \mathrm{H}), 6.54-6.50(\mathrm{~m}, 2 \mathrm{H}), 4.71-4.51(\mathrm{~m}, 3.4 \mathrm{H}), 4.51-4.44(\mathrm{~m}$, 1H), $4.25(\mathrm{dd}, 13.7,7.8 \mathrm{~Hz}, 0.6 \mathrm{H}), 3.96(\mathrm{~d}, 13.0 \mathrm{~Hz}, 0.6 \mathrm{H}), 3.87(\mathrm{~s}, 1 \mathrm{H}), 3.86(\mathrm{~s}, 2 \mathrm{H}), 3.83(\mathrm{~s}, 3 \mathrm{H}), 3.70(\mathrm{~d}$, $13.1 \mathrm{~Hz}, 0.4 \mathrm{H}), 3.34(\mathrm{dd}, 13.0,4.9 \mathrm{~Hz}, 0.6 \mathrm{H}), 3.25$ (dd, 12.7, $5.2 \mathrm{~Hz}, 0.4 \mathrm{H}), 1.47(\mathrm{~s}, 3 \mathrm{H}), 1.42(\mathrm{~s}, 3 \mathrm{H}), 1.35$ $(\mathrm{s}, 6 \mathrm{H}), 1.29(\mathrm{~s}, 2 \mathrm{H}), 1.26(\mathrm{~s}, 1 \mathrm{H}) ;{ }^{13} \mathrm{C} \mathrm{NMR}\left(125 \mathrm{MHz}, \mathrm{CDCl}_{3}\right.$, mixture of rotamers) $\delta 165.50,165.32$, $159.49,157.21,154.40,154.20,130.07,129.81,128.38,128.32,121.48,121.22,120.43,119.26,119.10,115.78$, $111.87,111.71,104.78,98.97,82.40,81.41,80.18,80.09,79.33,78.82,64.55,63.89,55.43,55.38,55.31,52.40$, $51.25,47.67,46.40,28.43,28.13,27.05,26.98,25.11$; HRMS (ESI) $m / z$ calcd for $\mathrm{C}_{26} \mathrm{H}_{34} \mathrm{~N}_{2} \mathrm{O}_{8} \mathrm{Na}^{+} 525.2207$, found 525.2220. Anal. calcd for $\mathrm{C}_{26} \mathrm{H}_{34} \mathrm{~N}_{2} \mathrm{O}_{8}$ : C, 62.14; $\mathrm{H}, 6.82 ; \mathrm{N}, 5.57$. Found C, 62.13; H, 6.96; N, 5.48. tert-Butyl(3aR,4R,6aS)-4-\{[2-\{[(1H-benzo[d][1,2,3]triazol-1-yl)oxy]carbonyl\}-4-(2,4-dimethoxyphenyl)1H-pyrrol-1-yl]methyl\}-2,2-dimethyltetrahydro-5H-[1,3]dioxolo[4,5-c]pyrrole-5-carboxylate (27f). Deploying the same procedure as for the preparation of compound $27 \mathrm{a}$ carboxylic acid $\mathbf{2 6 f}(317 \mathrm{mg}, 0.631 \mathrm{mmol})$ dissolved in dry DMF (6 mL) was reacted with HBTU $(304 \mathrm{mg}, 0.802 \mathrm{mmol})$ in the presence of triethylamine $(0.3 \mathrm{~mL}, 2.15 \mathrm{mmol})$ to give $335 \mathrm{mg}(86 \%)$ of $27 \mathrm{f}$ as a colorless resin. $[\alpha]_{\mathrm{D}}^{20}=+34.6(c$ $\left.0.735, \mathrm{CHCl}_{3}\right) ;{ }^{1} \mathrm{H} \mathrm{NMR}\left(500 \mathrm{MHz}, \mathrm{CDCl}_{3}\right.$, mixture of rotamers) $\delta 8.11-8.06(\mathrm{~m}, 1 \mathrm{H}), 7.78-7.75(\mathrm{~m}, 1 \mathrm{H})$, 
7.65-7.49 (m, 3H), 7.47-7.41 (m, 2H), 6.58-6.54 (m, 2H), $4.61(\mathrm{t}, 5.0 \mathrm{~Hz}, 0.6 \mathrm{H}), 4.57(\mathrm{dd}, 12.3,6.1 \mathrm{~Hz}$, $0.4 \mathrm{H}), 4.54-4.39(\mathrm{~m}, 3.4 \mathrm{H}), 4.27(\mathrm{dd}, 15.41,10.4 \mathrm{~Hz}, 0.6 \mathrm{H}), 4.01(\mathrm{~d}, 13.3 \mathrm{~Hz}, 0.6 \mathrm{H}), 3.91(\mathrm{~s}, 1 \mathrm{H}), 3.90(\mathrm{~s}$, $2 \mathrm{H}), 3.85(\mathrm{~s}, 3 \mathrm{H}), 3.72(\mathrm{~d}, 12.8 \mathrm{~Hz}, 0.4 \mathrm{H}), 3.27-3.19$ (overlapping signals: $\mathrm{m}, 0.4 \mathrm{H}$ and $3.24, \mathrm{dd}, 13.2$, $4.7 \mathrm{~Hz}, 0.6 \mathrm{H}), 1.45(\mathrm{~s}, 3 \mathrm{H}), 1.41(\mathrm{~s}, 1 \mathrm{H}), 1.40(\mathrm{~s}, 2 \mathrm{H}), 1.31(\mathrm{~s}, 6 \mathrm{H}), 1.26(\mathrm{~s}, 3 \mathrm{H}) ;{ }^{13} \mathrm{C} \mathrm{NMR}(125 \mathrm{MHz}$, $\mathrm{CDCl}_{3}$, mixture of rotamers) $\delta 159.91,157.27,157.10,154.35,154.22,143.49,132.53,132.07,129.23$, $129.16,128.67,128.56,128.52,128.40,124.70,122.87,122.60,120.54,120.49,120.33,120.26,115.47,115.40$, 114.90, 114.86, 111.99, 111.92, 108.79, 108.48, 104.91, 98.99, 82.36, 81.36, 80.32, 80.19, 79.28, 78.90, 64.91, 63.78, 55.41, 55.33, 52.05, 50.92, 47.98, 46.84, 28.31, 28.01, 26.91, 26.83, 24.93; HRMS (ESI) $\mathrm{m} / \mathrm{z}$ calcd for $\mathrm{C}_{32} \mathrm{H}_{37} \mathrm{~N}_{5} \mathrm{O}_{8} \mathrm{Na}^{+} 642.2534$, found 642.2530 .

tert-Butyl(3aR,4R,6aS)-4-\{[4-(2,4-dimethoxyphenyl)-2-(\{4-[3-(4-fluorophenyl)-5-isopropylisoxa-zol-4yl]pyridin-2-yl\}carbamoyl)-1H-pyrrol-1-yl]methyl\}-2,2-dimethyltetrahydro-5H-[1,3]dioxolo

[4,5-c]pyrrole-5-carboxylate (28f). Following the same method as described for the preparation of compound 28a isoxazole 30 (196 mg, $0.659 \mathrm{mmol}$ ) dissolved in dry DMF (5 mL) was initially deprotonated with sodium hydride ( $60 \%$ in mineral oil; $24.7 \mathrm{mg}, 0.618 \mathrm{mmol})$ which was then followed by introducing dropwise a solution of compound $27 \mathrm{f}(230 \mathrm{mg}, 0.371 \mathrm{mmol})$ in dry DMF $(5 \mathrm{~mL})$ via cannula to give $103 \mathrm{mg}(36 \%)$ of $\mathbf{2 8 f}$ as a colorless foam. $[\alpha]_{\mathrm{D}}^{20}=+26.2\left(c 0.775\right.$, acetone); ${ }^{1} \mathrm{H}$ NMR (500 $\mathrm{MHz}, \mathrm{CDCl}_{3}$, mixture of rotamers) $\delta 8.49\left(\mathrm{~s}_{\mathrm{br}}, 0.4 \mathrm{H}\right), 8.46\left(\mathrm{~s}_{\mathrm{br}}, 0.6 \mathrm{H}\right), 8.28-8.23(\mathrm{~m}, 2 \mathrm{H}), 7.45\left(\mathrm{AA}^{\prime} \mathrm{BB}^{\prime} \mathrm{X}\right.$, $\left.J_{\mathrm{AB}}=8.4, J_{\mathrm{AF}}=5.5 \mathrm{~Hz}, 2 \mathrm{H}\right), 7.39-7.35(\mathrm{~m}, 1 \mathrm{H}), 7.34(\mathrm{~s}, 0.4 \mathrm{H}), 7.23(\mathrm{~s}, 0.6 \mathrm{H}), 7.18-7.14(\mathrm{~m}, 1 \mathrm{H}), 7.05$ $\left(\mathrm{AA}^{\prime} \mathrm{BB}^{\prime} \mathrm{X}, J_{\mathrm{AB}}=J_{\mathrm{BF}}=8.6 \mathrm{~Hz}, 2 \mathrm{H}\right), 6.74(\mathrm{t}, 4.5 \mathrm{~Hz}, 1 \mathrm{H}), 6.56-6.52(\mathrm{~m}, 2 \mathrm{H}), 4.74-4.55(\mathrm{~m}, 3 \mathrm{H}), 4.53-4.43$ $(\mathrm{m}, 1.4 \mathrm{H}), 4.37(\mathrm{dd}, 13.4,7.6 \mathrm{~Hz}, 0.6 \mathrm{H}), 3.92(\mathrm{~d}, 13.1 \mathrm{~Hz}, 0.6 \mathrm{H}), 3.89(\mathrm{~s}, 1 \mathrm{H}), 3.88(\mathrm{~s}, 2 \mathrm{H}), 3.84(\mathrm{~s}, 3 \mathrm{H}), 3.71$ (d, $12.9 \mathrm{~Hz}, 0.4 \mathrm{H}$ ), 3.36-3.22 (overlapping signals: 3.33 , dd, $13.1,4.9 \mathrm{~Hz}, 0.6 \mathrm{H} ; \mathrm{m}, 0.4 \mathrm{H}$ and 3.27, sp, $7.0 \mathrm{~Hz}, 1 \mathrm{H}), 1.45(\mathrm{~s}, 3.5 \mathrm{H}), 1.43-1.39$ (overlapping signals, 9H), $1.34(\mathrm{~s}, 5.5 \mathrm{H}), 1.27(\mathrm{~s}, 2 \mathrm{H}), 1.22(\mathrm{~s}, 1 \mathrm{H})$; ${ }^{19} \mathrm{~F}$ NMR $\left(470 \mathrm{MHz}, \mathrm{CDCl}_{3}\right.$, mixture of rotamers) $\delta-110.94,-111.00 ;{ }^{13} \mathrm{C} \mathrm{NMR}\left(125 \mathrm{MHz}, \mathrm{CDCl}_{3}\right.$, mixture of rotamers) $\delta 175.50,164.60 / 162.62(\mathrm{~d}, 247.8 \mathrm{~Hz}), 160.08,159.81,159.56,157.25,154.42,154.17$, 152.43, 152.38, 148.17, 141.11, 130.56/130.49 (d, 8.2 Hz), 128.40, 128.25, 128.20, 124.79, 124.78, 124.17, $124.13,121.08,120.91,120.39,115.90 / 115.73(\mathrm{~d}, 21.7 \mathrm{~Hz}), 114.47,112.98,112.80,112.20,111.76,111.60$, $104.83,99.05,82.40,81.44,80.09,79.92,79.49,78.87,64.78,63.90,55.45,55.34,52.27,51.26,47.42,46.26$, 28.41, 28.15, 27.02, 26.98, 26.66, 25.09, 21.01; HRMS (ESI) $m / z$ calcd for $\mathrm{C}_{43} \mathrm{H}_{48} \mathrm{FN}_{5} \mathrm{O}_{8} \mathrm{Na}^{+}$804.3379, found 804.3383 .

(2R,3R,4S)-2-\{[3-(2,4-Dimethoxyphenyl)-2-(\{4-[3-(4-fluorophenyl)-5-isopropylisoxazol-4-yl]pyridin-2-yl\} carbamoyl)-1H-pyrrol-1-yl]methyll-3,4-dihydroxypyrrolidin-1-ium trifluoroacetate (29f). Compound $28 \mathrm{f}$ (110 $\mathrm{mg}, 0.141 \mathrm{mmol})$ was treated with a 9:1 mixture of trifluoroacetate $(3.6 \mathrm{~mL})$ and water $(0.4 \mathrm{~mL})$ at $0{ }^{\circ} \mathrm{C}$ for $2.5 \mathrm{~h}$ applying the same protocol as for the preparation of compound $29 \mathrm{c}$ to yield $68.4 \mathrm{mg}(64 \%)$ of 29f as a light-yellow foam. $[\alpha]_{\mathrm{D}}^{20}=-1.14\left(c\right.$ 0.875, MeOH); ${ }^{1} \mathrm{H}$ NMR $\left(500 \mathrm{MHz}, \mathrm{CD}_{3} \mathrm{OD}\right) \delta 8.37(\mathrm{~d}, 3.3$ $\mathrm{Hz}, 1 \mathrm{H}), 8.07(\mathrm{~s}, 1 \mathrm{H}), 7.63(\mathrm{~d}, 1.7 \mathrm{~Hz}, 1 \mathrm{H}), 7.52(\mathrm{~d}, 1.8 \mathrm{~Hz}, 1 \mathrm{H}), 7.49(\mathrm{~d}, 8.6 \mathrm{~Hz}, 1 \mathrm{H}), 7.46\left(\mathrm{AA}^{\prime} \mathrm{BB}^{\prime} \mathrm{X}, J_{\mathrm{AB}}\right.$ $\left.=8.9, J_{\mathrm{AF}}=5.3 \mathrm{~Hz}, 2 \mathrm{H}\right), 7.14\left(\mathrm{AA}^{\prime} \mathrm{BB}^{\prime} \mathrm{X}, J_{\mathrm{AB}}=J_{\mathrm{BF}}=8.8 \mathrm{~Hz}, 2 \mathrm{H}\right), 7.00(\mathrm{~d}, 4.6 \mathrm{~Hz}, 1 \mathrm{H}), 6.61(\mathrm{~d}, 2.4 \mathrm{~Hz}$, $1 \mathrm{H}), 6.57(\mathrm{dd}, 8.5,2.4 \mathrm{~Hz}, 1 \mathrm{H}), 4.72(\mathrm{~d}, 6.3 \mathrm{~Hz}, 2 \mathrm{H}), 4.26(\mathrm{td}, 3.8,1.6 \mathrm{~Hz}, 1 \mathrm{H}), 4.13(\mathrm{dd}, 8.8,3.9 \mathrm{~Hz}, 1 \mathrm{H})$, 3.96-3.88 (overlapping signals: $\mathrm{m}, 1 \mathrm{H}$ and 3.90, s, 3H), $3.82(\mathrm{~s}, 3 \mathrm{H}), 3.49(\mathrm{dd}, 12.5,3.9 \mathrm{~Hz}, 1 \mathrm{H}), 3.33-3.22$ (overlapping signals: $3.29, \mathrm{sp}, 7.0 \mathrm{~Hz}, 1 \mathrm{H}$ and 3.24 , dd, 12.6, $1.7 \mathrm{~Hz}, 1 \mathrm{H}), 1.39(\mathrm{~d}, 7.0 \mathrm{~Hz}, 6 \mathrm{H}) ;{ }^{19} \mathrm{~F} \mathrm{NMR}$ (470 MHz, CD 3 OD) $\delta-77.01,-112.77 ;{ }^{13} \mathrm{C}$ NMR $\left(125 \mathrm{MHz}, \mathrm{CD}_{3} \mathrm{OD}\right) \delta 177.05,166.17 / 164.19(\mathrm{~d}, 257.6$ Hz), 163.13, 161.47, 161.33, 158.83, 153.53, 149.15, 142.83, 131.86/131.79 (d, 8.3 Hz), 130.25, 129.25, $126.14,125.69,123.30,122.30,117.22,117.00,116.83,116.60,116.15,113.51,106.38,99.87,74.81,70.80$, 63.74, 55.91, 51.11, 48.69, 27.98, 21.25; HRMS (ESI) $m / z$ calcd for $\mathrm{C}_{35} \mathrm{H}_{36} \mathrm{FN}_{5} \mathrm{O}_{6} \mathrm{H}^{+} 642.2722$, found 642.2735. Anal. calcd for $\mathrm{C}_{35} \mathrm{H}_{36} \mathrm{FN}_{5} \mathrm{O}_{6} \cdot 2.3\left(\mathrm{CF}_{3} \mathrm{COOH}\right): \mathrm{C}, 52.62 ; \mathrm{H}, 4.27 ; \mathrm{N}, 7.75$. Found $\mathrm{C}, 52.61 ; \mathrm{H}$, $4.44 ; \mathrm{N}, 7.73$.

$(1 R, 2 S, 10 a R)-N-\{4-[3-(4-F l u o r o p h e n y l)-5-i s o p r o p y l i s o x a z o l-4-y l] p y r i d i n-2-y l\}-1,2-d i h y d r o x y-6-(4-$ methoxyphenyl)-2,3,10,10a-tetrahydro-1H,5H-dipyrrolo[1,2-a:1',2'-d]pyrazine-8-carboxamide (31a). To a solution of $29 \mathrm{~d}(20 \mathrm{mg}, 0.043 \mathrm{mmol})$ in methanol $(2 \mathrm{~mL})$ were added 2 drops of formalin (formaldehyde solution, 37\% in water). After being stirred at room temperature for $3 \mathrm{~h}$ the reaction was concentrated, and the crude product purified by automated column chromatography (silica gel, 
methanol/dichloromethane $0-10 \%)$ to afford $9 \mathrm{mg}$ of 31a as a colorless solid. ${ }^{1} \mathrm{H}$ NMR $(400 \mathrm{MHz}$, $d_{6-\mathrm{DMSO}} \delta 10.39(\mathrm{~s}, 1 \mathrm{H}, \mathrm{NH}), 8.36(\mathrm{~d}, 4.6 \mathrm{~Hz}, 1 \mathrm{H}), 8.10(\mathrm{~s}, 1 \mathrm{H}), 7.57(\mathrm{~s}, 1 \mathrm{H}), 7.46\left(\mathrm{AA}^{\prime} \mathrm{BB}^{\prime} \mathrm{X}, J_{\mathrm{AB}}=\right.$ $\left.8.9, J_{\mathrm{AF}}=5.5 \mathrm{~Hz}, 2 \mathrm{H}\right), 7.30\left(\mathrm{AA}^{\prime} \mathrm{XX} X^{\prime}, J_{\mathrm{AX}}=8.7 \mathrm{~Hz}, 2 \mathrm{H}\right), 7.27\left(\mathrm{AA}^{\prime} \mathrm{BB}^{\prime} \mathrm{X}, J_{\mathrm{AB}}=J_{\mathrm{BF}}=8.9 \mathrm{~Hz}, 2 \mathrm{H}\right), 6.98$ $\left(\mathrm{AA}^{\prime} \mathrm{XX}^{\prime}, J_{\mathrm{AX}}=8.8 \mathrm{~Hz}, 2 \mathrm{H}\right), 6.89(\mathrm{dd}, 5.1,1.5 \mathrm{~Hz}, 1 \mathrm{H}), 4.95-4.84$ (overlapping signals: 4.93, dd, 13.0, 3.8 $\left.\mathrm{Hz}, 1 \mathrm{H} ; 4.93, \mathrm{~s}_{\mathrm{br}}, 1 \mathrm{H}, \mathrm{OH}\right), 4.84\left(\mathrm{~s}_{\mathrm{br}}, 1 \mathrm{H}, \mathrm{OH}\right), 4.10(\mathrm{~d}, 14.7 \mathrm{~Hz}, 1 \mathrm{H}), 4.09-4.03(\mathrm{~m}, 1 \mathrm{H}), 3.80-3.74(\mathrm{~m}$, $1 \mathrm{H} ; 3.77, \mathrm{~s}, 3 \mathrm{H}), 3.66(\mathrm{~m}, 1 \mathrm{H}), 3.58(\mathrm{~d}, 14.5 \mathrm{~Hz}, 1 \mathrm{H}), 3.47(\mathrm{dd}, 9.7,6.4 \mathrm{~Hz}, 1 \mathrm{H}), 3.22(\mathrm{sp}, 7.0 \mathrm{~Hz}, 1 \mathrm{H})$, 2.60-2.54 (m, 1H), 2.29-2.25 (m, 1H), $1.32(\mathrm{~d}, 6.9 \mathrm{~Hz}, 6 \mathrm{H}) ;{ }^{19} \mathrm{~F}$ NMR (376 MHz, $d_{6-\mathrm{DMSO}} \delta-111.04$; ${ }^{13} \mathrm{C}$ NMR (125 MHz, $d_{6}$-DMSO) $\delta 174.80,164.0 / 161.6$ (d, $\left.247.3 \mathrm{~Hz}\right), 159.80,159.58,157.36,152.97$, 148.30, 139.42, 130.45/130.36 (d, 8.6 Hz), 130.1, 127.74, 127.23, 124.56/124.54 (d, 3.2 Hz), 122.83, 119.87, $118.34,116.03 / 115.81$ (d, $21.9 \mathrm{~Hz}), 114.49,114.20,114.08,112.13,73.99,67.99,63.13,61.21,55.00,50.64$ (br), 49.79 (br), 26.00, 20.60; HRMS (ESI) $m / z$ calcd for $\mathrm{C}_{35} \mathrm{H}_{34} \mathrm{FN}_{5} \mathrm{O}_{5} \mathrm{H}^{+}$624.2617, found 624.2613.

(1S,2R,10aS)-N-\{4-[3-(4-Fluorophenyl)-5-isopropylisoxazol-4-yl]pyridin-2-yl\}-1,2-dihydroxy-6-(4methoxyphenyl)-2,3,10,10a-tetrahydro-1H,5H-dipyrrolo[1,2-a:1',2'-d]pyrazine-8-carboxamide

Employing the same procedure as for the preparation of 31a compound $29 \mathbf{e}(31 \mathrm{mg}, 0.043 \mathrm{mmol})$ was dissolved in methanol $(2 \mathrm{~mL}$ ) and treated with 2 drops of formalin (formaldehyde solution, $37 \%$ in water) at room temperature overnight to afford $30 \mathrm{mg}$ of $\mathbf{3 1 b}$ as a light-yellow solid. All spectroscopic data of $\mathbf{3 1 b}$ was consistent with that recorded for 31a. HRMS (ESI) $\mathrm{m} / z$ calcd for $\mathrm{C}_{35} \mathrm{H}_{34} \mathrm{FN}_{5} \mathrm{O}_{5} \mathrm{H}^{+}$ 624.2617, found 624.2615 .

\subsection{Plasmids, Overexpression and Purification of Glutathion S-Transferase Fusion CK1 1 TV1 Protein}

Human CK1 $1 \delta$ transcription variant 1 (CK1ס TV1_NM_001893.4) has been amplified from the cDNA obtained from HT1080 cell line, using CK1ס_forward-primer: 5'-GGA TCC ATG GAG CTG AGA GTC GGG AAC AG-3' and CK1 1 _reverse primer: 5'-GGA TCC TCA TCG GTG CAC GAC AGA CTG A-3'. The CK1 $\delta$ DNA construct was cloned into pSC-A cloning vector (Agilent technologies, Munich, Germany) with BamHI enzyme, to generate plasmids pGEX6-P3-GST-CK1 1 TV1 (FP1417). Overexpression and purification of GST-CK1 $1 \delta$ TV1 protein has been performed as described before [87].

\subsection{Kinase Assays}

In vitro kinase assays have been performed to both screen, as well as to calculate $\mathrm{IC}_{50}$ values of the compounds for $\mathrm{CK} 1 \delta$ and $\varepsilon$. Each reaction was carried out using $2 \mu \mathrm{Ci}{ }^{32} \mathrm{P}-\gamma$-ATP in kinase buffer containing $25 \mathrm{mM}$ Tris- $\mathrm{HCl}(\mathrm{pH}=7.5), 10 \mathrm{mM} \mathrm{MgCl}_{2}, 100 \mu \mathrm{M}$ EDTA, and $10 \mu \mathrm{M}$ ATP. Potential inhibitor compounds were used in a concentration of $10 \mu \mathrm{M}$ for screening assays and in a dilution series ranging from $10 \mu \mathrm{M}$ to $5 \mathrm{nM}$ final reaction concentration, which was prepared by serial dilution in DMSO, in order to calculate the $\mathrm{IC}_{50}$ values. Recombinant human CK1 $1 \delta$ transcription variant 1 (expressed and purified as GST fusion protein as described earlier) and human GST-CK1E (PV3500; Invitrogen) were used as sources of enzyme, while $\alpha$-casein (C6780; Sigma-Aldrich) was used as substrate. Kinase reactions were incubated for $30 \mathrm{~min}$ at $30^{\circ} \mathrm{C}$ and then stopped with loading dye (50 $\mathrm{mM}$ Tris- $\mathrm{HCl}(\mathrm{pH}=6.8), 5 \%(v / v) \beta$-mercaptoethanol $(\mathrm{MSH}), 10 \%(v / v)$ glycerol, $2 \%(w / v)$ sodium dodecyl sulfate (SDS), $0.1 \%(w / v)$ bromphenol blue). Subsequently, reactions were separated by sodium dodecyl sulfate polyacrylamide gel electrophoresis (SDS-PAGE) and phosphorylated protein bands were visualized on dried gels by autoradiography. The phosphorylated substrate protein bands were excised, and phosphorylation was quantified by Cherenkov counting. Dose-response analyses and calculation of $\mathrm{IC}_{50}$ values were carried out using GraphPad Prism 6 statistical software (San Diego, CA, USA).

\subsection{Cell Culture}

\subsubsection{Cell Lines}

The human colon adenocarcinoma cell line HT-29 was grown in McCoy's 5A medium, whereas MCF7 cells were grown in DMEM medium. All media were supplemented with $10 \%$ fetal calf serum 
(FCS), 100 units $/ \mathrm{mL}$ penicillin, $100 \mu \mathrm{g} / \mathrm{mL}$ streptomycin and $2 \mathrm{mM}$ glutamine. All cells were grown at $37^{\circ} \mathrm{C}$ in a humidified $5 \%$ carbon dioxide atmosphere.

\subsubsection{Cellular Assays and $\mathrm{EC}_{50}$ Determination}

In order to analyze the effects of selected inhibitors on the cell viability of HT-29 and MCF7 cells, MTT assays were performed. $1 \times 10^{4}$ cells/well were seeded in 96-well cell culture plates. After $24 \mathrm{~h}$ cells were treated with increasing concentrations of the indicated inhibitor compounds, with untreated and DMSO-treated cells as control. After an incubation period of $48 \mathrm{~h}, 10 \mu \mathrm{L}$ of MTT solution $(5 \mathrm{mg} / \mathrm{mL}$ in PBS) were added and cells were incubated for $4 \mathrm{~h}$. MTT-containing medium was carefully removed and $100 \mu \mathrm{L}$ acidic isopropanol $(0.04 \mathrm{~N} \mathrm{HCl}$ in isopropanol) per well were added. To dissolve formazan crystals, plates were shaken for $30 \mathrm{~min}$ on an orbital shaker in the dark. Finally, dissolved crystals were measured spectrophotometrically at $570 \mathrm{~nm}$. All experiments were performed in triplicate with four technical replicates per assay. Results were normalized considering the mean optical density value of control wells as $100 \%$. GraphPad Prism 6 software (San Diego, CA, USA) was used to calculate $\mathrm{EC}_{50}$ values.

\subsection{Macromolecular Crystallography}

For structural studies, $\mathrm{His}_{6}$-tagged tCK1 $\delta$ was recombinantly expressed in BL21 (DE3) TaKaRa 2 Escherichia coli cells (Clontech) and afterwards purified by immobilized metal affinity chromatography as described previously [40].

For co-crystallization of tCK1 $1 \delta$ with $29 \mathrm{~d}$ and $29 \mathrm{e}$, protein stock solution $\left(10 \mathrm{mg} \cdot \mathrm{mL}^{-1}\right)$ was mixed 30:1 with $10 \mathrm{mM}$ of the respective compounds (solubilized in DMSO) and incubated for $30 \mathrm{~min}$ at room temperature. Sitting drop crystallization trials were set up at room temperature with drop ratios of $3 \mu \mathrm{L}$ protein/inhibitor solution to $2 \mu \mathrm{L}$ precipitant solution.

Crystals appeared after three to seven days in drops containing 0.1 M MES ( $\mathrm{pH}=5.5), 10 \%$ $(w / v)$ PEG 4000 and $0.2 \mathrm{M}$ lithium sulfate. For data collection, these crystals were cryo-protected by swiping them though reservoir solution supplemented with $25 \%(v / v)$ glycerol and subsequently flash frozen. Diffraction data were collected at beamline P13 at the PETRA/EMBL Hamburg, German Synchrotron Research Centre (DESY) campus, Hamburg, Germany (tCK1 $\delta$ with 29d) and at beamline X06DA at the Swiss Light Source, Paul-Scherrer- Institute, Villigen, Switzerland. XDS [87] was used for data processing and the structures were solved by molecular replacement using the program PHASER [88,89] with a truncated crystal structure of CK1ס (pdb 4TWC [72]) as a search model.

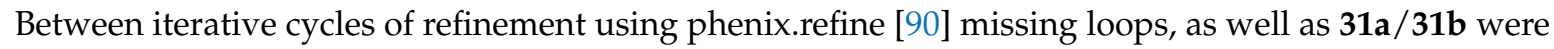
manually built with Coot [91]. Restraints of 31a and 31b were calculated using phenix.elbow [92].

\section{Conclusions}

By using isoxazole-based CK1 $\delta$ inhibitor 8 as model compound we implemented chiral pyrrolidine scaffolds to investigate specific interactions within the ATP binding pocket of this kinase. A synthetic approach leading to stereochemically defined compounds $(\mathbf{2 9 d}-\mathbf{f})$ was established. Biological evaluation proved the compounds to be quite potent in in vitro CK1 kinase assays, but revealed only a minor impact of the different stereoisomers towards affinity differences and CK1 isoform-selectivity. However, selectivity profiling in a panel of 320 kinases showed compound $29 \mathrm{~d}$ to be relatively specific for CK1. Compounds $\mathbf{2 9 d}-\mathbf{f}$ showed also modest effects on HT-29 and MCF-7 cell lines. Surprisingly, X-ray crystallographic data revealed new cyclized compounds within the analyzed protein-ligand complexes, which were supposedly produced by spontaneous Pictet-Spengler cyclization implementing one equivalent formaldehyde during sample soaking procedures. In fact, this hypothesis could be confirmed by reacting the original compounds $29 \mathrm{~d} / \mathbf{e}$ with formaldehyde to yield the new ligands $31 \mathrm{a} / \mathbf{b}$. Taken together, functionalized pyrrolidines are expedient chiral scaffolds in medicinal chemistry and may have a potential for the development of potent and selective kinase inhibitors targeting the ribose pocket of the ATP binding site. 
Supplementary Materials: The following are available online, Figure S1: X-Ray crystal structure of compound 25d Table S1: Data collection, structure refinement and Ramachrandran plot results of protein crystallization, Table S2: Selectivity profile of compound 29d, Appendix: NMR spectra, HPLC chromatograms and DSC traces.

Author Contributions: A.L., C.P., U.K. and U.B. conceived and designed the experiments; A.L., D.S. and T.v.D. performed synthesis; C.I. and M.K. performed biological testings, C.P. and E.B. performed crystallization experiments, C.P. and G.J.G. performed X-ray crystallography, A.L., D.S., C.I., M.K. and C.P. analyzed the data; D.S. and A.L. wrote the paper.

Funding: Funding for the synchrotron visit has been provided by the iNEXT initiative (proposal 1859, EU program Horizon 2020).

Acknowledgments: A.L. thanks New Zealand Pharmaceutical Ltd. (NZP) and the Ministry of Business, Innovation and Employment (MBIE; C08X0711) for funding and Bruce Hamilton for invaluable assistance with HPLC analysis. We thank the staff at the beamlines X06SA and X06DA at the Swiss Light Source, Paul-Scherrer-Institute, Villigen, Switzerland, as well as the beam line staff from PETRA III, DESY Hamburg, for support during synchrotron data collection.

Conflicts of Interest: The authors declare no competing financial interest.

\section{References}

1. Roughley, S.D.; Jordan, A.M. The Medicinal Chemist's Toolbox: An Analysis of Reactions Used in the Pursuit of Drug Candidates. J. Med. Chem. 2011, 54, 3451-3479. [CrossRef] [PubMed]

2. Lovering, F.; Bikker, J.; Humblet, C. Escape from Flatland: Increasing Saturation as an Approach to Improving Clinical Success. J. Med. Chem. 2009, 52, 6752-6756. [CrossRef] [PubMed]

3. Walters, W.P.; Green, J.; Weiss, J.R.; Murcko, M.A. What Do Medicinal Chemists Actually Make? A 50-Year Retrospective. J. Med. Chem. 2011, 54, 6405-6416. [CrossRef] [PubMed]

4. Goldberg, F.W.; Kettle, J.G.; Kogej, T.; Perry, M.W.D.; Tomkinson, N.P. Designing novel building blocks is an overlooked strategy to improve compound quality. Drug Discov. Today 2015, 20, 11-17. [CrossRef] [PubMed]

5. Zhang, J.; Yang, P.L.; Gray, N.S. Targeting cancer with small molecule kinase inhibitors. Nat. Rev. Cancer 2009, 9, 28-39. [CrossRef] [PubMed]

6. Traxler, P.; Furet, P. Strategies toward the Design of Novel and Selective Protein Tyrosine Kinase Inhibitors. Pharmacol. Ther. 1999, 82, 195-206. [CrossRef]

7. Cheung, J.; Ginter, C.; Cassidy, M.; Franklin, M.C.; Rudolph, M.J.; Robine, N.; Darnell, R.B.; Hendrickson, W.A. Structural insights into mis-regulation of protein kinase A in human tumors. Proc. Natl. Acad. Sci. USA 2015, 112, 1374-1379. [CrossRef] [PubMed]

8. Hafenbradl, D.; Baumann, M.; Neumann, L. In Vitro Characterization of Small-Molecule Kinase Inhibitors. In Protein Kinases as Drug Targets, 1st ed.; Klebl, B., Müller, G., Hamacher, M., Eds.; Wiley-VCH: Weinheim, Germany, 2011; pp. 26-27, ISBN 978-3-527-31790-5.

9. Laufer, S.; Margutti, S.; Hauser, D. Medicinal Chemistry Approaches for the Inhibition of the p38 MAPK Pathway. In Protein Kinases as Drug Targets, 1st ed.; Klebl, B., Müller, G., Hamacher, M., Eds.; Wiley-VCH: Weinheim, Germany, 2011; pp. 282-286, ISBN 978-3-527-31790-5.

10. Adams, J.L.; Boehm, J.C.; Gallagher, T.F.; Kassis, S.; Webb, E.F.; Hall, R.; Sorenson, M.; Garigipati, R.; Griswold, D.E.; Lee, J.C. Pyrimidinylimidazole inhibitors of p38: Cyclic N-1 imidazole substituents enhance p38 kinase inhibition and oral activity. Bioorg. Med. Chem. Lett. 2001, 11, 2867-2870. [CrossRef]

11. Hennequin, L.F.; Allen, J.; Breed, J.; Curwen, J.; Fennell, M.; Green, T.P.; Lambert-van der Brempt, C.; Morgentin, R.; Norman, R.A.; Olivier, A.; et al. N-(5-Chloro-1,3-benzodioxol-4-yl)-7-[2-(4-methylpiperazin-1yl)ethoxy]-5- (tetrahydro-2H-pyran-4-yloxy)quinazolin-4-amine, a Novel, Highly Selective, Orally Available, Dual-Specific c-Src/Abl Kinase Inhibitor. J. Med. Chem. 2006, 49, 6465-6488. [CrossRef] [PubMed]

12. Caldwell, J.J.; Welsh, E.J.; Matijssen, C.; Anderson, V.E.; Antoni, L.; Boxall, K.; Urban, F.; Hayes, A.; Raynaud, F.I.; Rigoreau, L.J.M.; et al. Structure-Based Design of Potent and Selective 2-(Quinazolin-2-yl)phenol Inhibitors of Checkpoint Kinase 2. J. Med. Chem. 2011, 54, 580-590. [CrossRef] [PubMed]

13. Ballard, P.; Bradbury, R.H.; Harris, C.S.; Hennequin, L.F.A.; Hickinson, M.; Johnson, P.D.; Kettle, J.G.; Klinowska, T.; Leach, A.G.; Morgentin, R.; et al. Inhibitors of epidermal growth factor receptor tyrosine kinase: Novel C-5 substituted anilinoquinazolines designed to target the ribose pocket. Bioorg. Med. Chem. Lett. 2006, 16, 1633-1637. [CrossRef] [PubMed] 
14. Jiang, J.K.; Shen, M.; Thomas, C.J.; Boxer, M.B. Chiral Kinase Inhibitors. Curr. Top. Med. Chem. 2011, 11, 800-809. [CrossRef] [PubMed]

15. Buhler, S.; Goettert, M.; Schollmeyer, D.; Albrecht, W.; Laufer, S.A. Chiral Sulfoxides as Metabolites of 2-Thioimidazole-Based p38 $\alpha$ Mitogen-Activated Protein Kinase Inhibitors: Enantioselective Synthesis and Biological Evaluation. J. Med. Chem. 2011, 54, 3283-3297. [CrossRef] [PubMed]

16. Koch, P.; Bäuerlein, C.; Jank, H.; Laufer, S. Targeting the Ribose and Phosphate Binding Site of p38 Mitogen-Activated Protein (MAP) Kinase: Synthesis and Biological Testing of 2-Alkylsulfanyl-, 4(5)-Aryl-, 5(4)-Heteroaryl-Substituted Imidazoles. J. Med. Chem. 2008, 51, 5630-5640. [CrossRef] [PubMed]

17. McElroy, W.T.; Michael Seganish, W.; Jason Herr, R.; Harding, J.; Yang, J.; Yet, L.; Komanduri, V.; Prakash, K.C.; Lavey, B.; Tulshian, D.; et al. Discovery and hit-to-lead optimization of 2,6-diaminopyrimidine inhibitors of interleukin-1 receptor-associated kinase 4. Bioorg. Med. Chem. Lett. 2015, 25, 1836-1841. [CrossRef] [PubMed]

18. Lee, K.L.; Ambler, C.M.; Anderson, D.R.; Boscoe, B.P.; Bree, A.G.; Brodfuehrer, J.I.; Chang, J.S.; Choi, C.; Chung, S.; Curran, K.J.; et al. Discovery of Clinical Candidate 1-\{[(2S,3S,4S)-3-Ethyl-4-fluoro-5-oxopyrrolidin-2-yl]methoxy\}-7-methoxyisoquinoline-6-carboxamide (PF-06650833), a Potent, Selective Inhibitor of Interleukin-1 Receptor Associated Kinase 4 (IRAK4), by Fragment-Based Drug Design. J. Med. Chem. 2017, 60, 5521-5542. [CrossRef] [PubMed]

19. Gani, O.; Engh, R. Protein kinase inhibition of clinically important staurosporine analogues. Nat. Prod. Rep. 2010, 27, 489-498. [CrossRef] [PubMed]

20. Kinoshita, T.; Matsubara, M.; Ishiguro, H.; Okita, K.; Tada, T. Structure of human Fyn kinase domain complexed with staurosporine. Biochem. Biophys. Res. Commun. 2006, 346, 840-844. [CrossRef] [PubMed]

21. Jin, L.; Pluskey, S.; Petrella, E.C.; Cantin, S.M.; Gorga, J.C.; Rynkiewicz, M.J.; Pandey, P.; Strickler, J.E.; Babine, R.E.; Weaver, D.T.; et al. The three-dimensional structure of the ZAP-70 kinase domain in complex with staurosporine: Implications for the design of selective inhibitors. J. Biol. Chem. 2004, 279, 42818-42825. [CrossRef] [PubMed]

22. Bunkoczi, G.; Salah, E.; Filippakopoulos, P.; Fedorov, O.; Müller, S.; Sobott, F.; Parker, S.A.; Zhang, H.; Min, W.; Turk, B.E.; et al. Structural and Functional Characterization of the Human Protein Kinase ASK1. Structure 2007, 15, 1215-1226. [CrossRef] [PubMed]

23. Ruegg, U.T.; Burgess, G.M. Staurosporine, K-252 and UCN-01: Potent but nonspecific inhibitors of protein kinases. Trends Pharmacol. Sci. 1989, 10, 218-220. [CrossRef]

24. Sanchez, C.; Salas, A.P.; Brana, A.F.; Palomino, M.; Pineda-Lucena, A.; Carbajo, R.J.; Mendez, C.; Moris, F.; Salas, J.A. Generation of potent and selective kinase inhibitors by combinatorial biosynthesis of glycosylated indolocarbazoles. Chem. Commun. 2009, 4118-4120. [CrossRef] [PubMed]

25. Al-awar, R.S.; Ray, J.E.; Hecker, K.A.; Huang, J.; Waid, P.P.; Shih, C.; Brooks, H.B.; Spencer, C.D.; Watkins, S.A.; Patel, B.R.; et al. 1,7-annulated indolocarbazoles as cyclin-dependent kinase inhibitors. Bioorg. Med. Chem. Lett. 2004, 14, 3217-3220. [CrossRef] [PubMed]

26. Zimmermann, A.; Wilts, H.; Lenhardt, M.; Hahn, M.; Mertens, T. Indolocarbazoles exhibit strong antiviral activity against human cytomegalovirus and are potent inhibitors of the pUL97 protein kinase. Antivir. Res. 2000, 48, 49-60. [CrossRef]

27. Mullard, A. FDA approves first new ALS drug in over 20 years. Nat. Rev. Drug Discov. 2017, 16, 375. [CrossRef]

28. Manley, P.W.; Weisberg, E.; Sattler, M.; Griffin, J.D. Midostaurin, a Natural Product-Derived Kinase Inhibitor Recently Approved for the Treatment of Hematological Malignancies. Biochemistry 2017, 57, 477-478. [CrossRef] [PubMed]

29. Peifer, C.; Abadleh, M.; Bischof, J.; Hauser, D.; Schattel, V.; Hirner, H.; Knippschild, U.; Laufer, S. 3,4-Diaryl-isoxazoles and -imidazoles as Potent Dual Inhibitors of p38 $\alpha$ Mitogen Activated Protein Kinase and Casein Kinase 18. J. Med. Chem. 2009, 52, 7618-7630. [CrossRef] [PubMed]

30. Knippschild, U.; Krüger, M.; Richter, J.; Xu, P.; García-Reyes, B.; Peifer, C.; Halekotte, J.; Bakulev, V.; Bischof, J. The CK1 family: Contribution to cellular stress response and its role in carcinogenesis. Front. Oncol. 2014, 4, 96. [CrossRef] [PubMed]

31. Knippschild, U.; Gocht, A.; Wolff, S.; Huber, N.; Löhler, J.; Stöter, M. The casein kinase 1 family: Participation in multiple cellular processes in eukaryotes. Cell. Signal. 2005, 17, 675-689. [CrossRef] [PubMed] 
32. Knippschild, U.; Wolff, S.; Giamas, G.; Brockschmidt, C.; Wittau, M.; Würl, P.U.; Eismann, T.; Stöter, M. The Role of the Casein Kinase 1 (CK1) Family in Different Signaling Pathways Linked to Cancer Development. Oncol. Res. Treat. 2005, 28, 508-514. [CrossRef] [PubMed]

33. Yasojima, K.; Kuret, J.; DeMaggio, A.J.; McGeer, E.; McGeer, P.L. Casein kinase 1 delta mRNA is upregulated in Alzheimer disease brain. Brain Res. 2000, 865, 116-120. [CrossRef]

34. Horne, G.; Wilson, F.X.; Tinsley, J.; Williams, D.H.; Storer, R. Iminosugars past, present and future: Medicines for tomorrow. Drug Discov. Today 2011, 16, 107-118. [CrossRef] [PubMed]

35. Evans, G.B. The Synthesis of N-Ribosyl Transferase Inhibitors Based on a Transition State Blueprint. Aust. J. Chem. 2004, 57, 837-854. [CrossRef]

36. Winchester, B.G. Iminosugars: From botanical curiosities to licensed drugs. Tetrahedron Asymmetry 2009, 20, 645-651. [CrossRef]

37. Hernández, D.; Boto, A. Nucleoside Analogues: Synthesis and Biological Properties of Azanucleoside Derivatives. Eur. J. Org. Chem. 2014, 11, 2201-2220. [CrossRef]

38. Cipolla, L.; La Ferla, B.; Airoldi, C.; Zona, C.; Orsato, A.; Shaikh, N.; Russo, L.; Nicotra, F. Carbohydrate mimetics and scaffolds: Sweet spots in medicinal chemistry. Future Med. Chem. 2010, 2, 587-599. [CrossRef] [PubMed]

39. Fischer, S.; Koeberle, S.C.; Laufer, S.A. p38 $\alpha$ mitogen-activated protein kinase inhibitors, a patent review (2005-2011). Expert Opin. Ther. Pat. 2011, 21, 1843-1866. [CrossRef] [PubMed]

40. Halekotte, J.; Witt, L.; Ianes, C.; Kruger, M.; Buhrmann, M.; Rauh, D.; Pichlo, C.; Brunstein, E.; Luxenburger, A.; Baumann, U.; et al. Optimized 4,5-Diarylimidazoles as Potent/Selective Inhibitors of Protein Kinase CK1 $\delta$ and Their Structural Relation to p38 $\alpha$ MAPK. Molecules 2017, 22, 522. [CrossRef] [PubMed]

41. Long, A.; Zhao, H.; Huang, X. Structural Basis for the Interaction between Casein Kinase 1 Delta and a Potent and Selective Inhibitor. J. Med. Chem. 2012, 55, 956-960. [CrossRef] [PubMed]

42. Xu, R.M.; Carmel, G.; Sweet, R.M.; Kuret, J.; Cheng, X. Crystal structure of casein kinase-1, a phosphate-directed protein kinase. EMBO J. 1995, 14, 1015-1023. [CrossRef] [PubMed]

43. Longenecker, K.L.; Roach, P.J.; Hurley, T.D. Three-dimensional Structure of Mammalian Casein Kinase I: Molecular Basis for Phosphate Recognition. J. Mol. Biol. 1996, 257, 618-631. [CrossRef] [PubMed]

44. Babu, Y.S.; Kotian, P.L.; Bantia, S.; Wu, M.; Kumar, V.S. Antiviral Azasugar-Containing Nucleosides. WO 2014/078778 A2, 22 May 2014.

45. Kotian, P.L.; Babu, Y.S. Compositions and Methods for Inhibiting Viral Polymerase. WO 2013/158746 A1, 24 October 2013.

46. Clinch, K.; Evans, G.B.; Fleet, G.W.J.; Furneaux, R.H.; Johnson, S.W.; Lenz, D.H.; Mee, S.P.H.; Rands, P.R.; Schramm, V.L.; Taylor Ringia, E.A.; et al. Syntheses and bio-activities of the L-enantiomers of two potent transition state analogue inhibitors of purine nucleoside phosphorylases. Org. Biomol. Chem. 2006, 4, 1131-1139. [CrossRef] [PubMed]

47. Malladi, V.L.A.; Sobczak, A.J.; Maricic, N.; Murugapiran, S.K.; Schneper, L.; Makemson, J.; Mathee, K.; Wnuk, S.F. Substituted lactam and cyclic azahemiacetals modulate Pseudomonas aeruginosa quorum sensing. Bioorg. Med. Chem. 2011, 19, 5500-5506. [CrossRef] [PubMed]

48. Malladi, V.L.A.; Sobczak, A.J.; Meyer, T.M.; Pei, D.; Wnuk, S.F. Inhibition of LuxS by S-ribosylhomocysteine analogues containing a [4-aza]ribose ring. Bioorg. Med. Chem. 2011, 19, 5507-5519. [CrossRef] [PubMed]

49. Malladi, V.L.; Schneper, L.; Sobczak, A.J.; Mathee, K.; Wnuk, S.F. 2-methylthiopyrrolidines and Their Use for Modulating Bacterial Quorum Sensing. WO 2012/174511 A1, 18 June 2012.

50. Maity, P.; Zabel, M.; König, B. Tetrahydrofuran C $\alpha$-Tetrasubstituted Amino Acids: Two Consecutive $\beta$-Turns in a Crystalline Linear Tripeptide. J. Org. Chem. 2007, 72, 8046-8053. [CrossRef] [PubMed]

51. Keyes, R.F.; Carter, J.J.; Zhang, X.; Ma, Z. Short and Efficient Synthesis of a Vinyl-Substituted Tricyclic Erythromycin Derivative. Org. Lett. 2005, 7, 847-849. [CrossRef] [PubMed]

52. Krebs, A.; Ludwig, V.; Pfizer, J.; Dürner, G.; Göbel, M.W. Enantioselective Synthesis of Non-Natural Aromatic $\alpha$-Amino Acids. Chem. Eur. J. 2004, 10, 544-553. [CrossRef] [PubMed]

53. Aurelio, L.; Box, J.S.; Brownlee, R.T.C.; Hughes, A.B.; Sleebs, M.M. An Efficient Synthesis of N-Methyl Amino Acids by Way of Intermediate 5-Oxazolidinones. J. Org. Chem. 2003, 68, 2652-2667. [CrossRef] [PubMed] 
54. Webber, S.E.; Okano, K.; Little, T.L.; Reich, S.H.; Xin, Y.; Fuhrman, S.A.; Matthews, D.A.; Love, R.A.; Hendrickson, T.F.; Patick, A.K.; et al. Tripeptide Aldehyde Inhibitors of Human Rhinovirus 3C Protease: Design, Synthesis, Biological Evaluation, and Cocrystal Structure Solution of $\mathrm{P}_{1}$ Glutamine Isosteric Replacements. J. Med. Chem. 1998, 41, 2786-2805. [CrossRef] [PubMed]

55. Brackmann, F.; Colombo, N.; Cabrele, C.; de Meijere, A. An Improved Synthesis of 3,4-(Aminomethano)proline and Its Incorporation into Small Oligopeptides. Eur. J. Org. Chem. 2006, 4440-4450. [CrossRef]

56. Takahata, H.; Banba, Y.; Ouchi, H.; Nemoto, H.; Kato, A.; Adachi, I. Asymmetric Synthesis of the Four Possible Fagomine Isomers. J. Org. Chem. 2003, 68, 3603-3607. [CrossRef] [PubMed]

57. Murruzzu, C.; Riera, A. Enantioselective synthesis of hydroxylated pyrrolidines via Sharpless epoxidation and olefin metathesis. Tetrahedron Asymmetry 2007, 18, 149-154. [CrossRef]

58. Qiu, X.L.; Qing, F.L. Synthesis of 3'-Deoxy-3'-difluoromethyl Azanucleosides from trans-4-Hydroxy-L-proline. J. Org. Chem. 2005, 70, 3826-3837. [CrossRef] [PubMed]

59. Handy, S.T.; Zhang, Y.; Bregman, H. A Modular Synthesis of the Lamellarins: Total Synthesis of Lamellarin G Trimethyl Ether. J. Org. Chem. 2004, 69, 2362-2366. [CrossRef] [PubMed]

60. Smith, J.A.; Ng, S.; White, J. The regioselective synthesis of aryl pyrroles. Org. Biomol. Chem. 2006, 4, 2477-2482. [CrossRef] [PubMed]

61. Handy, S.T.; Bregman, H.; Lewis, J.; Zhang, X.; Zhang, Y. An unusual dehalogenation in the Suzuki coupling of 4-bromopyrrole-2-carboxylates. Tetrahedron Lett. 2003, 44, 427-430. [CrossRef]

62. Jadhav, P.K.; Krishnan, V.; Kim, E.J. Tetrahydrocyclopenta[b]indole Androgen Receptor Modulators. WO 2009/140448 A1, 19 November 2009.

63. Nicolai, S.; Piemontesi, C.; Waser, J. A Palladium-Catalyzed Aminoalkynylation Strategy towards Bicyclic Heterocycles: Synthesis of ( \pm )-Trachelanthamidine. Angew. Chem. Int. Ed. 2011, 50, 4680-4683. [CrossRef] [PubMed]

64. Liu, L.; Norman, M.H.; Lee, M.; Xi, N.; Siegmund, A.; Boezio, A.A.; Booker, S.; Choquette, D.; D'Angelo, N.D.; Germain, J.; et al. Structure-Based Design of Novel Class II c-Met Inhibitors: 2. SAR and Kinase Selectivity Profiles of the Pyrazolone Series. J. Med. Chem. 2012, 55, 1868-1897. [CrossRef] [PubMed]

65. Hirner, H.; Günes, C.; Bischof, J.; Wolff, S.; Grothey, A.; Kühl, M.; Oswald, F.; Wegwitz, F.; Bösl, M.; Trauzold, A.; et al. Impaired CK1 Delta Activity Attenuates SV40-Induced Cellular Transformation In Vitro and Mouse Mammary Carcinogenesis In Vivo. PLoS ONE 2012, 7, e29709. [CrossRef] [PubMed]

66. Richter, J.; Rudeck, S.; Kretz, A.-L.; Kramer, K.; Just, S.; Henne-Bruns, D.; Hillenbrand, A.; Leithäuser, F.; Lemke, J.; Knippschild, U. Decreased CK1 $\delta$ expression predicts prolonged survival in colorectal cancerpatients. Tumor Biol. 2016, 37, 8731-8739. [CrossRef] [PubMed]

67. Trainer, D.L.; Kline, T.; McCabe, F.L.; Faucette, L.F.; Feild, J.; Chaikin, M.; Anzano, M.; Reiman, D.; Hoffstein, S.; Li, D.-J.; et al. Biological characterization and oncogene expression in human colorectal carcinoma cell lines. Int. J. Cancer 1988, 41, 287-296. [CrossRef] [PubMed]

68. Huguet, E.L.; McMahon, J.A.; McMahon, A.P.; Bicknell, R.; Harris, A.L. Differential Expression of Human Wnt Genes 2, 3, 4, and 7B in Human Breast Cell Lines and Normal and Disease States of Human Breast Tissue. Cancer Res. 1994, 54, 2615-2621. [PubMed]

69. Schittek, B.; Sinnberg, T. Biological functions of casein kinase 1 isoforms and putative roles in tumorigenesis. Mol. Cancer 2014, 13, 231. [CrossRef] [PubMed]

70. Stöter, M.; Bamberger, A.-M.; Aslan, B.; Kurth, M.; Speidel, D.; Löning, T.; Frank, H.-G.; Kaufmann, P.; Löhler, J.; Henne-Bruns, D.; et al. Inhibition of casein kinase I delta alters mitotic spindle formation and induces apoptosis in trophoblast cells. Oncogene 2005, 24, 7964-7975. [CrossRef] [PubMed]

71. Richter, J.; Bischof, J.; Zaja, M.; Kohlhof, H.; Othersen, O.; Vitt, D.; Alscher, V.; Pospiech, I.; Garcia-Reyes, B.; Berg, S.; et al. Difluoro-dioxolo-benzoimidazol-benzamides as potent inhibitors of CK1 $1 \delta$ and $\varepsilon$ with nanomolar inhibitory activity on cancer cell proliferation. J. Med. Chem. 2014, 57, 7933-7946. [CrossRef] [PubMed]

72. Bischof, J.; Leban, J.; Zaja, M.; Grothey, A.; Radunsky, B.; Othersen, O.; Strobl, S.; Vitt, D.; Knippschild, U. 2-Benzamido- $N$-(1H-benzo[d]imidazol-2-yl)thiazole-4-carboxamide derivatives as potent inhibitors of CK1 $/ \varepsilon$. Amino Acids 2012, 43, 1577-1591. [CrossRef] [PubMed] 
73. Cheong, J.K.; Hung, N.T.; Wang, H.; Tan, P.; Voorhoeve, P.M.; Lee, S.H.; Virshup, D.M. IC261 induces cellcycle arrest and apoptosis of human cancer cells via CK1 $/ \varepsilon$ and $\mathrm{Wnt} / \beta$-catenin independent inhibition of mitotic spindle formation. Oncogene 2011, 30, 2558-2569. [CrossRef] [PubMed]

74. Pictet, A.; Spengler, T. Über die Bildung von Isochinolin-derivaten durch Einwirkung von Methylal auf Phenyl-äthylamin, Phenyl-alanin und Tyrosin. Ber. Dtsch. Chem. Ges. 1911, 44, 2030-2036. [CrossRef]

75. Stöckigt, J.; Antonchick, A.P.; Wu, F.; Waldmann, H. The Pictet-Spengler Reaction in Nature and in Organic Chemistry. Angew. Chem. Int. Ed. 2011, 50, 8538-8564. [CrossRef] [PubMed]

76. Rousseau, J.F.; Dodd, R.H. Synthesis of 3-Deaza- $\beta$-hydroxyhistidine Derivatives and Their Use for the Preparation of Substituted Pyrrolo[2,3-c]pyridine-5-carboxylates via the Pictet-Spengler Reaction. J. Org. Chem. 1998, 2731-2737. [CrossRef]

77. Nassar, M.N.; Nesarikar, V.N.; Lozano, R.; Parker, W.L.; Huang, Y.; Palaniswamy, V.; Xu, W.; Khaselev, N. Influence of formaldehyde impurity in polysorbate 80 and PEG-300 on the stability of a parenteral formulation of BMS-204352: Identification and control of the degradation product. Pharm. Dev. Technol. 2004, 9, 189-195. [CrossRef] [PubMed]

78. Bindra, D.S.; Williams, T.D.; Stella, V.J. Degradation of $\mathrm{O}^{6}$-Benzylguanine in Aqueous Polyethylene Glycol 400 (PEG 400) Solutions: Concerns with Formaldehyde in PEG 400. Pharm. Res. 1994, 11, 1060-1064. [CrossRef] [PubMed]

79. Hildebrandt, C.; Joos, L.; Saedler, R.; Winter, G. The "New Polyethylene Glycol Dilemma": Polyethylene Glycol Impurities and Their Paradox Role in mAb Crystallization. J. Pharm. Sci. 2015, 104, 1938-1945. [CrossRef] [PubMed]

80. Madhavi Sastry, G.; Adzhigirey, M.; Day, T.; Annabhimoju, R.; Sherman, W. Protein and ligand preparation: Parameters, protocols, and influence on virtual screening enrichments. J. Comput. Aided Mol. Des. 2013, 27, 221-234. [CrossRef] [PubMed]

81. Halgren, T.A.; Murphy, R.B.; Friesner, R.A.; Beard, H.S.; Frye, L.L.; Pollard, W.T.; Banks, J.L. Glide: A New Approach for Rapid, Accurate Docking and Scoring. 2. Enrichment Factors in Database Screening. J. Med. Chem. 2004, 47, 1750-1759. [CrossRef] [PubMed]

82. Friesner, R.A.; Murphy, R.B.; Repasky, M.P.; Frye, L.L.; Greenwood, J.R.; Halgren, T.A.; Sanschagrin, P.C.; Mainz, D.T. Extra Precision Glide: Docking and Scoring Incorporating a Model of Hydrophobic Enclosure for Protein-Ligand Complexes. J. Med. Chem. 2006, 49, 6177-6196. [CrossRef] [PubMed]

83. Friesner, R.A.; Banks, J.; Murphy, R.; Halgren, T.A.; Klicic, J.J.; Mainz, D.T.; Repasky, M.; Knoll, E.H.; Shelley, M.; Perry, J.; et al. Glide: A New Approach for Rapid, Accurate Docking and Scoring. 1. Method and Assessment of Docking Accuracy. J. Med. Chem. 2004, 47, 1739-1749. [CrossRef] [PubMed]

84. Delle Monache, G.; Misiti, D.; Salvatore, P.; Zappia, G.; Pierini, M. Stereoselective synthesis of 2-substituted 3-azabicyclo[3.2.0]heptan-2-ones by [2+2]-cycloaddition of $N$-allyl- $\beta$ - $N$-keteniminium salts derived from (R)-vinylglycinol. Tetrahedron Asymmetry 2000, 11, 2653-2659. [CrossRef]

85. Singh, P.; Panda, G. Intramolecular 5-endo-trig aminopalladation of $\beta$-hydroxy- $\gamma$-alkenylamine: Efficient route to a pyrrolidine ring and its application for the synthesis of (-)-8,8a-di-epi-swainsonine. RSC Adv. 2014, 4, 2161-2166. [CrossRef]

86. Wang, X.L.; Huang, W.F.; Lei, X.S.; Wei, B.G.; Lin, G.Q. A facile synthesis of 1,4-dideoxy-1,4-imino-L-ribitol (LRB) and (-)-8a-epi-swainsonine from D-glutamic acid. Tetrahedron 2011, 67, 4919-4923. [CrossRef]

87. Knippschild, U.; Milne, D.M.; Campbell, L.E.; DeMaggio, A.J.; Christenson, E.; Hoekstra, M.F.; Meek, D.W. p53 is phosphorylated in vitro and in vivo by the delta and epsilon isoforms of casein kinase 1 and enhances the level of casein kinase 1 delta in response to topoisomerase-directed drugs. Oncogene 1997, 15, 1727. [CrossRef] [PubMed]

88. Kabsch, W. Xds. Acta Crystallogr. Sect. D Biol. Crystallogr. 2010, 66, 125-132. [CrossRef] [PubMed]

89. Read, R.J. Pushing the boundaries of molecular replacement with maximum likelihood. Acta Crystallogr. Sect. D Biol. Crystallogr. 2001, 57, 1373-1382. [CrossRef]

90. Afonine, P.V.; Grosse-Kunstleve, R.W.; Echols, N.; Headd, J.J.; Moriarty, N.W.; Mustyakimov, M.; Terwilliger, T.C.; Urzhumtsev, A.; Zwart, P.H.; Adams, P.D. Towards automated crystallographic structure refinement with phenix.refine. Acta Crystallogr. Sect. D Biol. Crystallogr. 2012, 68, 352-367. [CrossRef] [PubMed] 
91. Emsley, P.; Lohkamp, B.; Scott, W.G.; Cowtan, K. Features and development of Coot. Acta Crystallogr. Sect. D Biol. Crystallogr. 2010, 66, 486-501. [CrossRef] [PubMed]

92. Moriarty, N.W.; Grosse-Kunstleve, R.W.; Adams, P.D. Electronic Ligand Builder and Optimization Workbench (eLBOW): A tool for ligand coordinate and restraint generation. Acta Crystallogr. Sect. D Biol. Crystallogr. 2009, 65, 1074-1080. [CrossRef] [PubMed]

Sample Availability: Samples of most compounds are available from A.L.

(C) 2019 by the authors. Licensee MDPI, Basel, Switzerland. This article is an open access article distributed under the terms and conditions of the Creative Commons Attribution (CC BY) license (http://creativecommons.org/licenses/by/4.0/). 